\title{
Evaluating the Accuracy of Firearm Examiner Conclusions using Cartridge Case Reproductions
}

\author{
Eric Freeman Law \\ West Virginia University, elaw2@mix.wvu.edu
}

Follow this and additional works at: https://researchrepository.wvu.edu/etd

Part of the Applied Statistics Commons, and the Other Physical Sciences and Mathematics Commons

\section{Recommended Citation}

Law, Eric Freeman, "Evaluating the Accuracy of Firearm Examiner Conclusions using Cartridge Case Reproductions" (2020). Graduate Theses, Dissertations, and Problem Reports. 7954.

https://researchrepository.wvu.edu/etd/7954

This Dissertation is protected by copyright and/or related rights. It has been brought to you by the The Research Repository @ WVU with permission from the rights-holder(s). You are free to use this Dissertation in any way that is permitted by the copyright and related rights legislation that applies to your use. For other uses you must obtain permission from the rights-holder(s) directly, unless additional rights are indicated by a Creative Commons license in the record and/ or on the work itself. This Dissertation has been accepted for inclusion in WVU Graduate Theses, Dissertations, and Problem Reports collection by an authorized administrator of The Research Repository @ WVU.

For more information, please contact researchrepository@mail.wvu.edu. 
Evaluating the Accuracy of Firearm Examiner Conclusions using Cartridge Case Reproductions

Eric Freeman Law

Follow this and additional works at: https://researchrepository.wvu.edu/etd

Part of the Applied Statistics Commons, and the Other Physical Sciences and Mathematics Commons 


\title{
Evaluating the Accuracy of Firearm Examiner Conclusions using Cartridge Case Reproductions
}

\author{
Eric Law \\ Dissertation submitted \\ to the Eberly College of Arts and Sciences \\ at West Virginia University
}

in partial fulfillment of the requirements for the degree of

Doctorate of Philosophy in

Forensic Science

Keith Morris, Ph.D., Chair

Jacqueline Speir, Ph.D.

Casper Venter, Ph.D.

Casey Jelsema, Ph.D.

Department of Forensic \& Investigative Science

\section{Morgantown, West Virginia \\ 2020}

Keywords: breech face, firing pin, error rates, double cast, cartridge case variability, congruent matching cells, conclusion variability, Bayesian network, likelihood ratio Copyright 2020 Eric Law 


\title{
ABSTRACT \\ Evaluating the Accuracy of Firearm Examiner Conclusions using Cartridge Case Reproductions
}

\author{
Eric Law
}

The forensic science pattern comparison areas, including fingerprints, footwear, and firearms, have been criticized for their subjective nature. While much research has attempted to move these disciplines to more objective methods, a majority of examiners are still coming to conclusions based on their own training and experience. To compare accuracy between examiners, a method called double-casting was used in this study to create plastic cartridge case reproductions. In the first part of this study, double-cast accuracy was evaluated using two automated comparison systems to quantify the similarity. It was determined that the double-casting method used here produces accurate reproductions with low variability between double-casts of the same master cartridge cases. In the second part of this study, 21 test sets were created to send to firearm examiners for comparison. Double-casts were created of the 21 test sets and mailed to each participant. The double-casts ensured that all participants were comparing exhibits with the same level of detail. Automated comparisons were then performed on the examiner test sets using the NIST toolmark comparison algorithms. The results showed that there are differences in the conclusions made by firearm examiners. Automated comparison systems were found to be complementary to examiners and should be used in combination. A Bayesian network was applied for further analysis of the examiner conclusion data based on likelihood ratios (LRs). Examiners were generally informative towards the true proposition in cartridge case comparisons, and inconclusive conclusions were found to provide evidential value with a LR approach. 


\section{Acknowledgements}

I would like to acknowledge:

- Dwight Deskins, a firearms examiner at the Kentucky State Police, for all of his assistance in refining the methodology for both casting and examiner comparisons.

- The firearms group at NIST for allowing access to the congruent matching cells (CMC) comparison algorithms, and for their assistance with automated comparisons throughout the duration of this project.

- The Sensofar group, including Cristina Cadevall and Adam Platteis, for their assistance when needed with 3D acquisitions and related software support.

- Adam Clark and Tom Tremmel from Productive Quality, Inc. (PQI) for their support when needed with the Sensofar system and 3D acquisitions. 


\section{Contents}

\begin{tabular}{lll}
\hline 1 & Preface & 1
\end{tabular}

2 The utility of double-casting for creating cartridge case $\begin{array}{ll}\text { reproductions } & 7\end{array}$

\begin{tabular}{|lll}
\hline 3 & Three-dimensional analysis of cartridge case double-casts & 34
\end{tabular}

$4 \quad$ Evaluating firearm examiner conclusion variability using $\begin{array}{ll}\text { cartridge case reproductions } & 54\end{array}$

5 Bayesian network for the analysis of firearm examiner $\begin{array}{ll}\text { conclusions } & 83\end{array}$

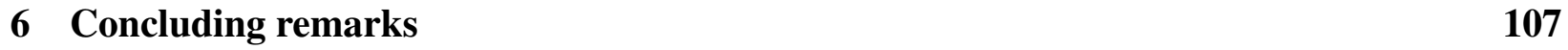

\begin{tabular}{|lll}
\hline 7 & Supplementary material & 111
\end{tabular} 


\section{Preface}

Firearm examiners are often tasked with comparing cartridge cases recovered from a crime scene to cartridge cases test fired from a suspect firearm. Traditionally, a visual comparison is conducted using a comparison microscope. The crime scene cartridge cases are compared to each of the test fires, and any observed similarities and differences are noted. An examiner then reports a conclusion regarding whether the crime scene and test fired cartridge cases were fired by the same firearm.

The National Academy of Sciences (NAS) report noted that firearms examination lacks a precisely defined process [1]. The Association of Firearm and Toolmark Examiners (AFTE) provides the most current recommendations for firearm examiners to follow, and their guidelines are based on "sufficient agreement" between two sets of marks in determining whether they were made by the same tool (firearm) [2]. The concept of "sufficient agreement" is not well defined and causes examiners to draw on their own training and experience. Furthermore, the AFTE guidelines do not address reliability and reproducibility of comparisons because limited studies exist where these concepts have been studied. Similarly, the President's Council of Advisors on Science and Technology (PCAST) noted that to establish the foundational validity of the firearms examination discipline, it must be repeatable, reproducible, and accurate [3].

Previous research has assessed examiner accuracy in comparisons. Some studies have used consecutively manufactured firearms [4-7]. These are considered a worst case scenario because consecutively manufactured surfaces should show the closest non-match similarity. The studies by Bunch and Murphy [4] and LaPorte [6] resulted in no false positive or false negative conclusions. The study performed by Lyons [5] had a 1.7\% error rate, and the Fadul study [7] had an average examiner error rate of less than $0.1 \%$. Another group of research utilized firearms that are commonly encountered in casework [8-11]. The studies by Smith [8] and Stroman [10] showed no false positives or false negatives. The study by Baldwin et al. [9] resulted in a false positive rate of $1.01 \%$ and a false negative rate of $0.367 \%$. Lastly, the study done by Smith et al. [11] showed a sensitivity of $91.4 \%$ and a specificity of $90.5 \%$ for cartridge case comparisons. The previously discussed literature has established low rates of error in the firearms examination discipline.

Less research has focused on the reproducibility aspect of foundational validity because of complications due to shot-to-shot variability within the reproduction of markings on cartridge cases 
fired by the same firearm. A cartridge is made up of four components that may have an effect on the impressions left by a firearm: the cartridge case, primer, powder or propellant, and bullet (Figure 1.1). When the firing pin of a firearm strikes the primer of the cartridge, the propellant is ignited and burns leading to an increase in pressure within the cartridge. The bullet is then forced out of the cartridge case and down the barrel of the firearm. The cartridge case is extracted from the chamber and subsequently ejected, either automatically from a semi-automatic pistol or rifle, or manually from a revolver or bolt action rifle. The firing pin strike leaves the firing pin impression, and the buildup in pressure due to the burning propellant forces the cartridge case rearward against the breech face of the firearm resulting in the breech face impression. An extractor and ejector mark are also present, however, they are less useful for identification. If a cartridge case has been reloaded, multiple extractor and ejector marks may be present making it difficult to determine which mark came from the most recent shot.

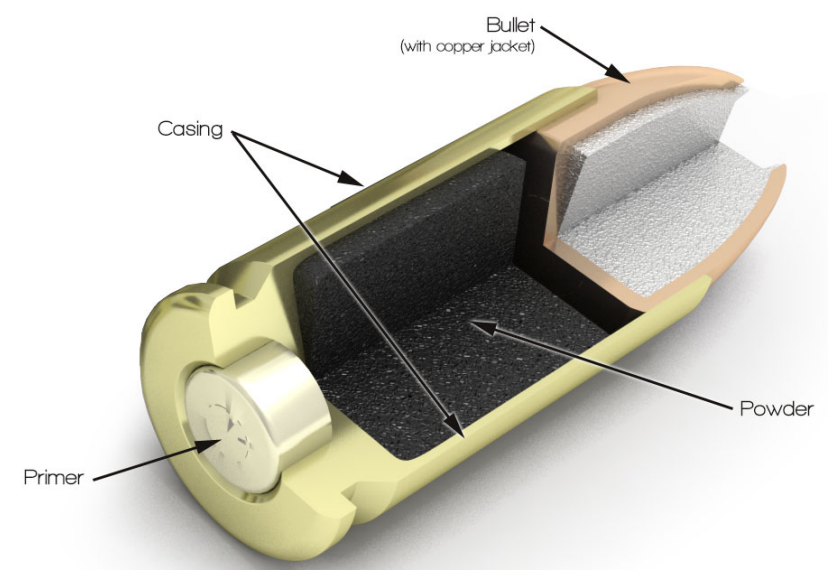

Figure 1.1: The four components making up a cartridge [12].

The brand and volume of propellant used will have an effect on the pressure inside the chamber of the firearm and therefore the force with which the cartridge case is pushed against the breech face [13]. Because the primer is where the breech face and firing pin impressions occur, the composition of the primer will have an effect on the impressions left based on the malleability of the material [14]. Due to these factors, cartridge cases fired from a single firearm will have variability within the reproduced markings. Figure 1.2 illustrates this effect. Both of the cartridge cases shown were fired from the same Taurus 24/7 G2 pistol chambered in $9 \mathrm{~mm}$ Luger using the same Federal American Eagle ammunition. They were collected from a set of ten consecutive shots. Although the exact number of shots between the two cartridge cases is unknown, this poses a challenge for examiners. While similarities are present, there are also some differences. Notably, there is significant flowback around the firing pin impression in Figure 1.2 (left) that is not present in Figure 1.2 (right). In a comparison accuracy study, these two cartridge cases may each represent 
a questioned cartridge case sent to each of two examiners. Due to the differences in marking reproduction, the difficulty level for each examiner may not be the same. This prevents a direct comparison between the conclusions of each examiner even though the cartridge cases were fired by the same firearm. However, it is important to analyze examiner conclusions using the same evidence because the comparison process relies on subjective methods leading to the possibility of examiners coming to different conclusions given the same comparisons.
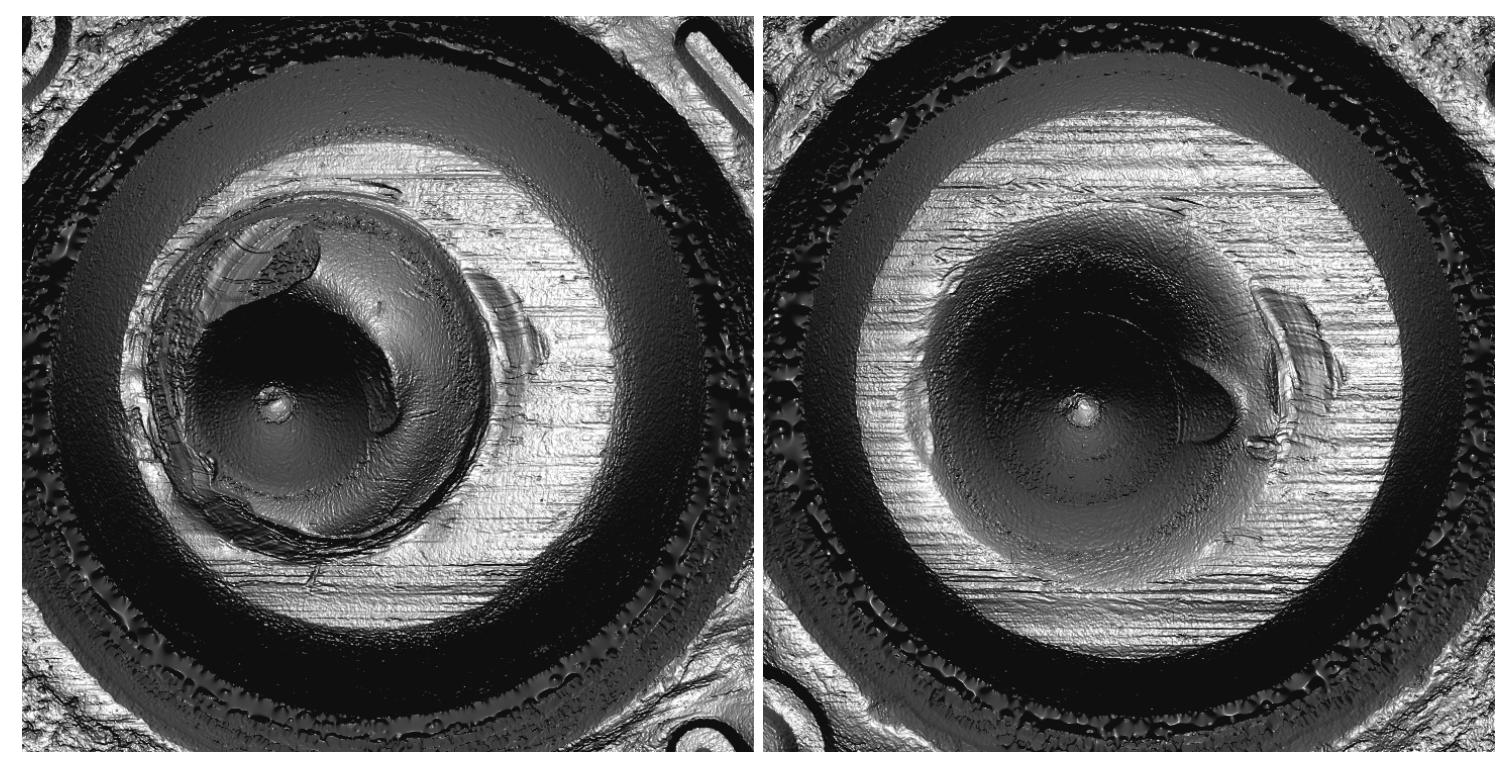

Figure 1.2: Cartridge case variability example. Both cartridge cases were fired from the same Taurus 24/7 G2 pistol. Images saved using the Cadre X3P Viewer [15].

To compare examiner conclusions given the same evidence, digital images of comparison sets can be shared with participants. A study by Duez et al. [16] utilized three-dimensional (3D) scans of cartridge cases and virtual comparison microscopy (VCM). There were no false positives or false negatives made by trained examiners, however, one trainee made two false positives. Another study by Mattijssen et al. [17] analyzed conclusion variability by presenting examiners with twodimensional (2D) comparison images of Glock cartridge case aperture shear. A false positive rate of $19.0 \%$ and false negative rate of $6.8 \%$ were found. The authors noted that the high false positive rate was likely do to the selection of difficult comparisons. In both of the above studies, examiners were generally found to reach the same conclusions given the same evidence. One limitation to using digital representations of cartridge cases is that participants are restricted to using only those areas captured during imaging. To conduct a conclusion variability study using physical samples, a method of reproduction is needed.

A method called double-casting has been used for creating cartridge case reproductions [18]. Using this method, a silicone mold is produced of a master cartridge case, creating an impression 
negative. A liquid plastic mix is then poured into the silicone mold. Once cured, the double-cast is a plastic reproduction of the markings from the master cartridge case. A proficiency test has been conducted by the European Network of Forensic Science Institutes (ENFSI) using doublecasts [19]. The cartridge case comparisons resulted in 15 false positive conclusions and seven false negative conclusions. Fourteen of the false positives were on a single set, and the cause was determined to be due to significant subclass characteristic effects.

Accuracy of the reproduced markings and variability within the double-casting method has yet to be objectively demonstrated. This is critical if test sets are to be replicated numerous times. Once the double-casting method has been verified to produce accurate reproductions and low variability within and between different molds of the same master cartridge cases, double-casts can be used for visual comparisons and database imaging. Double-casts were applied in this study for analyzing examiner conclusion variability using physical comparison sets. Chapters 2 and 3 of this paper focus on analysis of the double-casting method utilizing 2D and 3D automated comparison systems, respectively. Chapters 4 and 5 discuss the results of the examiner study that were based on comparisons performed using double-casts. 


\section{References}

[1] National Academy of Sciences. Strengthening Forensic Science in the United States: A Path Forward. The National Academies Press, 2009.

[2] Virginia Department of Forensic Science. Firearm/Toolmark Procedures Manual, 2017.

[3] President's Council of Advisors on Science and Technology. Forensic Science in Criminal Courts: Ensuring Scientific Validity of Feature-Comparison Methods, 2016.

[4] Stephen G. Bunch and Douglas P. Murphy. A comprehensive validity study for the forensic examination of cartridge cases. AFTE Journal, 35(2):201-203, 2003.

[5] Dennis J. Lyons. The identification of consecutively manufactured extractors. AFTE Journal, 41(3):246-256, 2009.

[6] Dawn LaPorte. An empirical and validation study of breechface marks on .380 ACP caliber cartridge cases fired from ten consecutively finished Hi-Point C9 pistols. AFTE Journal, 43(4):303-309, 2011.

[7] Thomas G. Fadul Jr, Gabriel A. Hernandez, Stephanie Stoiloff, and Sneh Gulati. An empirical study to improve the scientific foundation of forensic firearm and tool mark identification utilizing 10 consecutively manufactured slides. AFTE Journal, 45(4):376-393, 2013.

[8] Erich D. Smith. Cartridge case and bullet comparison validation study with firearms submitted in casework. AFTE Journal, 36(4):130-135, 2004.

[9] David P. Baldwin, Stanley J. Bajic, Max Morris, and Daniel Zamzow. A study of false-positive and false-negative error rates in cartridge case comparisons. Retrieved from https://www.ncjrs.gov/pdffiles1/nij/249874.pdf, 2014.

[10] Angela Stroman. Empirically determined frequency of error in cartridge case examinations using a declared double-blind format. AFTE Journal, 46(2):157-175, 2014.

[11] Tasha P. Smith, G. Andrew Smith, and Jeffrey B. Snipes. A validation study of bullet and cartridge case comparisons using samples representative of actual casework. Journal of Forensic Sciences, 61(4):939-946, 2016.

[12] Guns \& Ammo 101. The bullet cartridge. Retrieved from http://www.gunsandammo.info/ammo/ammo-101, 2015. 
[13] Otto Mezger, Walter Heess, Fritz Hasslacher, and Robert Heindl. Determination of the type of pistol employed from an examination of fired bullets and shells. The American Journal of Police Science, 2(6):473-499, 1931.

[14] Jan De Kinder, Frederic Tulleners, and Hugues Thiebaut. Reference ballistic imaging database performance. Forensic Science International, 140:207-215, 2004.

[15] Cadre Forensics. X3P Viewer Software. Available by request at www.cadreforensics.com as of 21-Feb-2020.

[16] Pierre Duez, Todd Weller, Marcus Brubaker, Richard E. Hockensmith, and Ryan Lilien. Development and validation of a virtual examination tool for firearm forensics. Journal of Forensic Sciences, 63(4):1069-1084, 2018.

[17] Erwin J.A.T. Mattijssen, Cilia L.M. Witteman, Charles E.H. Berger, Nicolaas W. Brand, and Reinoud D. Stoel. Validity and reliability of forensic firearm examiners. Forensic Science International, 307, 2020.

[18] INTERPOL. INTERPOL Ballistics Information Network: Handbook on the Collection and Sharing of Ballistics Data, 3rd edition, 2014.

[19] Petra Pauw-Vugts, Alice Walters, Leif Øren, Kripos, and Leopold Pfoser. Faid2009:

Proficiency test and workshop. AFTE Journal, 45(2):115-127, 2013. 


\title{
2. The utility of double-casting for creating cartridge case reproductions
}

\section{Publication status}

This chapter appears as a standalone publication in the Association of Firearm and Toolmark Examiners (AFTE) Journal. Citation: Eric Law and Keith Morris. The utility of double-casting for creating cartridge case reproductions. AFTE Journal, 52(1):26-39, 2020.

\begin{abstract}
Due to the variability that arises in the breech face and firing pin impressions on multiple cartridge cases fired by the same firearm, a method of reproduction is needed to create standardized cartridge cases. A process called double-casting has been used that involves creating a silicone rubber mold of a cartridge case, and then uses a plastic resin casting material to make a cartridge case reproduction. The first part of this study analyzed casts of the National Institute of Standards and Technology (NIST) standard cartridge case (SRM 2461). Integrated Ballistic Identification System (IBIS ${ }^{\circledR}$ ) data and visual comparisons showed the method of double-casting was able to reproduce fine detail that is visible even at $100 \times$ magnification. Prior to widespread use, it is important to characterize the effects of within- and between-mold variability of the casts. Cartridge cases fired by three firearms were replicated using the double-casting process and entered into an IBIS $^{\circledR}$. Some instances of significant differences between the mold and cast sets were found. However, visual comparisons of 1) the casts to their original cartridge cases, and 2) the casts to other casts of the same cartridge case both showed that the double-casting method reliably reproduces the fine detail that is present on the original cartridge cases.
\end{abstract}




\section{Introduction}

When a firearm examiner test fires a firearm in the laboratory for known exemplars to perform a cartridge case comparison, there may be significant variability within the breech face and firing pin impressions on the test fired cartridge cases. Many factors influence this variability, such as ammunition type (e.g. manufacturer, primer, and powder) and the firearm used. An example of this can be seen in Figure 1, where two Federal ${ }^{\circledR}$ American Eagle ${ }^{\circ} 9 \mathrm{~mm}$ Luger cartridge cases were collected from the same SCCY ${ }^{\circledR}$ CPX-2 pistol that exhibit dissimilarities, including a firing pin drag mark present on one cartridge case but not the other. To develop standard cartridge cases to use for training, proficiency testing, and quality control of automated databases, a method of high-quality reproduction is necessary to ensure consistent impression quality and uniformity across all copies produced, thereby avoiding the significant variability in cycle-of-fire marks that can frequently be found across multiple cartridge cases fired by the same firearm, if they were to be used as test specimens.

The National Institute of Standards and Technology (NIST) maintains a Standard Reference Material (SRM) 2460 and 2461 standard bullets and cartridge cases project (the focus in this study will be on the standard cartridge case SRM 2461) [1] [2]. The NIST standard cartridge cases are created from a master cartridge case that is reproduced using electroforming [3]. A master specimen is placed into a container with electrolytes producing a negative impression on the master specimen. The negative is then immersed in a conductive solution and through the electroforming process metal is deposited onto the negative, creating a positive that is the reproduced material. For SRM 2461, a fired master cartridge case is reproduced through electroforming with nickel and brass and then mounted onto a brass cylinder to resemble an actual fired cartridge case [1]. 

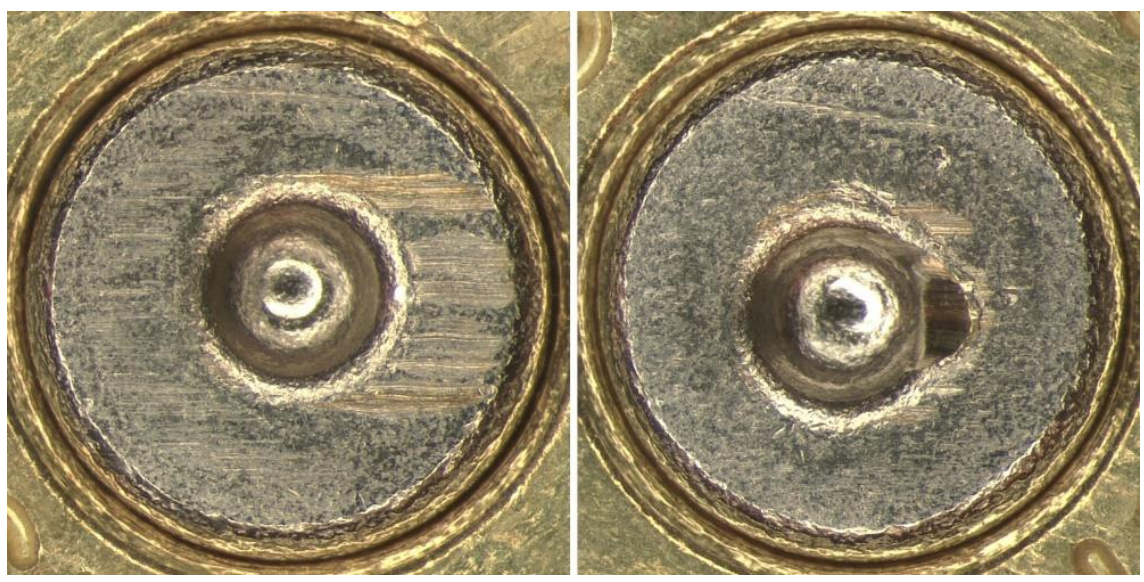

Figure 1: Primers of two fired $9 \mathrm{~mm}$ Luger cartridge cases from the same SCCY® CPX-2 at 20× magnification. Both were illuminated using fluorescent ring lights on a Leica® MZ75 stereomicroscope and imaged with a Ludesco® Resolve HD camera.

A somewhat more efficient way to create cartridge case reproductions is through doublecasting, where a silicone mold is created of fired cartridge cases and then plastic resin casts are made using the mold [4]. Double-casting does not produce replicas that have the appearance of metal as the NIST standard cartridge cases created through electroforming; however, it is a simple method for producing high-quality plastic reproductions. Double-casting allows forensic laboratories to easily produce their own reference materials from evidence cartridge cases.

Prior to widespread use of double-casting, it is important to characterize both within- and between-mold variability. Ideally, all molds of a particular cartridge case, and all casts from that mold, will have the same markings. However, there is likely variability within both the molding and casting processes that may affect the fine detail visible on the cartridge case reproductions. The purpose of this research was to analyze variability in the casting process by using an automated database and visual comparisons of casts from multiple firearms.

\section{Materials and Methods}

\section{Double-Casting Method}

Prior to the mold and cast variability study, the method used to create the molds and casts must first be described. The molds were created by covering a cartridge case with Smooth-On® Mold Star ${ }^{\mathrm{TM}}$ 30, a two-part liquid silicone rubber mix, and allowing it to cure in a pressure pot for six hours at room temperature. The pressure pot was a Grizzly® 2 1/2 gallon paint tank with all the 
valves closed; Teflon ${ }^{\mathrm{TM}}$ thread sealant was applied to any openings where pressure could leak, such as any screw threads. The purpose for this was to allow the pot to hold constant pressure to force air bubbles out of the liquid as the silicone cured. Once the mold had cured and the cartridge case was removed, Smooth-On® Smooth Cast ${ }^{\circledR}$ 327, a two-part plastic resin mix, was dyed black with Smooth-On® SO-Strong ${ }^{\circledR}$ black colorant and poured into the mold. The plastic resin was allowed to cure for six hours in the pressure pot at room temperature. Once the plastic had cured, it was removed from the mold, creating the cartridge case cast that was a reproduction of the original fired cartridge case (the master cartridge case). A mold here is defined as a single negative impression of a single fired cartridge case.

\section{NIST Standard Cartridge Case}

As an initial study to provide some data on how well the casts reproduce the detail present on a master cartridge case, the NIST standard cartridge case was chosen because it is currently the only standard cartridge case available to be used by forensic laboratories [1]. West Virginia University has five of the NIST SRM 2461 standard cartridge cases with identifiers 2P2333, 2P2335, 2P2415, 2P4316, and 2P6325 that are all reproductions of a single master cartridge case using electroforming.

Standard cartridge case 2P2335 was randomly selected for molding and casting. One mold and six casts from that mold were created. These six casts were entered into an Integrated Ballistic Identification System $^{\circledR}$ (IBIS) Heritage ${ }^{\mathrm{TM}}$ System for automated image comparison. In a previous study [5], the five NIST standards were entered ten times each by three users, so there were 150 images of all five standards in the database for the casts to be compared against. The IBIS ${ }^{\circledR}$ data were extracted into Microsoft ${ }^{\circledR}$ Excel® spreadsheets for analysis

Comparison microscopy was also used to compare the NIST standard cartridge case to the casts created from it to analyze the level of detail that was reproduced using the casting method detailed above. All images were taken using a Leica ${ }^{\circledR}$ FS C comparison microscope and a Nikon® D5200 camera.

\section{Double Cast Variability}

Three $9 \mathrm{~mm}$ Luger pistols were selected for the analysis of variability within and between molds: a SCCY ${ }^{\circledR}$ CPX-2, a Hi-Point ${ }^{\circledR}$ C9, and a Smith \& Wesson ${ }^{\circledR}$ SD9VETM. These specific 
pistols were selected for the variety of markings they leave on cartridge cases, including firing pin aperture shearing, parallel impressed breech face marks, and granular breech face impression patterns. Twenty-five cartridge cases were fired in each of these three firearms. The cartridge cases were collected, engraved with a letter and number, and cleaned in a sonic cleaner using Hornady ${ }^{\circledR}$ One Shot ${ }^{\circledR}$ Sonic Clean solution designed for cartridge cases.

One mold was created of each of the 25 cartridge cases fired by each of the three firearms for a total of 75 individual cartridge case molds. As previously defined, a mold is a single negative impression of the master cartridge case. For each cartridge case mold, five casts were made, for a total of 125 casts per firearm for each of the three firearms (a total of 375 casts). For compactness, the molding process used can incorporate 25 negative impressions of a cartridge case in a circular block of silicone that is approximately $11.5 \mathrm{~cm}$ in diameter and $4.0 \mathrm{~cm}$ thick. Updating the definition of a mold, there were now three molds total that each contained 25 negative impressions of the cartridge cases from each firearm. This process was repeated two more times, for a total of three molds per firearm, each of which contained 25 negative impressions. A total of five sets of casts were created from each of the three molds for each firearm for a total of 1,125 cartridge case reproductions. The casts were all labeled and stored in a way that allowed for every individual cast to be identified back to the specific mold from which it originated so that both within- and betweenmold variability were able to be analyzed. Figure 2 displays a visual representation of this process for casts of Cartridge Case \#1 for a single firearm. Each cast labeled with a "1" is a reproduction of the same master cartridge case. Similarly, there would be 15 casts labeled with a " 2 ", 15 labeled with a "3", and so on up to number 25. 


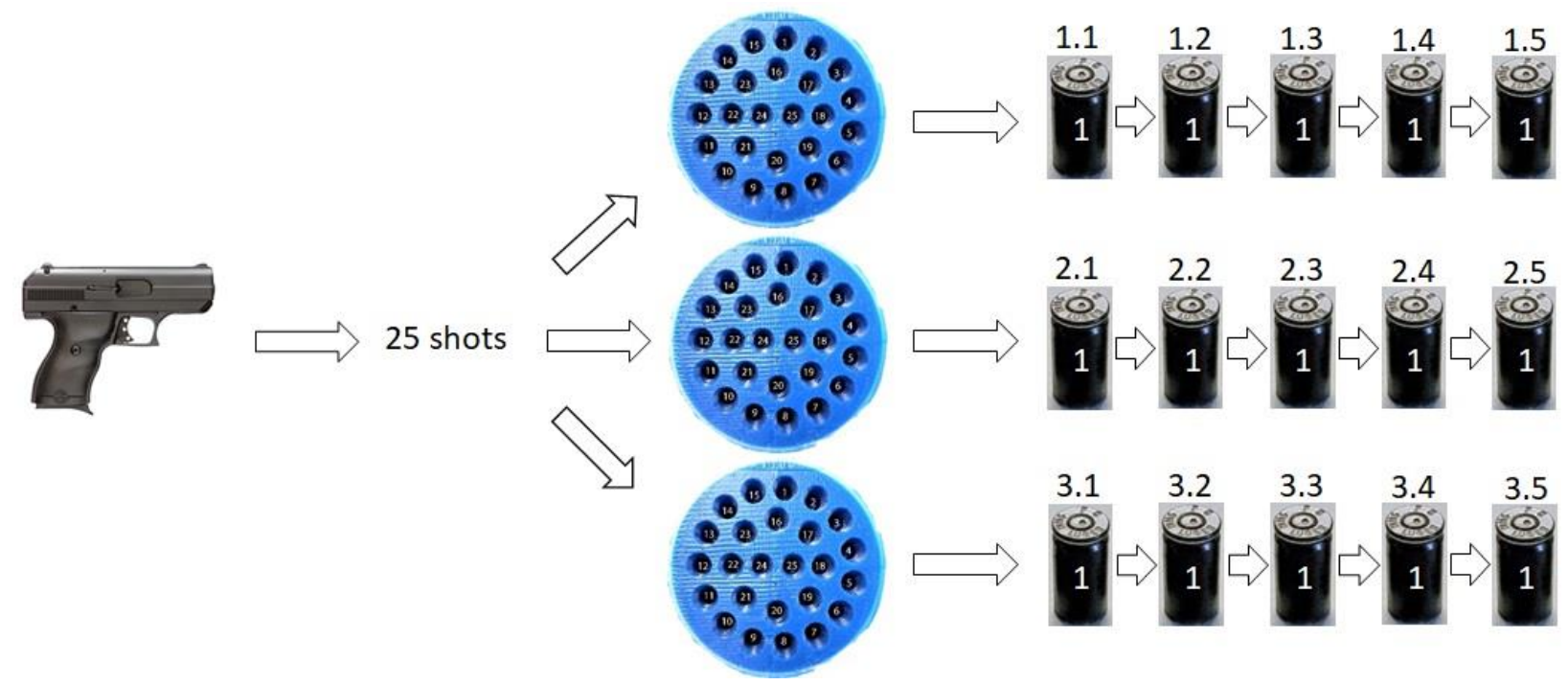

Figure 2: Representation of the mold process for a single firearm. The numbers above each cast represent the mold and set numbers. For example, 1.4 corresponds to the first mold and fourth set of casts created from that mold. This was repeated for the other two firearms in the study.

The master cartridge cases and casts were all entered into an IBIS $®$ Heritage ${ }^{\mathrm{TM}}$ System for automated correlation. Because the IBIS ${ }^{\circledR}$ compares in a pairwise fashion, after all cartridge cases and casts were entered the correlation requests were deleted and re-run for the purpose of comparing every exhibit to all others. The correlation data were extracted into Microsoft ${ }^{\circledR}$ Excel ${ }^{\circledR}$ spreadsheets. For plotting and statistical analysis, the spreadsheets for this part of the study were set up as 15 column by 25 row matrices of IBIS ${ }^{\circledR}$ scores (refer to Table 1 as an example). The columns corresponded to the mold and set number, and the rows corresponded to the 25 master cartridge cases. In Table 1, the cells correspond to the similarity scores where, for example, 1.2.4 corresponds to the first mold, the second set of casts from that mold, and the reproduction of master cartridge case number four. Therefore, six spreadsheets were created, one for the breech face and firing pin scores for each of the three firearms. Throughout this study, the breech face and firing pin scores were used as a simple similarity metric for the cartridge case reproductions. 
Table 1: Example data table of the IBIS score results.

\begin{tabular}{|c|c|c|c|c|c|c|}
\hline Original Cartridge Case & $\begin{array}{l}\text { Mold 1/Set } 1 \\
\text { Similarity }\end{array}$ & $\begin{array}{l}\text { Mold 1/Set 2 } \\
\text { Similarity }\end{array}$ & $\cdots$ & $\begin{array}{ll}\text { Mold 2/Set } 3 \\
\text { Similarity }\end{array}$ & ... & $\begin{array}{l}\text { Mold 3/Set 5 } \\
\text { Similarity }\end{array}$ \\
\hline 1 & 1.1 .1 & 1.2 .1 & $\ldots$ & 2.3 .1 & $\ldots$ & 3.5 .1 \\
\hline 2 & 1.1 .2 & 1.2 .2 & $\ldots$ & 2.3 .2 & $\ldots$ & 3.5 .2 \\
\hline 3 & 1.1 .3 & 1.2 .3 & $\ldots$ & 2.3 .3 & $\ldots$ & 3.5 .3 \\
\hline 4 & 1.1 .4 & 1.2 .4 & $\ldots$ & 2.3 .4 & $\ldots$ & 3.5 .4 \\
\hline 5 & 1.1 .5 & 1.2 .5 & $\cdots$ & 2.3 .5 & $\cdots$ & 3.5 .5 \\
\hline : & : & : & $:$ & : & : & : \\
\hline 25 & 1.1 .25 & 1.2 .25 & $\ldots$ & 2.3 .25 & $\ldots$ & 3.5 .25 \\
\hline
\end{tabular}

To evaluate the IBIS ${ }^{\circledR}$ scores of the cast sets to determine if there were any statistically significant differences, the Friedman test was used. This test was selected because the cast sets are all related to each other (i.e. there are several casts of the same master cartridge cases in the data set). The Friedman test involves ranking the data within each row in a matrix of values, rather than within each column, and allows for comparing several related samples because it does not make any assumption of independence between the cast sets [6]. Statistical results were evaluated at the 0.05 level of significance. The Friedman test is a global test, so a significant $p$-value (less than 0.05) indicates that there are significant differences somewhere within the data set. When the Friedman test is significant, a multiple comparisons procedure can be performed on the data ranks to determine which groups are different, as detailed by Conover and Iman [7]. The Friedman test and multiple comparisons procedures were carried out using the PMCMRplus R package [8] [9].

To complement the similarity scores from the IBIS ${ }^{\circledR}$, visual comparisons were performed with a Leica® FS C comparison microscope. Images were taken with a Nikon® D5200 camera to analyze the reproduction of fine detail between 1) the casts and master cartridge cases, 2) casts of the same cartridge case created from the same mold but different sets from that mold, and 3) casts of the same cartridge case created from the different molds.

\section{Results and Discussion}

NIST Standard Cartridge Case

Low magnification images $(20 \times)$ were taken of the NIST standard cartridge case and its cast to illustrate an overall view of the level of detail reproduced on the primer area during casting (Figure 3). A 100× magnification comparison image is shown in Figure 4 for visualization of the 
fine detail on the master cartridge case and the cast. Visually, the detail from the master cartridge case appears to be reproduced well on to the cast. Lighting the casts can be a challenge due to them being a black plastic that is not as reflective as an actual cartridge case. However, this can also make them better for imaging because of the different reflective properties of the plastic.
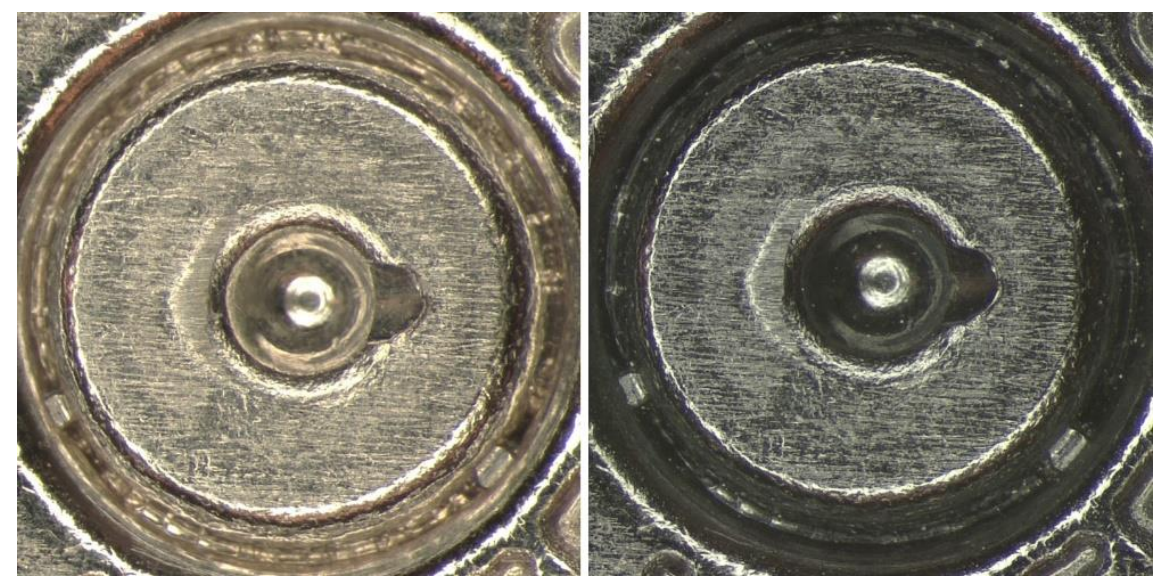

Figure 3: Images of the NIST standard cartridge case (left) and its cast (right) at 20× magnification. Both were illuminated using fluorescent ring lights on a Leica MZ75 stereomicroscope and imaged with a Ludesco Resolve HD camera.
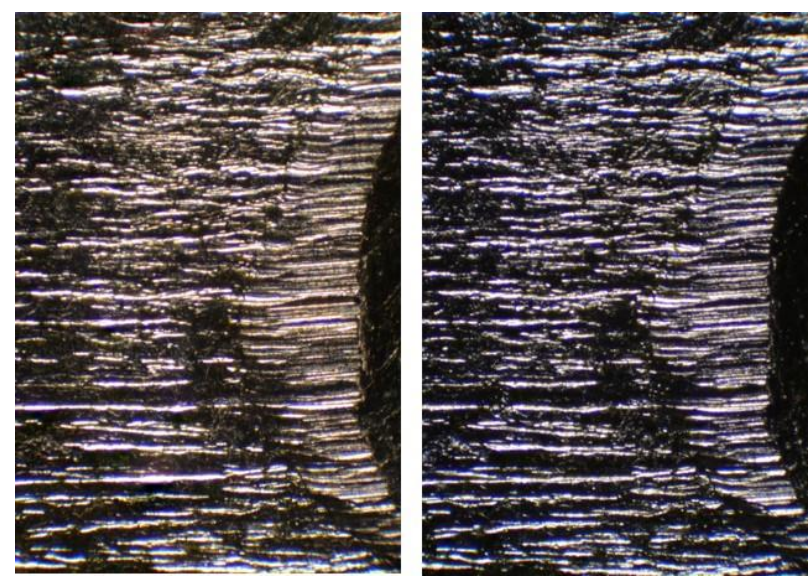

Figure 4: Images of the NIST standard cartridge case (left) and its cast (right) at 100× magnification. Both were illuminated using fiber optic oblique lighting on a Leica ${ }^{\circledR}$ FS C comparison microscope and imaged with a Nikon ${ }^{\circledR}$ D5200 camera.

Each of the six casts was entered into the IBIS ${ }^{\circledR}$ to compare against the master NIST standard cartridge cases (2P2333, 2P2335, 2P2415, 2P4316, and 2P6325). The results for the sixth 
cast are displayed in Figure 5. Because the IBIS ${ }^{\circledR}$ compares exhibits in a pairwise manner, the sixth cast was correlated against the five casts entered first, and these other casts are represented in Figure 5 by circles with crosshairs. These cast-to-cast comparisons outlined by the dashed box are grouped at the highest similarity scores based on both breech face and firing pin impressions. The data outlined by the solid box in Figure 5 represent the cast of 2P2335 compared to the five NIST standard cartridge cases. The filled circles within the solid box are the comparisons of the cast of 2P2335 to the actual 2P2335 NIST standard cartridge case. The open circles are the comparisons of the cast of 2P2335 to the other four actual NIST standard cartridge cases (2P2333, 2P2415, 2P4316, and 2P6325). Recall, the IBIS ${ }^{\circledR}$ database contained 150 total images of the five NIST standard cartridge cases from a previous study [5]. These cast-to-master cartridge case comparisons are all grouped slightly below the cast-to-cast comparisons, but both groups show complete separation from the non-matches (the " $X$ " in Figure 4) when considering breech face and firing pin scores jointly.

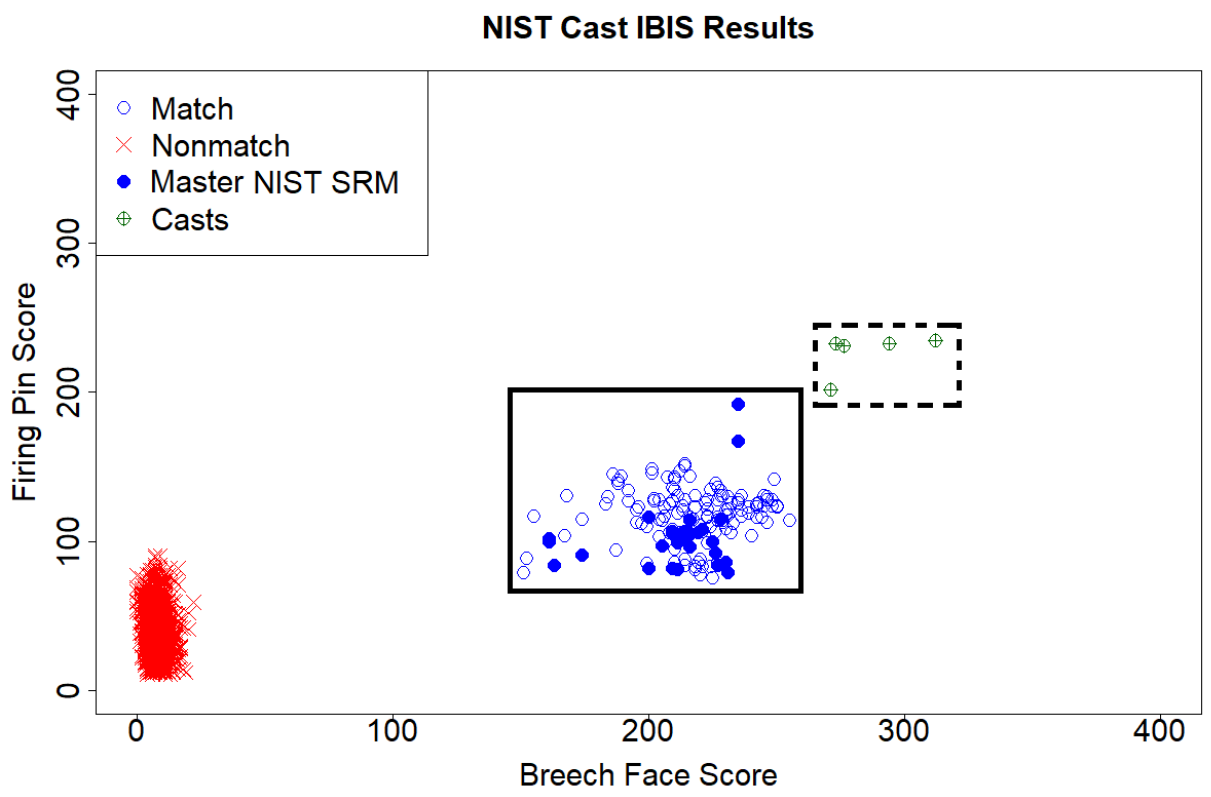

Figure 5: Plot of the match and non-match IBIS® results of the NIST cast data. 


\section{Double Cast Variability IBIS® Data}

The IBIS ${ }^{\circledR}$ results are displayed in Figures 6, 7, and 8. The boxplots show a panel for both the breech face and firing pin with the cast set numbers on the x-axis and IBIS ${ }^{\circledR}$ score on the $y$ axis. For example, Cast Set 2.3 refers to the second mold and the third set of casts created from that mold. The IBIS ${ }^{\circledR}$ scores correspond to the comparison of the casts to the master cartridge cases from which they were molded. This score was expected to be high because it was hypothesized that double-casting would produce excellent reproductions. Within- and between-mold variability is illustrated in these plots. It appears that there is a decreasing trend in the IBIS ${ }^{\circledR}$ scores because the scores decrease as the mold number increases (1.x vs. 2.x vs. 3.x). This is especially apparent when comparing all of the sets created from the first mold to the sets created from the second and third molds.

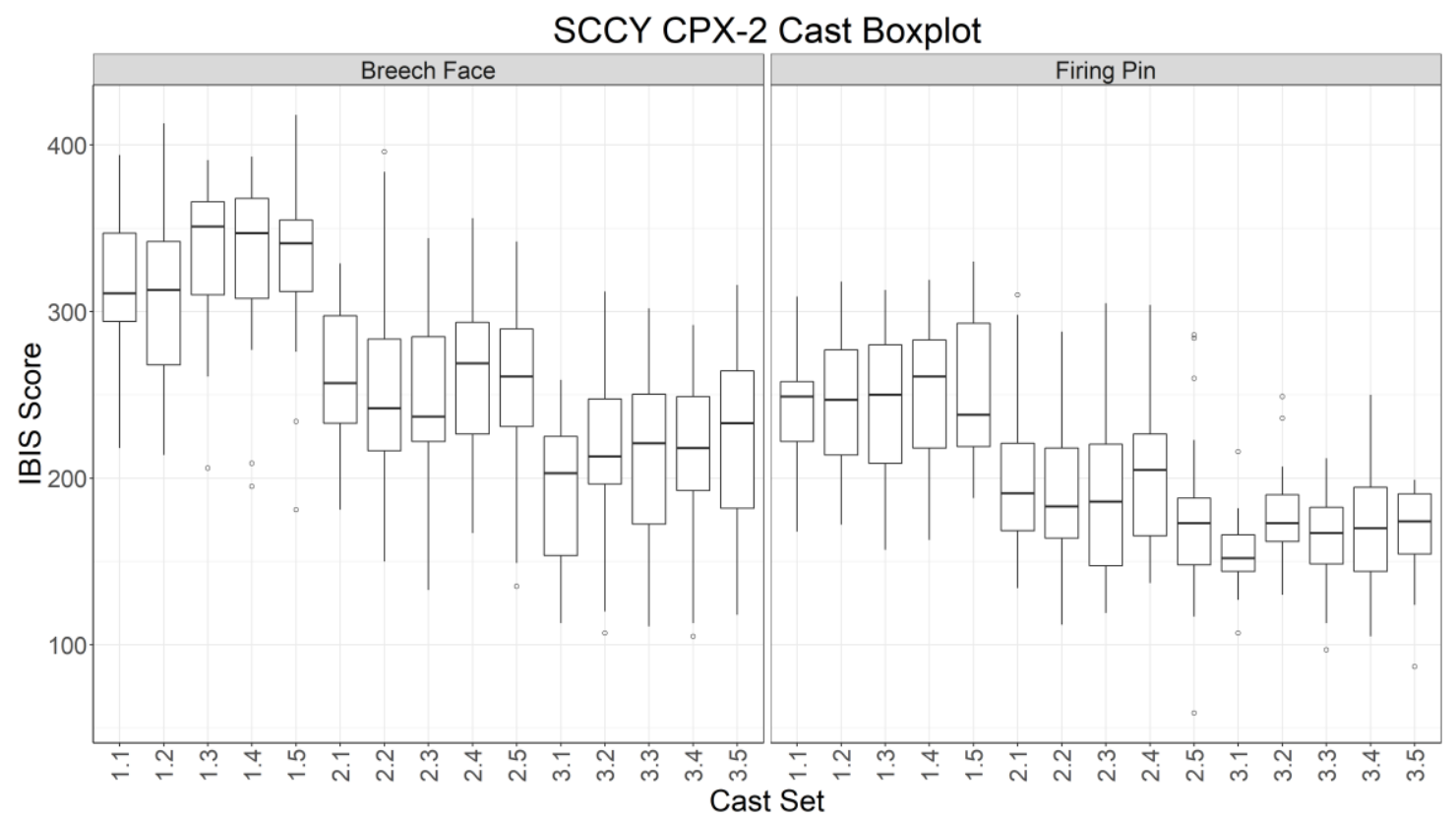

Figure 6: Boxplot of the $S C C Y ® C P X-2$ casts compared to their master cartridge cases. 


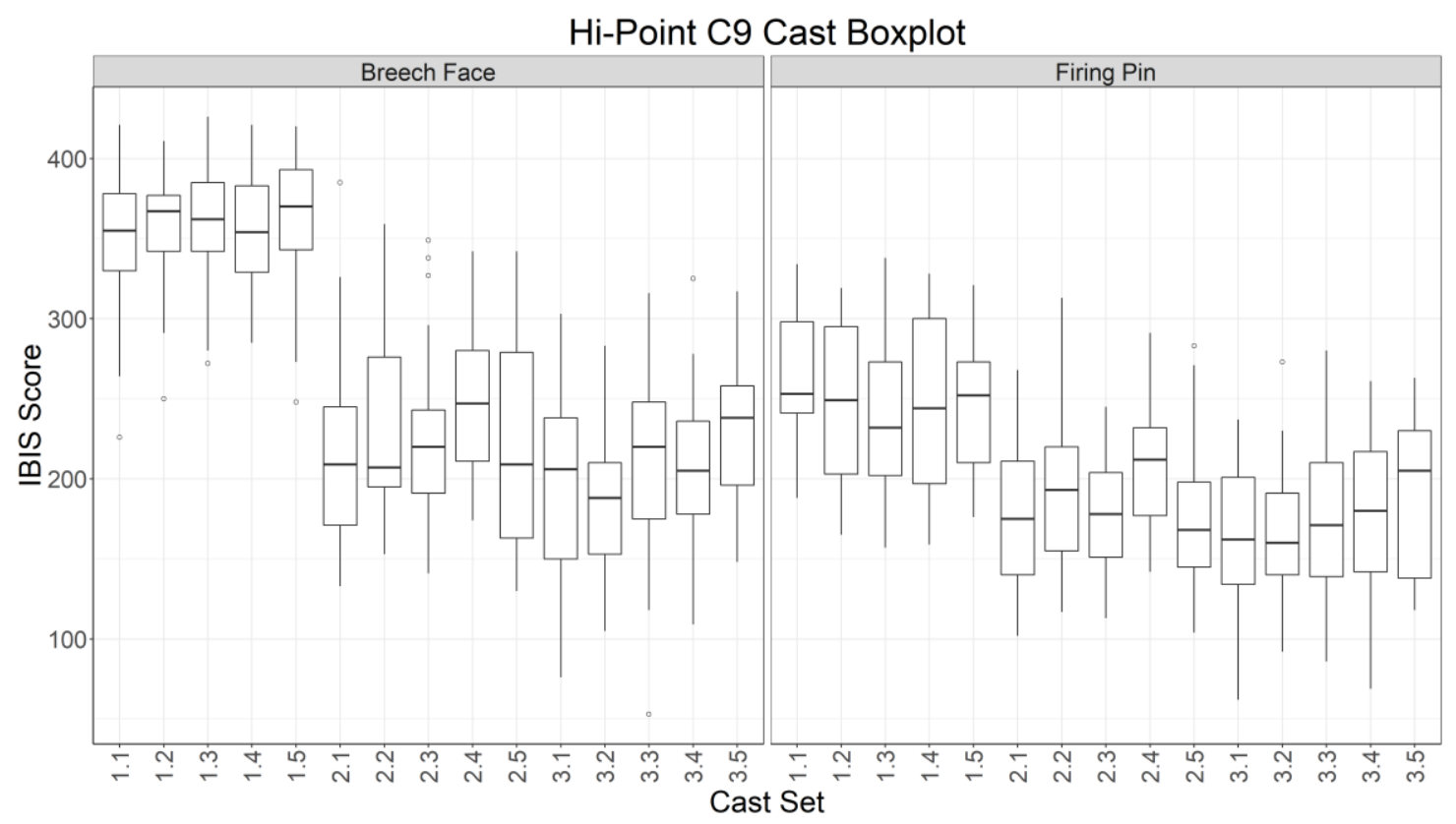

Figure 7: Boxplot of the Hi-Point ${ }^{\circledR}$ C9 casts compared to their master cartridge cases.

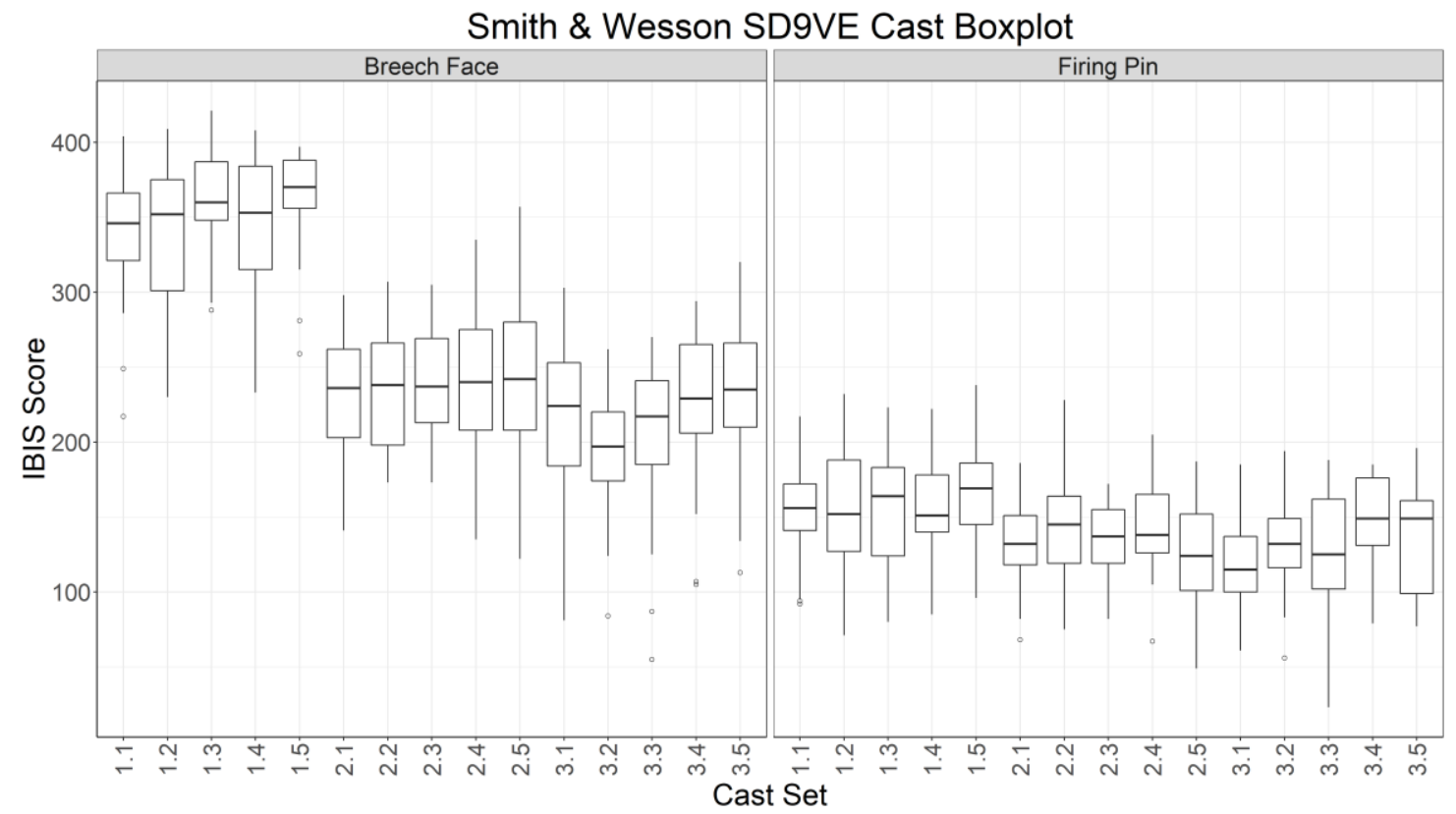

Figure 8: Boxplot of the Smith \& Wesson ${ }^{\circledR} S D 9 V E^{\mathrm{TM}}$ casts compared to their master cartridge cases.

To evaluate the cast-to-master cartridge case scores, the Friedman test was used. In all instances, the Friedman test was significant at the 0.05 level. A multiple comparisons procedure was then performed, and the resulting $p$-values are displayed in Tables 2 through 7. Recall, the 
data were arranged as 15 column by 25 row matrices, where the columns were the mold/set numbers and the rows were cartridge cases 1 through 25 fired by each firearm. The data were ranked across each row from 1 to 15 , where rank one was the largest IBIS ${ }^{\circledR}$ score and rank 15 was the smallest. The columns and rows in Tables 2 through 7 are the pairwise comparisons of the columns (mold/set numbers) from the matrices generated from the IBIS ${ }^{\circledR}$ data. All instances of statistical significance at the 0.05 level are highlighted in gray. Overall, only $3.6 \%$ of the differences in sets from all guns were from comparisons that did not include Mold 1. All other differences were when Mold 1 sets were compared to Mold 2 and Mold 3 sets. Only one instance of a significant difference within-mold was found, and this was between Set 4 and Set 5 from Mold 2 of the Hi-Point ${ }^{\circledR} \mathrm{C} 9$ when comparing firing pin scores. All other differences were from betweenmold sets.

Table 2: SCCY® CPX-2 breech face Friedman test multiple comparison p-values. The global test p-value was less than 0.0001 .

\begin{tabular}{|c|c|c|c|c|c|c|c|c|c|c|c|c|c|c|}
\hline \multicolumn{15}{|c|}{ SCCY CPX-2 Breech Face } \\
\hline & 1.1 & 1.2 & 1.3 & 1.4 & 1.5 & 2.1 & 2.2 & 2.3 & 2.4 & 2.5 & 3.1 & 3.2 & 3.3 & 3.4 \\
\hline 1.2 & 1.0000 & - & - & - & - & - & - & - & - & - & - & - & - & - \\
\hline 1.3 & 1.0000 & 0.9941 & - & - & - & - & - & - & - & - & - & - & - & - \\
\hline 1.4 & 1.0000 & 1.0000 & 1.0000 & - & - & - & - & - & - & - & - & - & - & - \\
\hline 1.5 & 1.0000 & 0.9997 & 1.0000 & 1.0000 & - & - & - & - & - & - & - & - & - & - \\
\hline 2.1 & 0.1197 & 0.3751 & 0.0087 & 0.0592 & 0.0284 & - & - & - & - & - & - & - & - & - \\
\hline 2.2 & 0.0170 & 0.0872 & 0.0007 & 0.0068 & 0.0027 & 1.0000 & - & - & - & - & - & - & - & - \\
\hline 2.3 & 0.0093 & 0.0536 & 0.0003 & 0.0035 & 0.0013 & 1.0000 & 1.0000 & - & - & - & - & - & - & - \\
\hline 2.4 & 0.3013 & 0.6627 & 0.0353 & 0.1744 & 0.0956 & 1.0000 & 0.9996 & 0.9980 & - & - & - & - & - & - \\
\hline 2.5 & 0.0191 & 0.0956 & 0.0008 & 0.0077 & 0.0031 & 1.0000 & 1.0000 & 1.0000 & 0.9997 & - & - & - & - & - \\
\hline 3.1 & $<0.0001$ & $<0.0001$ & $<0.0001$ & $<0.0001$ & $<0.0001$ & 0.0134 & 0.1001 & 0.1544 & 0.0029 & 0.0913 & - & - & - & - \\
\hline 3.2 & $<0.0001$ & 0.0002 & $<0.0001$ & $<0.0001$ & $<0.0001$ & 0.6509 & 0.9583 & 0.9836 & 0.3641 & 0.9509 & 0.9678 & - & - & - \\
\hline 3.3 & $<0.0001$ & 0.0001 & $<0.0001$ & $<0.0001$ & $<0.0001$ & 0.5044 & 0.8995 & 0.9509 & 0.2452 & 0.8861 & 0.9905 & 1.0000 & - & - \\
\hline 3.4 & $<0.0001$ & 0.0001 & $<0.0001$ & $<0.0001$ & $<0.0001$ & 0.6147 & 0.9469 & 0.9778 & 0.3320 & 0.9381 & 0.9756 & 1.0000 & 1.0000 & - \\
\hline 3.5 & $<0.0001$ & 0.0004 & $<0.0001$ & $<0.0001$ & $<0.0001$ & 0.7838 & 0.9867 & 0.9960 & 0.5044 & 0.9836 & 0.9176 & 1.0000 & 1.0000 & 1.0000 \\
\hline
\end{tabular}


Table 3: SCCY@ CPX-2 firing pin Friedman test multiple comparison p-values. The global test pvalue was less than 0.0001 .

\begin{tabular}{|c|c|c|c|c|c|c|c|c|c|c|c|c|c|c|}
\hline \multicolumn{15}{|c|}{ SCCY CPX-2 Firing Pin } \\
\hline & 1.1 & 1.2 & 1.3 & 1.4 & 1.5 & 2.1 & 2.2 & 2.3 & 2.4 & 2.5 & 3.1 & 3.2 & 3.3 & 3.4 \\
\hline 1.2 & 1.0000 & - & - & - & - & - & - & - & - & - & - & - & - & - \\
\hline 1.3 & 1.0000 & 1.0000 & - & - & - & - & - & - & - & - & - & - & - & - \\
\hline 1.4 & 1.0000 & 1.0000 & 1.0000 & - & - & - & - & - & - & - & - & - & - & - \\
\hline 1.5 & 1.0000 & 0.9999 & 0.9991 & 1.0000 & - & - & - & - & - & - & - & - & - & - \\
\hline 2.1 & 0.2273 & 0.2444 & 0.3854 & 0.1955 & 0.0201 & - & - & - & - & - & - & - & - & - \\
\hline 2.2 & 0.0390 & 0.0433 & 0.0868 & 0.0314 & 0.0016 & 1.0000 & - & - & - & - & - & - & - & - \\
\hline 2.3 & 0.0125 & 0.0141 & 0.0314 & 0.0098 & 0.0004 & 0.9998 & 1.0000 & - & - & - & - & - & - & - \\
\hline 2.4 & 0.6854 & 0.7083 & 0.8474 & 0.6381 & 0.1538 & 1.0000 & 0.9941 & 0.9545 & - & - & - & - & - & - \\
\hline 2.5 & 0.0006 & 0.0007 & 0.0020 & 0.0005 & $<0.0001$ & 0.9424 & 0.9995 & 1.0000 & 0.5649 & - & - & - & - & - \\
\hline 3.1 & $<0.0001$ & $<0.0001$ & $<0.0001$ & $<0.0001$ & $<0.0001$ & 0.0252 & 0.1669 & 0.3416 & 0.0019 & 0.8390 & - & - & - & - \\
\hline 3.2 & 0.0003 & 0.0003 & 0.0009 & 0.0002 & $<0.0001$ & 0.8711 & 0.9965 & 0.9999 & 0.4197 & 1.0000 & 0.9228 & - & - & - \\
\hline 3.3 & $<0.0001$ & $<0.0001$ & $<0.0001$ & $<0.0001$ & $<0.0001$ & 0.3523 & 0.8027 & 0.9424 & 0.0683 & 0.9998 & 0.9997 & 1.0000 & - & - \\
\hline 3.4 & $<0.0001$ & $<0.0001$ & 0.0001 & $<0.0001$ & $<0.0001$ & 0.5772 & 0.9379 & 0.9904 & 0.1603 & 1.0000 & 0.9948 & 1.0000 & 1.0000 & - \\
\hline 3.5 & $<0.0001$ & $<0.0001$ & 0.0001 & $<0.0001$ & $<0.0001$ & 0.6139 & 0.9507 & 0.9933 & 0.1808 & 1.0000 & 0.9924 & 1.0000 & 1.0000 & 1.0000 \\
\hline
\end{tabular}

Table 4: Hi-Point ${ }^{\circledR}$ C9 breech face Friedman test multiple comparison p-values. The global test p-value was less than 0.0001 .

\begin{tabular}{|c|c|c|c|c|c|c|c|c|c|c|c|c|c|c|}
\hline \multicolumn{15}{|c|}{ Hi-Point C9 Breech Face } \\
\hline & 1.1 & 1.2 & 1.3 & 1.4 & 1.5 & 2.1 & 2.2 & 2.3 & 2.4 & 2.5 & 3.1 & 3.2 & 3.3 & 3.4 \\
\hline 1.2 & 1.0000 & - & - & - & - & - & - & - & - & - & - & - & - & - \\
\hline 1.3 & 1.0000 & 1.0000 & - & - & - & - & - & - & - & - & - & - & - & - \\
\hline 1.4 & 1.0000 & 1.0000 & 1.0000 & - & - & - & - & - & - & - & - & - & - & - \\
\hline 1.5 & 1.0000 & 1.0000 & 1.0000 & 1.0000 & - & - & - & - & - & - & - & - & - & - \\
\hline 2.1 & $<0.0001$ & $<0.0001$ & $<0.0001$ & $<0.0001$ & $<0.0001$ & - & - & - & - & - & - & - & - & - \\
\hline 2.2 & 0.0020 & 0.0004 & 0.0005 & 0.0005 & 0.0001 & 0.9980 & - & - & - & - & - & - & - & - \\
\hline 2.3 & 0.0013 & 0.0003 & 0.0003 & 0.0003 & 0.0001 & 0.9992 & 1.0000 & - & - & - & - & - & - & - \\
\hline 2.4 & 0.0408 & 0.0123 & 0.0139 & 0.0147 & 0.0032 & 0.8216 & 1.0000 & 0.9999 & - & - & - & - & - & - \\
\hline 2.5 & $<0.0001$ & $<0.0001$ & $<0.0001$ & $<0.0001$ & $<0.0001$ & 1.0000 & 0.9999 & 1.0000 & 0.9393 & - & - & - & - & - \\
\hline 3.1 & $<0.0001$ & $<0.0001$ & $<0.0001$ & $<0.0001$ & $<0.0001$ & 0.9989 & 0.6118 & 0.6806 & 0.1292 & 0.9887 & - & - & - & - \\
\hline 3.2 & $<0.0001$ & $<0.0001$ & $<0.0001$ & $<0.0001$ & $<0.0001$ & 0.9794 & 0.3394 & 0.4030 & 0.0430 & 0.9145 & 1.0000 & - & - & - \\
\hline 3.3 & $<0.0001$ & $<0.0001$ & $<0.0001$ & $<0.0001$ & $<0.0001$ & 1.0000 & 0.9677 & 0.9813 & 0.5529 & 1.0000 & 1.0000 & 0.9991 & - & - \\
\hline 3.4 & $<0.0001$ & $<0.0001$ & $<0.0001$ & $<0.0001$ & $<0.0001$ & 1.0000 & 0.9145 & 0.9436 & 0.4030 & 0.9999 & 1.0000 & 0.9999 & 1.0000 & - \\
\hline 3.5 & 0.0003 & 0.0000 & 0.0001 & 0.0001 & 0.0000 & 1.0000 & 1.0000 & 1.0000 & 0.9961 & 1.0000 & 0.8903 & 0.6694 & 0.9989 & 0.9936 \\
\hline
\end{tabular}


Table 5: Hi-Point ${ }^{\circledR}$ C9 firing pin Friedman test multiple comparison p-values. The global test pvalue was less than 0.0001 .

\begin{tabular}{|c|c|c|c|c|c|c|c|c|c|c|c|c|c|c|}
\hline \multicolumn{15}{|c|}{ Hi-Point C9 Firing Pin } \\
\hline & 1.1 & 1.2 & 1.3 & 1.4 & 1.5 & 2.1 & 2.2 & 2.3 & 2.4 & 2.5 & 3.1 & 3.2 & 3.3 & 3.4 \\
\hline 1.2 & 0.9993 & - & - & - & - & - & - & - & - & - & - & - & - & - \\
\hline 1.3 & 0.9728 & 1.0000 & - & - & - & - & - & - & - & - & - & - & - & - \\
\hline 1.4 & 0.9955 & 1.0000 & 1.0000 & - & - & - & - & - & - & - & - & - & - & - \\
\hline 1.5 & 0.9998 & 1.0000 & 1.0000 & 1.0000 & - & - & - & - & - & - & - & - & - & - \\
\hline 2.1 & $<0.0001$ & $<0.0001$ & 0.0001 & $<0.0001$ & $<0.0001$ & - & - & - & - & - & - & - & - & - \\
\hline 2.2 & 0.0001 & 0.0103 & 0.0523 & 0.0217 & 0.0068 & 0.9703 & - & - & - & - & - & - & - & - \\
\hline 2.3 & $<0.0001$ & 0.0001 & 0.0007 & 0.0002 & $<0.0001$ & 1.0000 & 0.9987 & - & - & - & - & - & - & - \\
\hline 2.4 & 0.0916 & 0.7134 & 0.9435 & 0.8384 & 0.6348 & 0.0764 & 0.9300 & 0.2293 & - & - & - & - & - & - \\
\hline 2.5 & $<0.0001$ & $<0.0001$ & $<0.0001$ & $<0.0001$ & $<0.0001$ & 1.0000 & 0.9087 & 1.0000 & 0.0368 & - & - & - & - & - \\
\hline 3.1 & $<0.0001$ & $<0.0001$ & $<0.0001$ & $<0.0001$ & $<0.0001$ & 1.0000 & 0.5409 & 0.9961 & 0.0041 & 1.0000 & - & - & - & - \\
\hline 3.2 & $<0.0001$ & $<0.0001$ & $<0.0001$ & $<0.0001$ & $<0.0001$ & 0.9997 & 0.4250 & 0.9875 & 0.0022 & 1.0000 & 1.0000 & - & - & - \\
\hline 3.3 & $<0.0001$ & $<0.0001$ & $<0.0001$ & $<0.0001$ & $<0.0001$ & 1.0000 & 0.8621 & 1.0000 & 0.0255 & 1.0000 & 1.0000 & 1.0000 & - & - \\
\hline 3.4 & $<0.0001$ & 0.0002 & 0.0020 & 0.0006 & 0.0001 & 1.0000 & 0.9999 & 1.0000 & 0.3810 & 0.9999 & 0.9773 & 0.9476 & 0.9996 & - \\
\hline 3.5 & 0.0003 & 0.0229 & 0.1000 & 0.0451 & 0.0155 & 0.9144 & 1.0000 & 0.9919 & 0.9773 & 0.8036 & 0.3810 & 0.2811 & 0.7347 & 0.9991 \\
\hline
\end{tabular}

Table 6: Smith \& Wesson ${ }^{\circledR}$ SD9VE ${ }^{\mathrm{TM}}$ breech face Friedman test multiple comparison p-values. The global test p-value was less than 0.0001 .

\begin{tabular}{|c|c|c|c|c|c|c|c|c|c|c|c|c|c|c|}
\hline \multicolumn{15}{|c|}{ Smith \& Wesson SD9VE Breech Face } \\
\hline & 1.1 & 1.2 & 1.3 & 1.4 & 1.5 & 2.1 & 2.2 & 2.3 & 2.4 & 2.5 & 3.1 & 3.2 & 3.3 & 3.4 \\
\hline 1.2 & 1.0000 & - & - & - & - & - & - & - & - & - & - & - & - & - \\
\hline 1.3 & 0.9996 & 1.0000 & - & - & - & - & - & - & - & - & - & - & - & - \\
\hline 1.4 & 1.0000 & 1.0000 & 1.0000 & - & - & - & - & - & - & - & - & - & - & - \\
\hline 1.5 & 0.9813 & 0.9993 & 1.0000 & 0.9993 & - & - & - & - & - & - & - & - & - & - \\
\hline 2.1 & $<0.0001$ & $<0.0001$ & $<0.0001$ & $<0.0001$ & $<0.0001$ & - & - & - & - & - & - & - & - & - \\
\hline 2.2 & 0.0053 & 0.0010 & 0.0001 & 0.0010 & $<0.0001$ & 0.9980 & - & - & - & - & - & - & - & - \\
\hline 2.3 & 0.0036 & 0.0006 & $<0.0001$ & 0.0006 & $<0.0001$ & 0.9992 & 1.0000 & - & - & - & - & - & - & - \\
\hline 2.4 & 0.0013 & 0.0002 & $<0.0001$ & 0.0002 & $<0.0001$ & 1.0000 & 1.0000 & 1.0000 & - & - & - & - & - & - \\
\hline 2.5 & 0.0077 & 0.0014 & 0.0001 & 0.0014 & $<0.0001$ & 0.9956 & 1.0000 & 1.0000 & 1.0000 & - & - & - & - & - \\
\hline 3.1 & $<0.0001$ & $<0.0001$ & $<0.0001$ & $<0.0001$ & $<0.0001$ & 1.0000 & 0.9974 & 0.9989 & 0.9999 & 0.9943 & - & - & - & - \\
\hline 3.2 & $<0.0001$ & $<0.0001$ & $<0.0001$ & $<0.0001$ & $<0.0001$ & 0.9677 & 0.2906 & 0.3497 & 0.5293 & 0.2377 & 0.9728 & - & - & - \\
\hline 3.3 & $<0.0001$ & $<0.0001$ & $<0.0001$ & $<0.0001$ & $<0.0001$ & 1.0000 & 0.9348 & 0.9585 & 0.9909 & 0.9029 & 1.0000 & 0.9995 & - & - \\
\hline 3.4 & 0.0001 & $<0.0001$ & $<0.0001$ & $<0.0001$ & $<0.0001$ & 1.0000 & 1.0000 & 1.0000 & 1.0000 & 0.9998 & 1.0000 & 0.8622 & 0.9999 & - \\
\hline 3.5 & 0.0008 & 0.0001 & 0.0000 & 0.0001 & $<0.0001$ & 1.0000 & 1.0000 & 1.0000 & 1.0000 & 1.0000 & 1.0000 & 0.6118 & 0.9961 & 1.0000 \\
\hline
\end{tabular}


Table 7: Smith \& Wesson ${ }^{\circledR} S D 9 V E^{\mathrm{TM}}$ firing pin Friedman test multiple comparison p-values. The global test p-value was less than 0.0001 .

\begin{tabular}{|c|c|c|c|c|c|c|c|c|c|c|c|c|c|c|}
\hline \multicolumn{15}{|c|}{ Smith \& Wesson SD9VE Firing Pin } \\
\hline & 1.1 & 1.2 & 1.3 & 1.4 & 1.5 & 2.1 & 2.2 & 2.3 & 2.4 & 2.5 & 3.1 & 3.2 & 3.3 & 3.4 \\
\hline 1.2 & 1.0000 & - & - & - & - & - & - & - & - & - & - & - & - & - \\
\hline 1.3 & 1.0000 & 1.0000 & - & - & - & - & - & - & - & - & - & - & - & - \\
\hline 1.4 & 1.0000 & 1.0000 & 1.0000 & - & - & - & - & - & - & - & - & - & - & - \\
\hline 1.5 & 0.9898 & 0.9898 & 0.9874 & 0.9999 & - & - & - & - & - & - & - & - & - & - \\
\hline 2.1 & 0.2448 & 0.2448 & 0.2620 & 0.0759 & 0.0028 & - & - & - & - & - & - & - & - & - \\
\hline 2.2 & 0.9886 & 0.9886 & 0.9909 & 0.8830 & 0.2709 & 0.9860 & - & - & - & - & - & - & - & - \\
\hline 2.3 & 0.4125 & 0.4125 & 0.4349 & 0.1571 & 0.0081 & 1.0000 & 0.9983 & - & - & - & - & - & - & - \\
\hline 2.4 & 0.9548 & 0.9548 & 0.9615 & 0.7543 & 0.1571 & 0.9977 & 1.0000 & 0.9999 & - & - & - & - & - & - \\
\hline 2.5 & 0.0384 & 0.0384 & 0.0425 & 0.0076 & 0.0001 & 1.0000 & 0.7441 & 0.9998 & 0.8760 & - & - & - & - & - \\
\hline 3.1 & 0.0009 & 0.0009 & 0.0010 & 0.0001 & $<0.0001$ & 0.9511 & 0.1337 & 0.8537 & 0.2364 & 0.9998 & - & - & - & - \\
\hline 3.2 & 0.0660 & 0.0660 & 0.0725 & 0.0145 & 0.0003 & 1.0000 & 0.8458 & 1.0000 & 0.9389 & 1.0000 & 0.9985 & - & - & - \\
\hline 3.3 & 0.1699 & 0.1699 & 0.1833 & 0.0470 & 0.0014 & 1.0000 & 0.9645 & 1.0000 & 0.9918 & 1.0000 & 0.9792 & 1.0000 & - & - \\
\hline 3.4 & 0.9973 & 0.9973 & 0.9980 & 0.9473 & 0.3905 & 0.9582 & 1.0000 & 0.9918 & 1.0000 & 0.6103 & 0.0795 & 0.7337 & 0.9139 & - \\
\hline 3.5 & 0.8537 & 0.8537 & 0.8688 & 0.5514 & 0.0725 & 0.9999 & 1.0000 & 1.0000 & 1.0000 & 0.9645 & 0.4125 & 0.9874 & 0.9993 & 1.0000 \\
\hline
\end{tabular}

To understand how significant differences were determined, a line plot was created and is shown in Figure 9. The example here is from the Smith \& Wesson ${ }^{\circledR}$ SD9VE ${ }^{\mathrm{TM}}$ breech face scores. The bold line follows all casts of master Cartridge Case \#1 out of 25, and displays the variability in scores through all five sets from each of the three molds. This line plot can be directly related to the boxplots and the results from the Friedman test multiple comparisons procedure. For example, Sets 1.5 and 2.1 were found to be significantly different (Table 6), and in the line plot there is a sharp drop in score for all of the casts. The statistical significance here is due to how the Friedman test multiple comparisons procedure works. Similar to the line in the line plot, the change in rank across the sets for each cartridge case is being considered. Statistical significance depends on the increase or decrease in rank, and therefore score, of each cast from one set to the next. Currently, Figure 9 shows the sequential comparisons of all the sets. To compare Set 1.1 and Set 3.5, imagine a line directly connecting each cast from Set 1.1 to each cast from Set 3.5. A similar exercise could be used to compare any two groups of interest. 


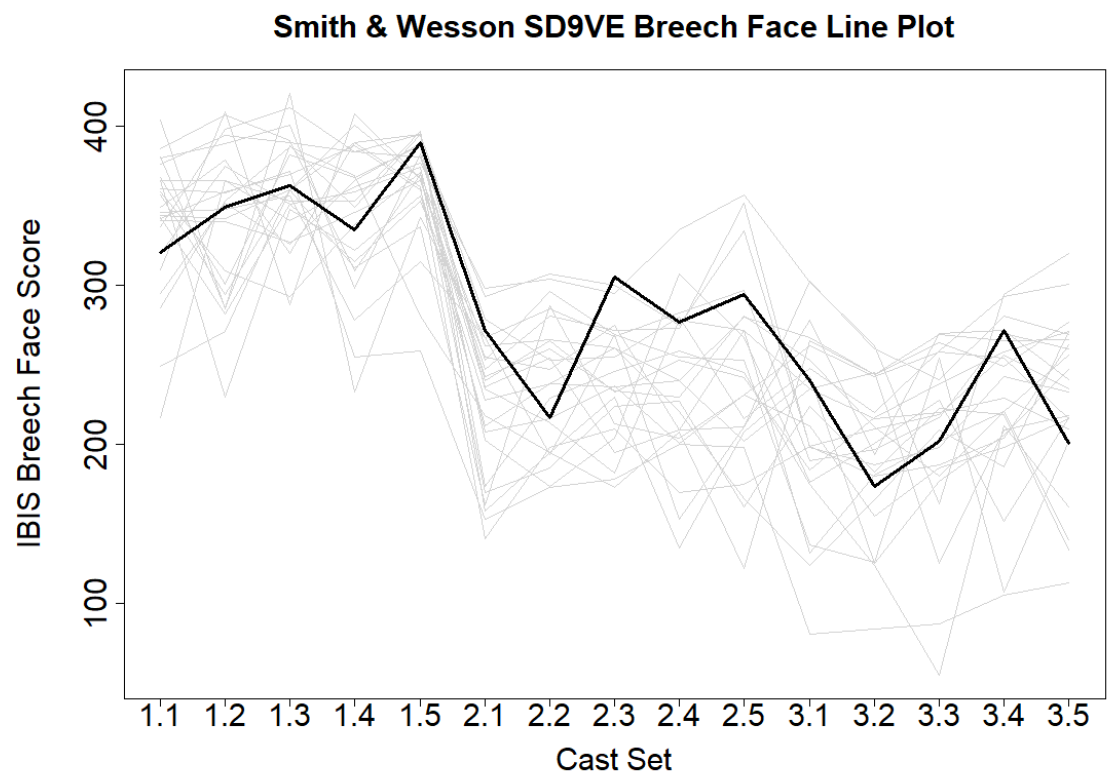

Figure 9: Line plot for the comparison of cast sets from the Smith \& Wesson ${ }^{\circledR} S D 9 V E^{\mathrm{TM}}$ breech face scores.

All previous comparisons have been between the casts and their master cartridge cases. Figure 10 shows the IBIS ${ }^{\circledR}$ scores of the cast-to-cast comparisons for all casts of each master cartridge case compared to each other. For example, the scores of all casts of Cartridge Case \#1 compared to each other are shown, as well as all the casts of Cartridge Case \#2 compared to each other, and so on. No comparisons of Cartridge Case \#1 casts to Cartridge Case \#2 casts are included, nor any similar comparison up to Cartridge Case \#25. The SCCY® CPX-2 is shown as an example, but the other two firearms showed the same trends. Composing the match data are within-mold comparisons displayed by the different shapes and colors and between-mold comparisons displayed in black. No actual cartridge cases are included in the match data, but both actual cartridge cases and casts are included in the non-matching data. The within-mold comparison scores appear to be grouped at higher IBIS ${ }^{\circledR}$ scores than between-mold comparisons, although they do overlap with each other. However, there is no clear visual separation of Mold 1, Mold 2, and Mold 3 comparison scores. Perfect separation from the non-matches was achieved when considering both breech face and firing pin correlation scores together. 


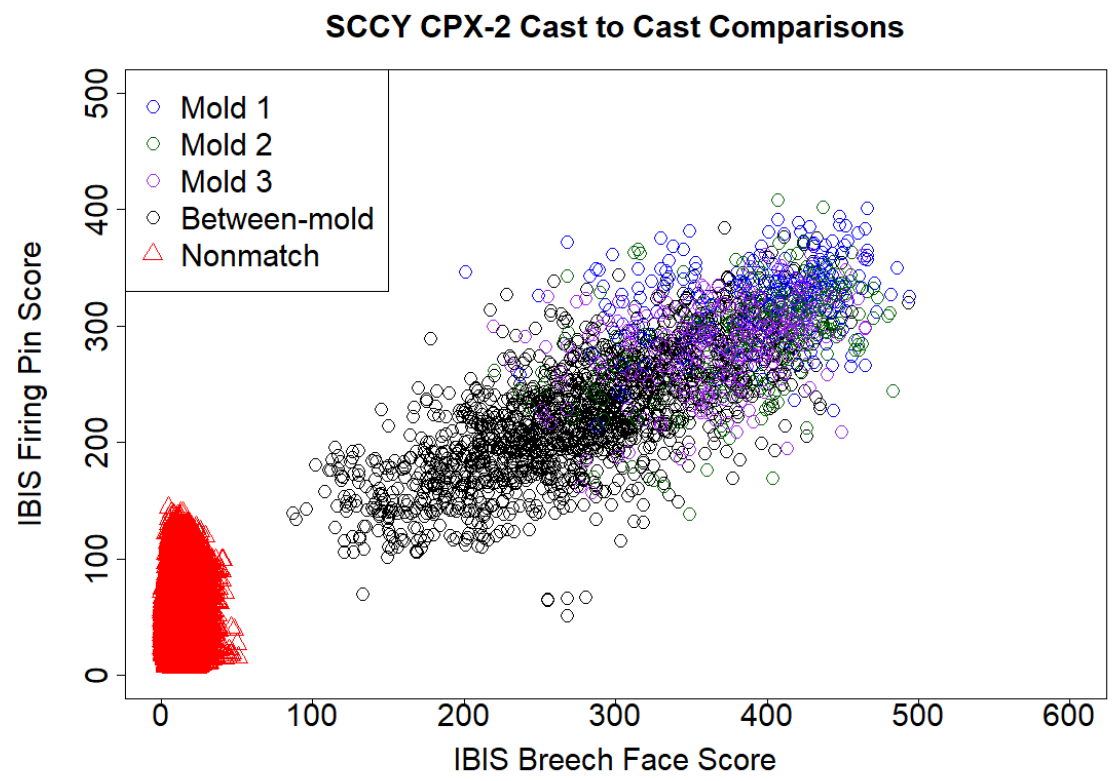

Figure 10: Scatterplot of the SCCY® CPX-2 casts compared to casts.

To illustrate the effect of the inclusion of the mother cartridge cases of the casts, cast-tomaster comparisons are shown in Figures 11 and 12. The cast-to-cast comparisons appear to exhibit the largest scores; however, the cast-to-master scores still demonstrate complete twodimensional separation from the non-matches. The Hi-Point ${ }^{\circledR}$ C9 showed the same trend as the SCCY® CPX-2, but the Smith \& Wesson ${ }^{\circledR}$ SD9VETM was different from the other two firearms and is shown in Figure 12. Visually, the cast-to-master firing pin comparison scores were lower than the cast-to-cast firing pin scores for the Smith \& Wesson ${ }^{\circledR}$ SD9VE ${ }^{\mathrm{TM}}$. The low firing pin scores are also apparent in the boxplots, where the Smith \& Wesson ${ }^{\circledR}$ SD9VE ${ }^{\mathrm{TM}}$ produced the lowest firing pin scores when compared to the other two firearms. It is necessary to note that the Smith \& Wesson ${ }^{\circledR}$ SD9VETM has a metal injection molded firing pin that has a high potential for leaving subclass characteristics [10]. Therefore, firearm examiners may not find the firing pin impression to be as useful as marks present on the breech face. 


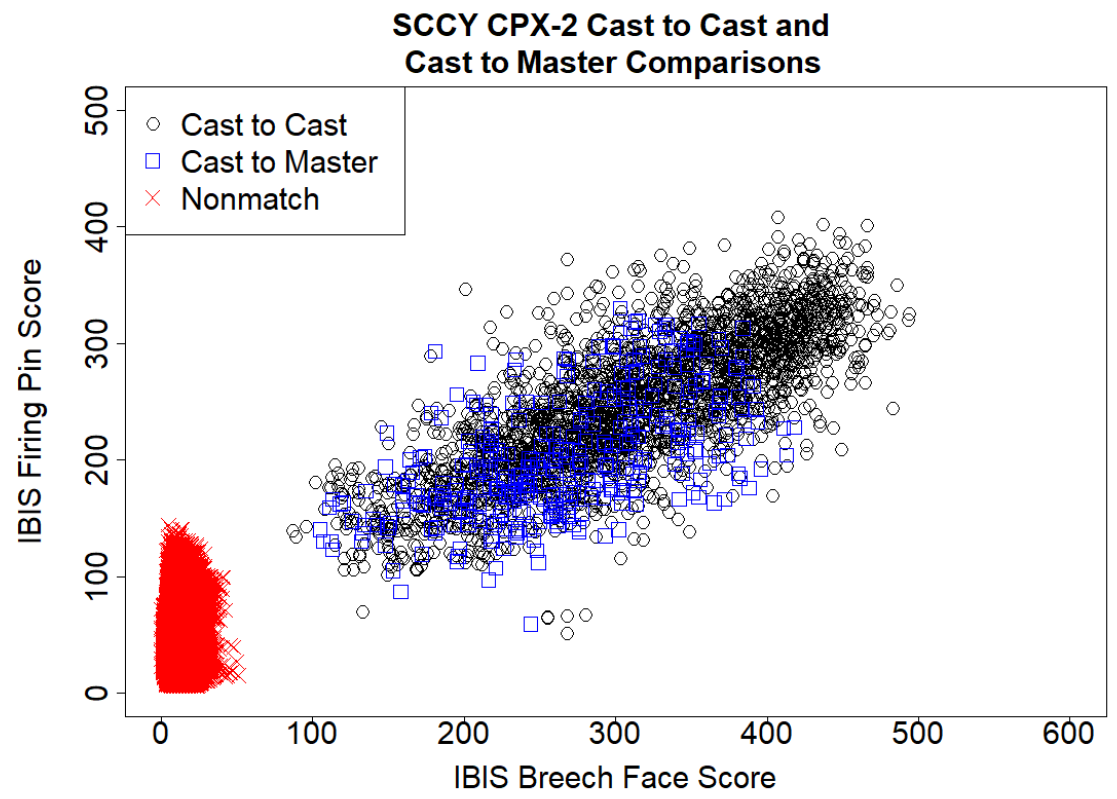

Figure 11: Scatterplot of the $S C C Y^{\circledR} C P X-2$ casts compared to casts and casts compared to their master cartridge cases.

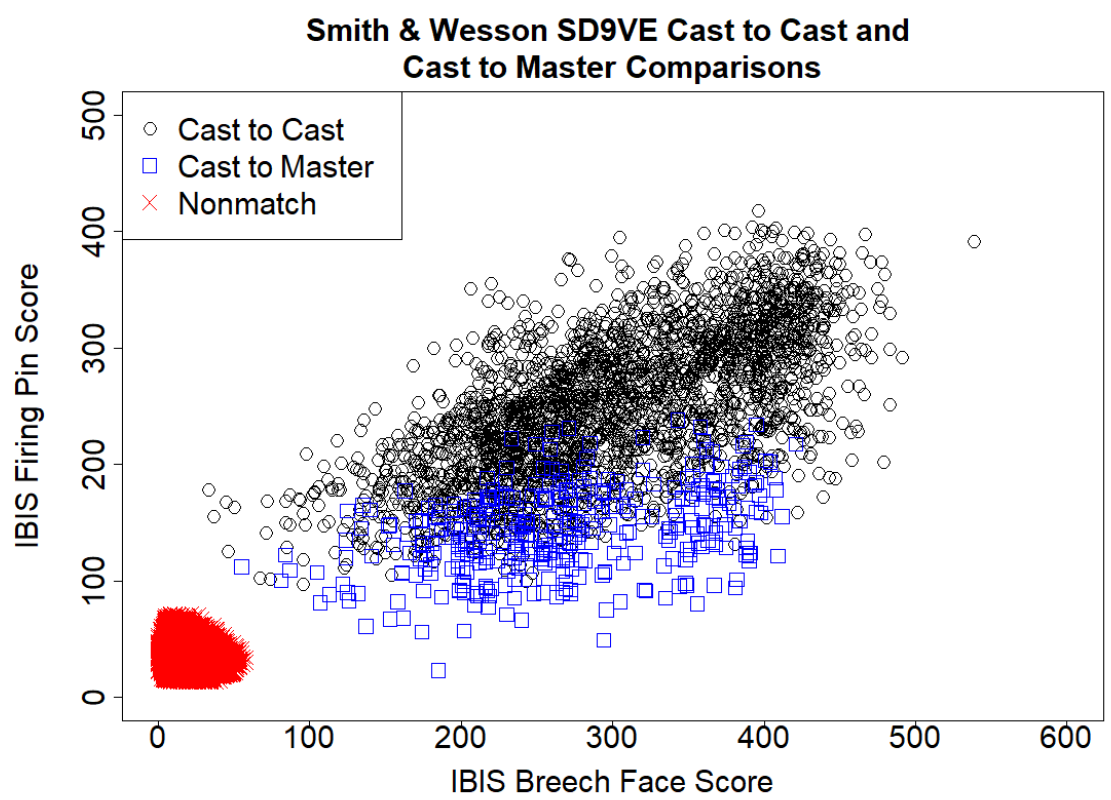

Figure 12: Scatterplot of the Smith \& Wesson ${ }^{\circledR}$ SD9VE ${ }^{\mathrm{TM}}$ casts compared to casts and casts compared to their master cartridge cases.

The plot displayed in Figure 10 is similar to what was observed in Figure 2 of another study using five NIST standard cartridge cases [5]. Similar variation in IBIS ${ }^{\circledR}$ scores was seen when five separate NIST standard cartridge cases were entered into the same IBIS ${ }^{\circledR}$ used for the casts. The 
maximum scores were larger with the NIST standard cartridge cases than with the SCCY ${ }^{\circledR}$ CPX2, Hi-Point ${ }^{\circledR}$ C9, and Smith \& Wesson ${ }^{\circledR}$ SD9VETM casts; however, the master cartridge case selected by NIST for replication to create the NIST standard cartridge cases was chosen, at least in part, on the prevalence of marks. Within the cartridge cases fired by the SCCY ${ }^{\circledR} \mathrm{CPX}-2, \mathrm{Hi}$ Point ${ }^{\circledR}$ C9, and Smith \& Wesson ${ }^{\circledR}$ SD9VETM used to create the casts there is variation in the quantity and quality of marks present which may lead to a decrease in scores when compared to the NIST standard; however, in two dimensions there is complete separation with all casts and with the NIST standards.

The differences observed where the cast-to-master comparisons exhibit lower IBIS ${ }^{\circledR}$ similarity scores than cast-to-cast comparisons is also seen in the NIST cast IBIS ${ }^{\circledR}$ results (Figure 5). The master NIST standard cartridge cases are grouped at lower similarity scores than when the NIST standard casts were compared to each other. This similarity in the plots further shows that the lower scores may result because of the comparison of black plastic to metal. Because of the different materials, the IBIS ${ }^{\circledR}$ may be picking up on lighting differences between the casts and master cartridge cases.

\section{Double Cast Variability Visual Comparisons}

To evaluate the decreasing trend observed in the boxplots, comparison images were extracted from the IBIS ${ }^{\circledR}$ (Figure 13). The first image is one of the master cartridge cases from the SCCY ${ }^{\circledR}$ CPX-2, and the following images are casts of that mother cartridge case. The images of the casts are displayed in order based on mold and set numbers. For example, the first cast image is from Mold 1, Set 1, and the sixth cast image is from Mold 2, Set 1 . The IBIS ${ }^{\circledR}$ breech face score is also displayed on each cast image that represents the comparison of the cast in that image to the master shown in the first image. Observation of the casts through the comparison microscope shows some small differences in the surfaces. However, variation in the corresponding IBIS ${ }^{\circledR}$ scores is quite large. The first cast image (Mold 1, Set 1) has a large defect on the left side of the breech face, however, the breech face score given by IBIS ${ }^{\circledR}$ was 266 . This score is larger than all but one of the scores given to the casts from Mold 2 and Mold 3 that are as low as 126. As mentioned previously, IBIS ${ }^{\circledR}$ scores were used here as a simple similarity metric.

Comparison images were also taken of the same cartridge case and casts as in Figure 13 using a Leica ${ }^{\circledR}$ FS C comparison microscope and a Nikon ${ }^{\circledR}$ D5200 DSLR camera. Both the 
aperture shear (Figure 14) and opposite side of the breech face from the aperture shear (Figure 15) were imaged at $100 \times$ magnification. The images are organized in the same fashion as the IBIS $^{\circledR}$ images in Figure 13 with the master cartridge case being the first image and the following images being the casts in order based on mold and set numbers. There do not appear to be any major differences in the aperture shear images (Figure 14) between any of the casts compared to each other or to the master cartridge case. The individual striations are reproduced well in each of the casts, as are the granular breech face marks.

Interestingly, the first three cast images in Figure $\mathbf{1 5}$ of the breech face area opposite the aperture shear have artifacts that are not present on the master cartridge case. The first cast image (from Mold 1, Set 1) has the defect that is also visible in the IBIS $^{\circledR}$ comparison images (Figure 13) but was given a correlation score of 266 when compared to the master cartridge case. The second and third cast images (from Mold 1 Set 2 and Set 3) also have some foreign materials on them but still received IBIS ${ }^{\circledR}$ correlation scores of 305 and 351, respectively. Again, these are larger scores than given to any of the casts from Mold 2 and Mold 3 that did not have these artifacts present. Based on visual examinations of the images in Figures 14 and 15, overall, the fine markings are reproducible from cast to cast, regardless of the scores given by the IBIS ${ }^{\circledR}$. 

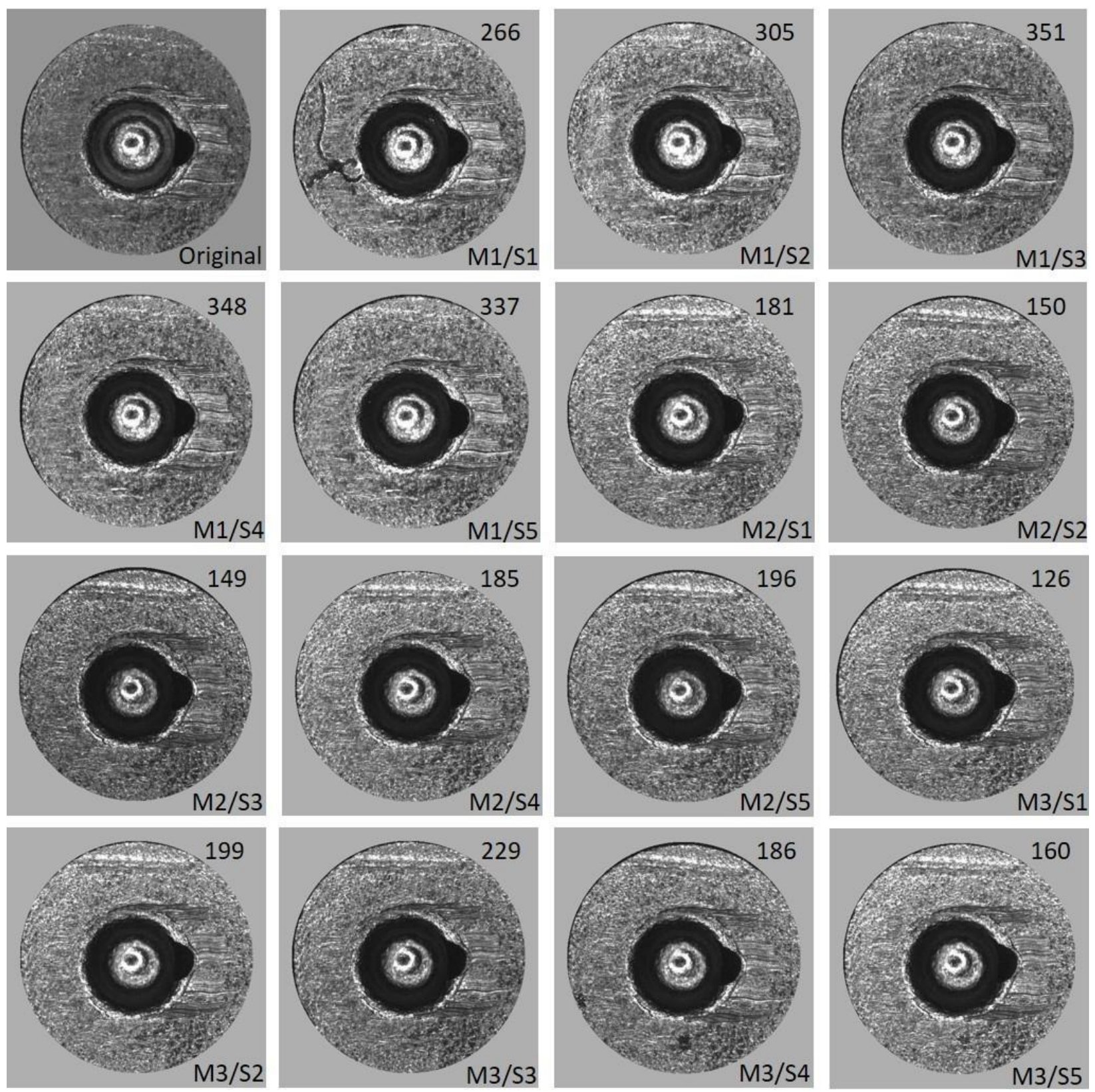

Figure 13: Images from $I B I S^{\circledR}$ of $S C C Y^{\circledR} C P X-2$ cast variability. The number in the top right corner of each image is the IBIS ${ }^{\circledR}$ breech face score of the cast compared to the master cartridge case. Each cast is labeled with the mold (M) and set (S) number. 

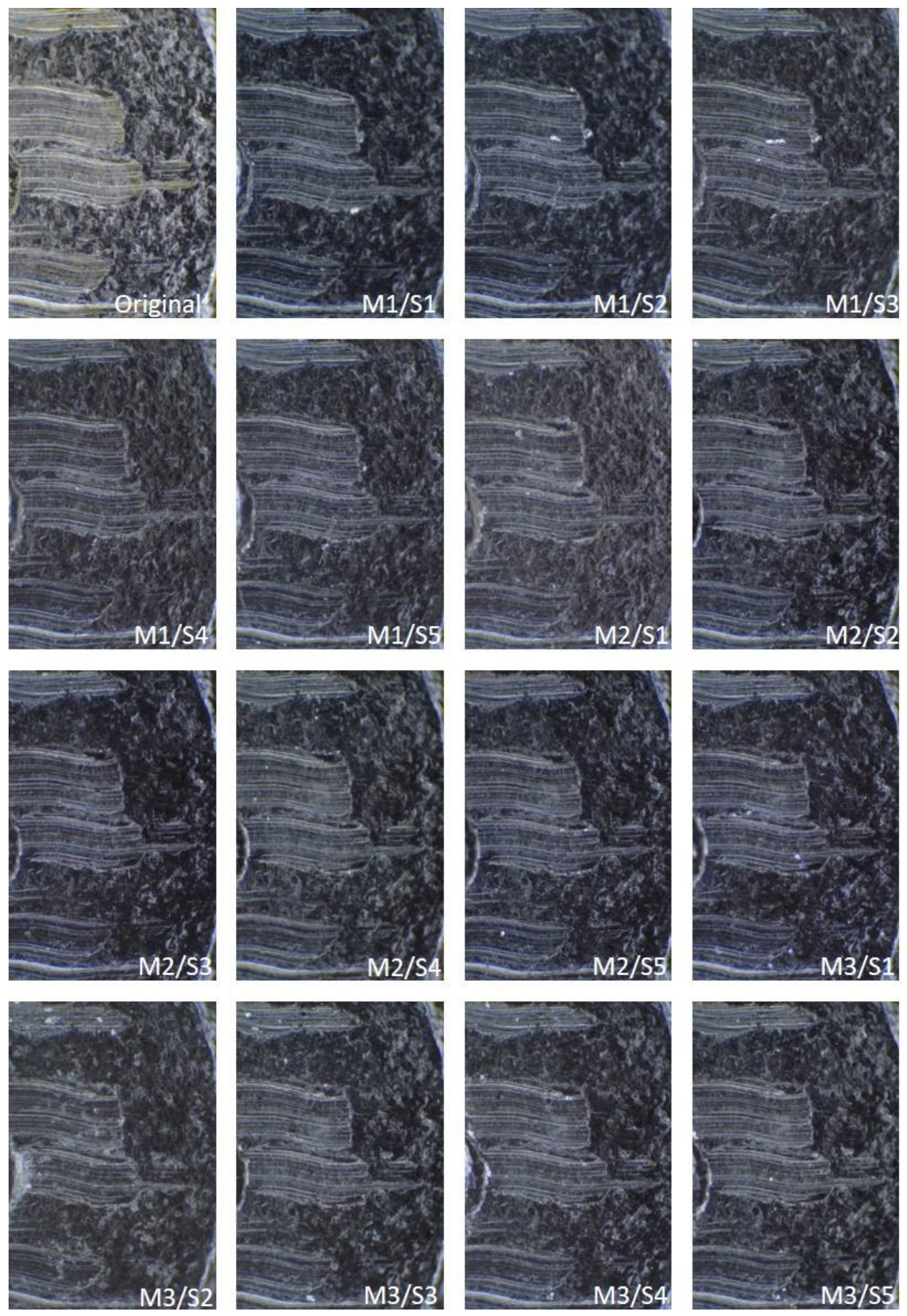

Figure 14: Images of the aperture shear of $S C C Y^{\circledR} C P X-2$ cartridge case casts at 100X magnification using a Leica ${ }^{\circledR}$ FS C comparison microscope. An LED ring light was used for illumination, and images were taken with a Nikon ${ }^{\circledR}$ D5200 camera. Each cast is labeled with the mold $(M)$ and set $(S)$ number. 

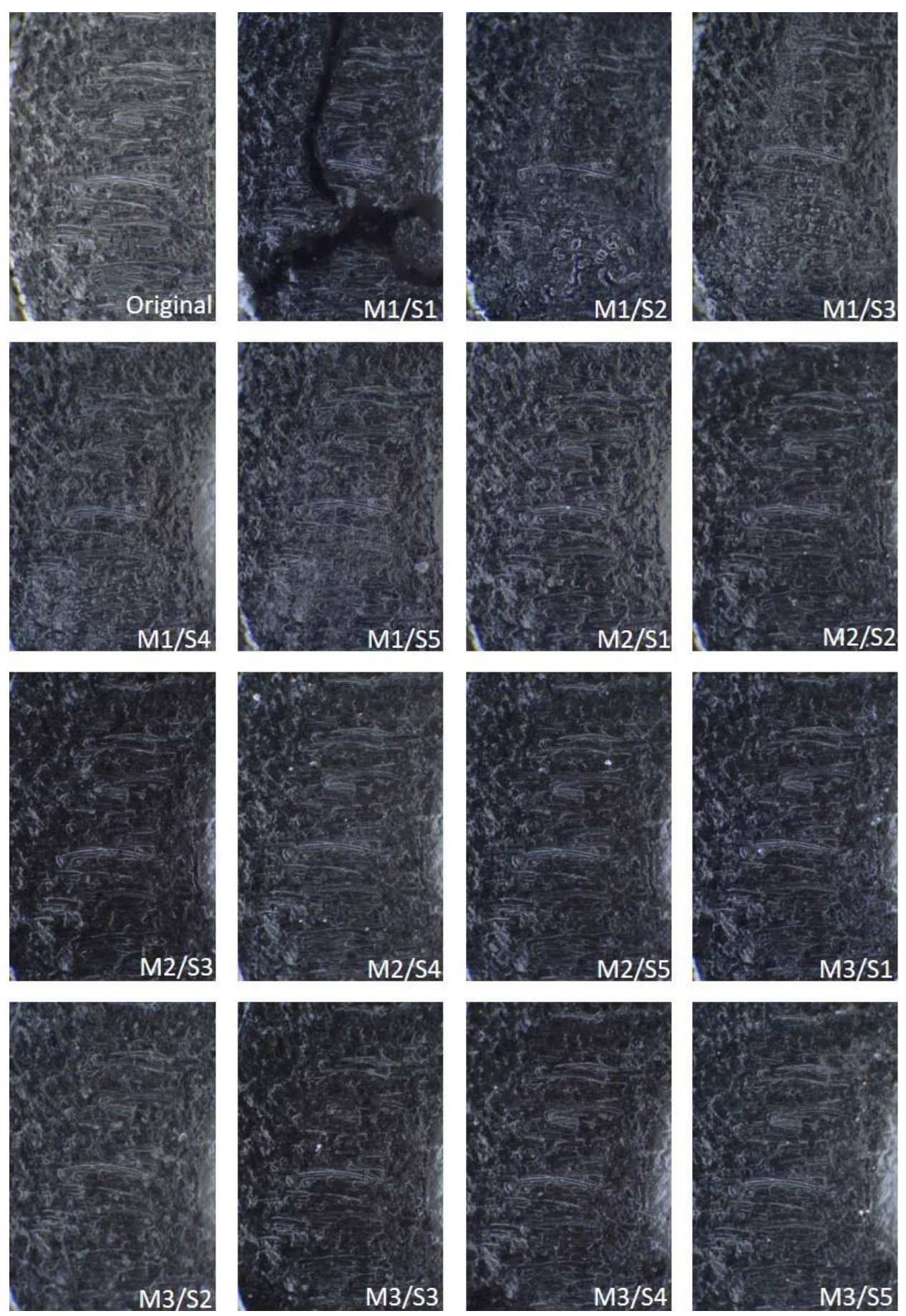

Figure 15: Images of the left side of the breech face of SCCY ${ }^{\circledR} C P X-2$ cartridge case casts at 100X magnification using a Leica ${ }^{\circledR}$ FS C comparison microscope. An LED ring light was used for illumination, and images were taken with a Nikon ${ }^{\circledR}$ D5200 camera. Each cast is labeled with the mold $(M)$ and set $(S)$ number. 
Low IBIS ${ }^{\circledR}$ scores (lower than those seen in casts from the first mold) may be the result of the cross-material comparison because the impressions on black plastic casts are being compared to the impressions on nickel primers. However, even when comparing casts to other casts, some instances of low scores can be seen. In Figure 10, for example, one of the lowest score combinations was a breech face score of 133 and a firing pin score of 70. Figure 16 shows the appearance of each cast that resulted in the low breech face score, and Figure 17 shows the appearance of each cast that resulted in the low firing pin score. From these images, it is clear that the detail from the master cartridge case was reproduced in both of these casts, even though IBIS ${ }^{\circledR}$ assigned one of the lowest cast-to-cast similarity scores.

The comparison images displayed above are all from cartridge cases and casts from the SCCY ${ }^{\circledR}$ CPX-2 pistol; however, similar trends with the images shown were observed with both the Hi-Point ${ }^{\circledR} \mathrm{C} 9$ and Smith \& Wesson ${ }^{\circledR}$ SD9VETM cartridge cases and casts.
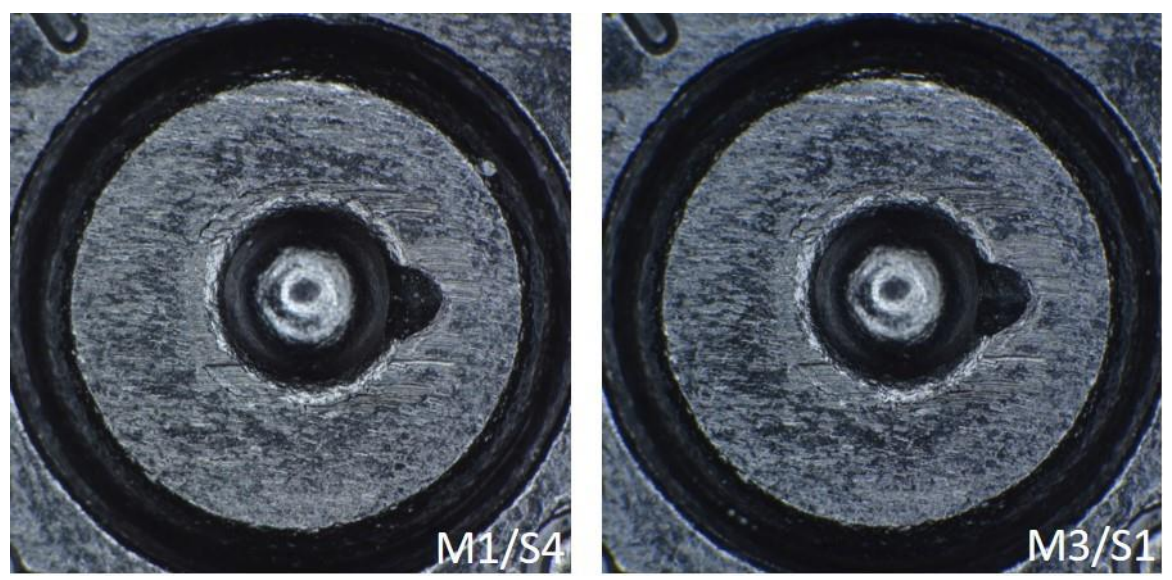

Figure 16: The IBIS ${ }^{\circledR}$ breech face score for this cast to cast comparison from a SCCY ${ }^{\circledR} C P X-2$ was 133. Each cast is labeled with the mold $(M)$ and set $(S)$ number. Images taken at $20 \times$ magnification using a Leica ${ }^{\circledR}$ FS C comparison microscope, LED ring lights, and a Nikon ${ }^{\circledR}$ D5200 camera. 

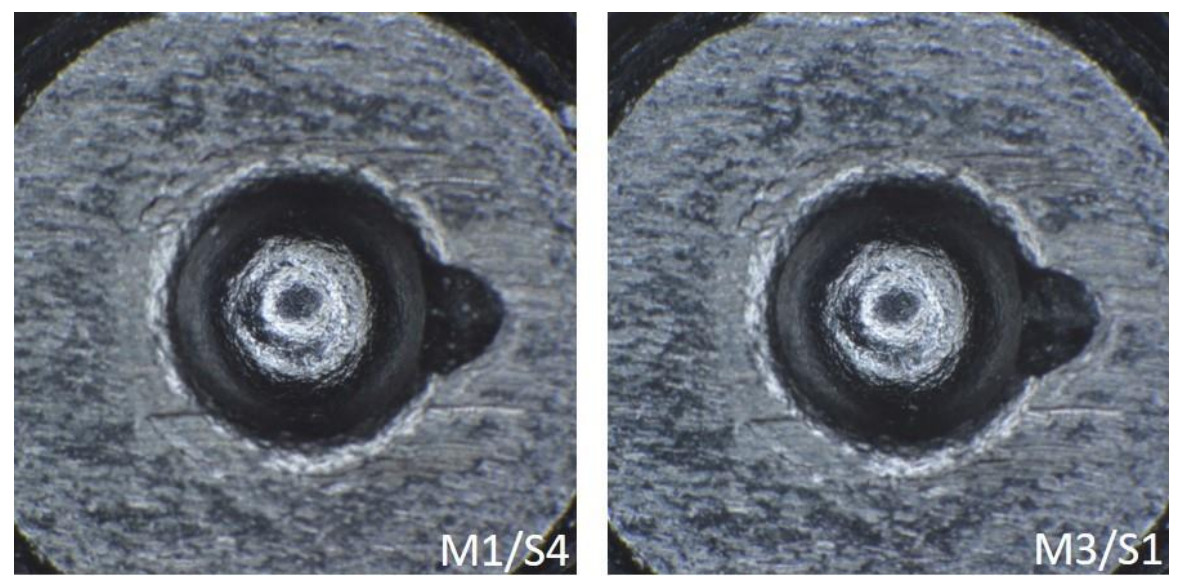

Figure 17: The IBIS ${ }^{\circledR}$ firing pin score for this cast to cast comparison from a $S C C Y^{\circledR} C P X-2$ was 70. Each cast is labeled with the mold $(M)$ and set $(S)$ number. Images taken at $30 \times$ magnification using a Leica ${ }^{\circledR}$ FS C comparison microscope, LED ring lights, and a Nikon ${ }^{\circledR}$ D5200 camera.

\section{$\underline{\text { Limitations }}$}

Two-dimensional grayscale image comparisons were used for the similarity scores. This type of image comparison is, at least in part, based on light intensity being reflected off of the surface. Because the metal primers and black plastic reproductions are different surfaces, light reflects off of them differently. This may be some of the cause of the variability observed in the IBIS $^{\circledR}$ scores.

Although bullets were not used, the same method should be able to be applied. The new standard bullet being made by NIST (SRM 2460a) is a polyurethane cast created from a mold that is subsequently plated with nickel and gold, and it has been shown to be similar in topography to the original standard bullet (SRM 2460) [11].

\section{Conclusions}

Double-casting has been shown to be reliable and accurate in creating cartridge case reproductions. Even though there were statistically significant differences in the IBIS ${ }^{\circledR}$ data between the molds and sets when the casts were compared to their master cartridge cases, the castto-cast comparison data shows complete separation from the non-matches and has a similar trend as observed in a previous study with five NIST standard cartridge cases [5]. Furthermore, in terms of practical significance, visual examinations of the casts supports the ability of double-casting to 
reproduce fine detail and demonstrates the technique's usefulness for comparisons, even when multiple molds and sets are utilized.

Double-casting offers several potential advantages to the field of firearm and toolmark examination and identification. One such advantage is its applicability to training. Laboratories could create their own training sets of cartridge case casts so that all examiners in the class are examining the same exhibits, rather than cartridge cases fired by the same firearm but with varying levels of marking reproduction. Another advantage is the standardization of proficiency test samples. The level of difficulty for particular sets, and the range of difficulty for the entire test, would be able to be standardized by replicating sets of fired cartridge cases to distribute to all examiners. ENFSI (European Network of Forensic Science Institutes) has been using double-cast proficiency test samples for many years [12]. A third advantage is for the study of subclass characteristics. For example, cartridge cases fired by two different Smith \& Wesson ${ }^{\circledR}$ SW 40VE Sigma pistols were shown to have subclass carryover [13]. These cartridge cases, or others found to have subclass carryover, could be replicated using double-casting and sent to others in the firearm examiner community for comparisons. This would allow examiners to have many examples of best known non-matches from other examiners in the community.

\section{Acknowledgements}

The authors would like to acknowledge Dr. Casey Jelsema for his support with the statistical analysis. The authors would also like to acknowledge Mr. Barrett Towns for his assistance in making the molds and casts, as well as entering exhibits into the IBIS® database.

\section{References}

[1] D. F. Seiler, R. L. Watters Jr., NIST Certificate: Standard Reference Material 2461 Standard Cartridge Case, NIST, 2012.

[2] J. Song, E. Whitenton, D. Kelley, R. Clary, L. Ma, S. Ballou, M. Ols, SRM 2460/2461 Standard Bullets and Casings Project, Jour-1 of Research of the National Institute of Standards and Technology 109 (2004) 533-542.

[3] J. Song, P. Rubert, A. Zheng, T. Vorburger, Topography measurements for determining the decay factors in surface replication, Measurement Science and Technology (2008). 
[4] INTERPOL, INTERPOL Ballistics Information Network: Handbook on the Collection and Sharing of Ballistics Data, 3rd edition, 2014.

[5] K. B. Morris, E. F. Law, R. L. Jefferys, E. C. Dearth, E. B. Fabyanic, An evaluation of the discriminating power of an Integrated Ballistics Identification System Heritage System with the NIST standard cartridge case (standard reference material 2461), Forensic Science International 280 (2017) 188-193.

[6] W. J. Conover, Practical Nonparametric Statistics, Wiley Series in Probability and Mathematical Statistics, John Wiley and Sons, 2nd edition, 1980.

[7] W. J. Conover, R. L. Iman, On multiple-comparisons procedures, Tech. Rep. LA-7677-MS, Los Alamos Scientific Laboratory, 1979.

[8] R Core Team, R: A Language and Environment for Statistical Computing, R Foundation for Statistical Computing, Vienna, Austria, Version 3.5.0, 2018, ISBN: 3-900051- 07-0.

[9] T. Pohlert, PMCMRplus: Calculate pairwise multiple comparisons of mean rank sums, R package version 1.0.1, 2018.

[10] R. Nichols, Subclass characteristics: from origin to evaluation, AFTE Journal 50 (2018).

[11] D. Gundlach, S. J. Choquette, NIST Certificate: Standard Reference Material 2460a Standard Bullet Replica, NIST, 2018.

[12] P. Pauw-Vugts, A. Walters, L. Øren, Kripos, and L. Pfoser, FAID2009: Proficiency Test and Workshop, AFTE Journal, Vol. 45, No. 2, Spring 2013, pp. 115-127.

[13] G. Rivera, Subclass characteristics in Smith \& Wesson SW40VE Sigma pistols, AFTE Jour139 (2007). 


\title{
3. Three-dimensional analysis of cartridge case double-casts
}

\section{Publication status}

This chapter has been published in the Journal of Forensic Sciences. Citation: Eric F. Law and Keith B. Morris. Three-dimensional analysis of cartridge case double-casts. Journal of Forensic Sciences, September 2020 (awaiting journal volume and page numbers).

\begin{abstract}
Due to the shot-to-shot variability in tool mark reproduction on fired cartridge cases, a method of replication is needed for the creation of training and testing sets. Double-casting is one method that has been used for this application, but the accuracy and variability of this method needs to be characterized. Three firearms were used to fire 25 cartridges each to create the master cartridge cases. The double-casting method consists of creating a silicone mold of the master cartridge case. A plastic resin mix is then poured into the mold to create the double-cast reproduction. Fifteen double-casts of each of the 75 fired cartridge cases were created across different silicone molds to analyze within- and between-mold variability. The master cartridge cases and doublecasts were scanned with a confocal microscope (Sensofar ${ }^{\circledR} \mathrm{S}$ neox) to create three dimensional representations of the surfaces. Two similarity metrics were used for the objective comparison of the double-casts to their master cartridge cases: the areal correlation coefficient ( $\left.\mathrm{ACCF}_{\mathrm{MAX}}\right)$ and the number of congruent matching cells (CMC). The $\mathrm{ACCF}_{\mathrm{MAX}}$ and $\mathrm{CMC}$ data, along with visual examinations, showed that the double-casting method produces accurate reproductions. Withinmold variability was found to be minimal, and between-mold variability was low. These results illustrate that double-casting can be applied for training and testing purposes.
\end{abstract}




\section{Introduction}

Breech face and firing pin impressions on fired cartridge cases appear differently from shot to shot due to variability in reproduction through the firing process. Due to this variability, a method of replication is necessary to create training and testing sets. The National Institute of Standards and Technology (NIST) maintains a standard cartridge case, standard reference material (SRM) 2461, that is a reproduction of a master cartridge case created through electroforming (1). NIST has used confocal microscopy to acquire 3D scans of their reproduced standard cartridge case to compare to the master cartridge case (2).

Plastic replicas have been used, and the methods published on, as far back as 1956 with high detail resolution (3). More recently, this method has been called double-casting, where silicone mold negatives are made from master cartridge cases, and plastic replicas are created using the molds (4). The European Network of Forensic Science Institutes (ENFSI) has been using double-cast samples in proficiency tests for the purpose of having all participants examine the same samples (5). Visual comparisons between casts and their master cartridge cases have verified that the method produces high quality reproductions (5). Recently, NIST created the SRM 2460a Standard Bullet that is a polyurethane cast coated in a fine layer of metal to increase surface reflectivity and better represent an actual fired bullet (6).

However, there is expected variability in the reproduction of the fine detail from the master cartridge case when creating multiple double-casts from a single mold, or multiple double-casts of the same cartridge case from different molds. This variability needs to be characterized to ensure each double-cast is representative of the master cartridge case. An IBIS® Heritage ${ }^{\mathrm{TM}}$ system has been used for this purpose in a previous study (7). Those results were based on 2D grayscale images and therefore lighting differences between the master cartridge cases (metal) and doublecasts (black plastic), due to differences in surface reflectivity, may have affected similarity scores.

To further evaluate the accuracy of double-cast tool mark reproduction compared to their master cartridge cases, confocal microscopy was used to measure the surfaces. This allowed for the depths of the impressions and striations to be directly considered. Confocal microscopy is also less dependent on lighting than conventional 2D imaging, and therefore effects of surface reflectivity differences will be reduced. The goal of this study was to analyze the variability in the level of detail reproduced through the double-casting process using the areal correlation coefficient $\left(\mathrm{ACCF}_{\mathrm{MAX}}\right)$ and the congruent matching cells $(\mathrm{CMC})$ algorithm developed by NIST. The breech 
face impression area will be the focus of this study because CMC is currently optimized for the breech face area. The overall similarity between surfaces using the ACCFMAX and the number of congruent cells were used to provide an objective measure of similarity between double-casts and their master cartridge cases.

\section{Material and Methods}

Twenty-five cartridge cases were fired from each of the three firearms to produce master cartridge cases for double-casting: SCCY® CPX-2, Hi-Point ${ }^{\circledR}$ C9, and Smith \& Wesson ${ }^{\circledR}$ SD9VE. These firearms were selected based on the types of tool marks they produce on the breech faces of fired cartridge cases. The CPX-2 produces prominent aperture shear, the $\mathrm{C} 9$ leaves parallel impressions, and the SD9VE imparts distinct granular impressions. One cartridge case from each of these firearms is shown in Fig. 1.
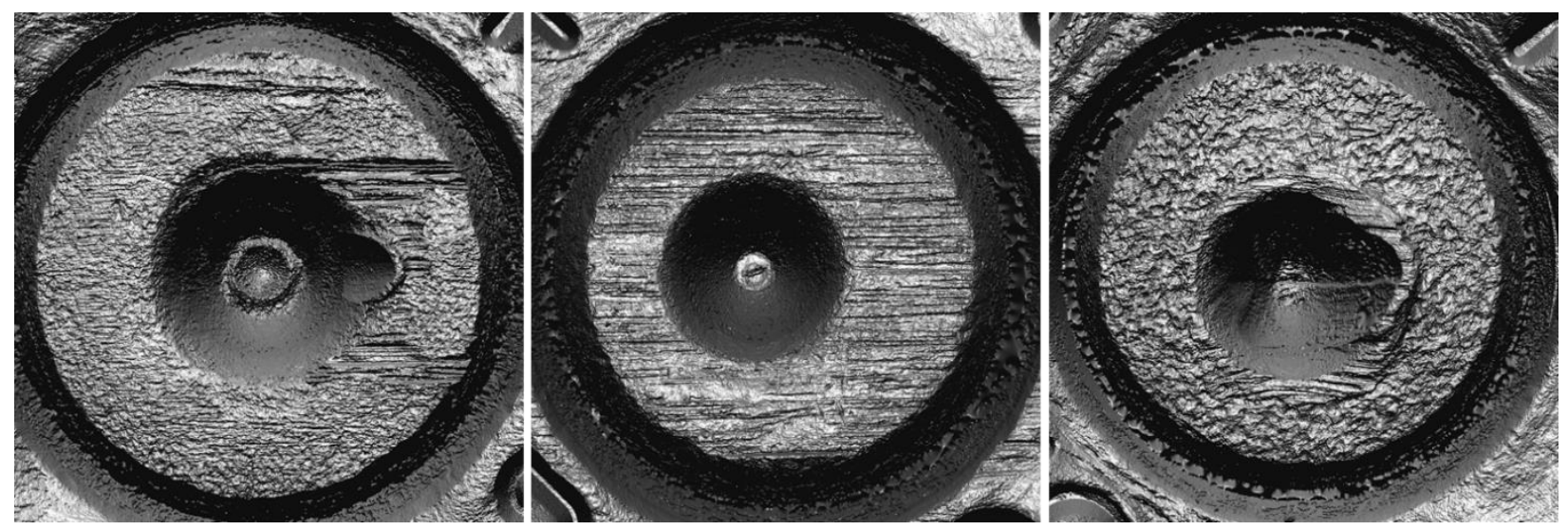

FIG. 1-Examples of cartridge cases from each of the three firearms. Left is from the SCCY® $C P X-2$, middle is from the Hi-Point ${ }^{\circledR} C 9$, and right is from the Smith \& Wesson $₫$ SD9VE. Images saved from the Cadre X3P viewer (8) with the enhanced contrast option enabled.

Fifteen double-casts were created from each of the 25 cartridge cases fired by each of the three firearms. This resulted in 1,125 double-casts. To make the silicone molds for each firearm, the 25 master cartridge cases were arranged on a custom 3D printed holder (Fig. 2). The cartridge cases were then covered with a two-part liquid silicone mix, Smooth-On® Mold Star ${ }^{\mathrm{TM}}$ 30. The mix was placed into a pressure pot for curing at 45 psi to remove air bubbles from the mixture at room temperature for approximately six hours. The result was a single cylindrical piece of silicone 
that was approximately $11.5 \mathrm{~cm}$ in diameter and $4.0 \mathrm{~cm}$ in thickness (Fig. 2). This was repeated three times for the master cartridge cases from each firearm for a total of three molds. After the mold had cured and the master cartridge cases were removed, a two-part plastic resin mix, SmoothOn® Smooth Cast ${ }^{\circledR}$ 327, was dyed black with Smooth-On® SO-Strong ${ }^{\circledR}$ black colorant and poured into the mold. This plastic resin mix was also cured in the pressure pot at 35 psi at room temperature for approximately six hours. After the plastic resin finished curing, each double-cast was removed from the mold. This process was repeated to create five double-casts of each master cartridge case from each of the three molds per firearm, for a total of 15 double-casts per master cartridge case. Fig. 2 displays five reproductions of Master Cartridge Case \#1 created from Mold 1 for one firearm. This allowed for the analysis of within and between mold variability. This method has also been described in a previous study where an IBIS® Heritage ${ }^{\mathrm{TM}}$ system was used to evaluate the reproducibility of the same firearms, molds, and double-casts as those used in this study (7).

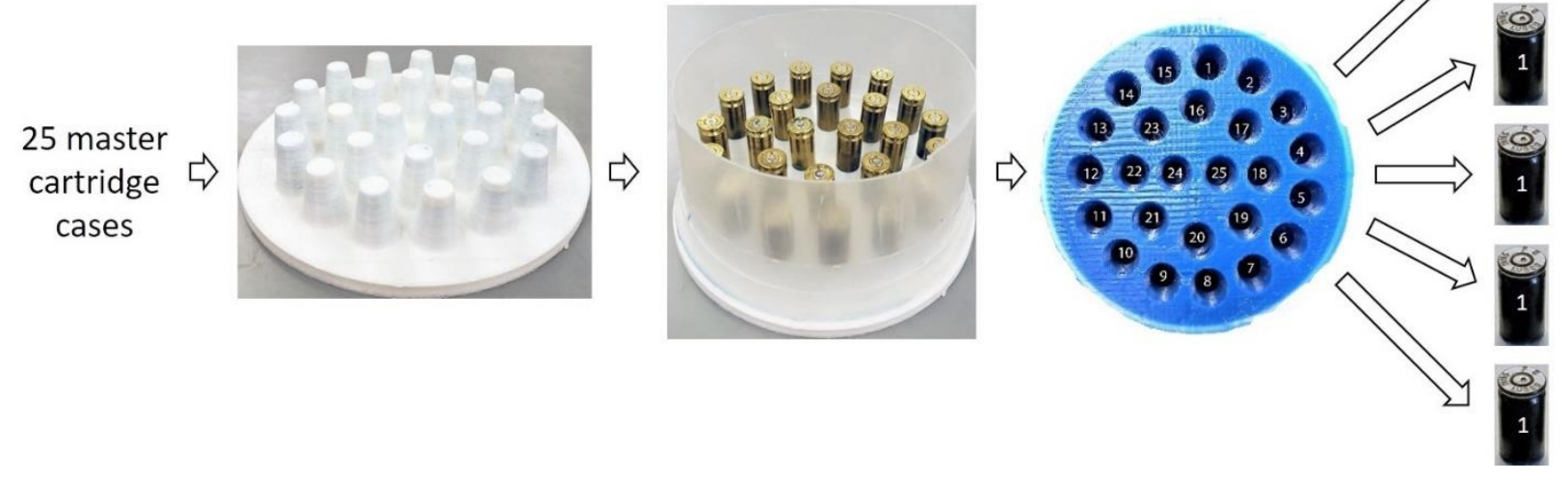

FIG. 2-Illustration of the double-casting method.

A Sensofar ${ }^{\circledR}$ S neox optical profiler was used to acquire three-dimensional scans of the fired master cartridge cases as well as all the reproductions using the "Confocal Fusion" scan mode. This is a proprietary method developed by Sensofar ${ }^{\circledR}$ that primarily measures a surface using confocal microscopy, however, if a confocal data point is not measured at a given location then focus variation is used instead (e.g. areas with steep slopes). Generally, this method works well. It is difficult to measure the edges of the firing pin impression even with this method, although this area is not typically of interest in cartridge case comparisons. The scans were all 
converted to the *.x3p file format for software interoperability and ease of use (9). All files were named in a way that allowed for the specific mold number, cast set number, and master cartridge case number to be known. An example filename is "DAN-FA-UNK-0203-0001", where each part is defined as:

- DAN: unique letter combination that identifies these as double-casts of the CPX-2 master cartridge cases.

- FA: Federal American Eagle manufactured ammunition was used.

- UNK: not applicable here because manufactured ammunition was used but can be used to refer to the primer and powder of a reloaded cartridge.

- 0203: identifies this double-cast was created from the second mold and was made in the third set of double-casts created from that mold.

- 0001: refers to the first cartridge case of the 25 masters fired from this firearm.

Two similarity metrics were used for the objective comparison of the double-casts to their master cartridge cases: the areal correlation coefficient ( $\mathrm{ACCF}_{\mathrm{MAX}}$ ) and the number of congruent matching cells (CMC). The comparison software was developed by NIST and was provided to the authors for research purposes as part of an ongoing collaboration. The CMC method has been described in detail elsewhere (10-12) but will be explained in brief here. To prepare the *.x3p files for $\mathrm{CMC}$ analysis, the scans were cropped so that only the breech face area remains. Care was taken to select the same breech face areas on the casts as selected on their respective master cartridge cases to reduce comparison effects due to differences in sample domain. To attenuate noise, form, and waviness, a Gaussian Regression filter was applied to each measured surface with $\lambda_{S}=25 \mu \mathrm{m}$ and $\lambda_{C}=400 \mu \mathrm{m}$ cutoff wavelengths. Furthermore, the images were downsampled from their original resolution of $1.38 \mu \mathrm{m} /$ pixel by a factor of two to $2.76 \mu \mathrm{m} /$ pixel. This resulted in faster comparisons while not sacrificing accuracy. One cartridge case scan was then set as the reference surface and another was set as the comparison surface. A grid of $64(500 \times 500 \mu \mathrm{m})$ cells was defined on the reference surface. For each of the cells, a search was performed over all positions of the comparison surface to find the cell registration location that yielded the highest value for the respective cell pair similarity value $\left(\mathrm{ACCF}_{\mathrm{MAX}}\right)$. Each cell was also allowed $360^{\circ}$ rotation during this search. The comparison metric of the CMC method is the number of congruent matching cell pairs, i.e., the number of cell pairs that have both a sufficient similarity and a 
congruent registration location. In this study, to be considered a congruent matching cell, the cell similarity value had to be at least $20 \%$ and the errors in registration position and orientation could not exceed $125 \mathrm{um}$ and $3^{\circ}$, respectively. These criteria are based on research done by NIST (1012), as well as in-house testing of the algorithms. The output included the number of congruent matching cells, as well as an $\mathrm{ACCF}_{\mathrm{MAX}}$ value corresponding to the overall similarity of the two surfaces.

Fig. 3 illustrates the comparison of two cartridge cases fired by the same CPX-2 pistol. The grid of cells, color-coded to show congruency, was overlaid onto the reference surface (Fig. 3, left). The black cells represent a CMC with the comparison surface (Fig. 3, right), while the red cells represent non-CMCs. In the corresponding comparison surface, the cells are displayed based on their best fit locations, as well as color-coded the same as in the reference surface. In this example, the non-congruent cells fall in the aperture shear area of the breech face. Aperture shear is typically present in cartridge cases fired by this particular CPX-2, however, it is not reproducible. The results of this comparison illustrate the effectiveness of utilizing the congruent cell approach. In this comparison an $\mathrm{ACCF}_{\mathrm{MAX}}$ of $33.0 \%$ and 27 congruent cells resulted. This $\mathrm{ACCF}_{\mathrm{MAX}}$ may not be any larger than non-matching comparisons, but 27 congruent cells would lead an examiner to believe these cartridge cases were fired by the same firearm. The highest known non-matching CMC score has never exceeded five in NIST's research (10-12).

Fig. 4 illustrates a CMC comparison with a double-cast of the reference surface used in Fig. 3 (left). As with all double-cast comparisons performed in this study, the master cartridge case was set as the reference surface (Fig. 4, left) and the double-cast was set as the comparison surface (Fig. 4, right). In this instance, all 39 cells from the reference surface were found to be congruent in the comparison surface. 

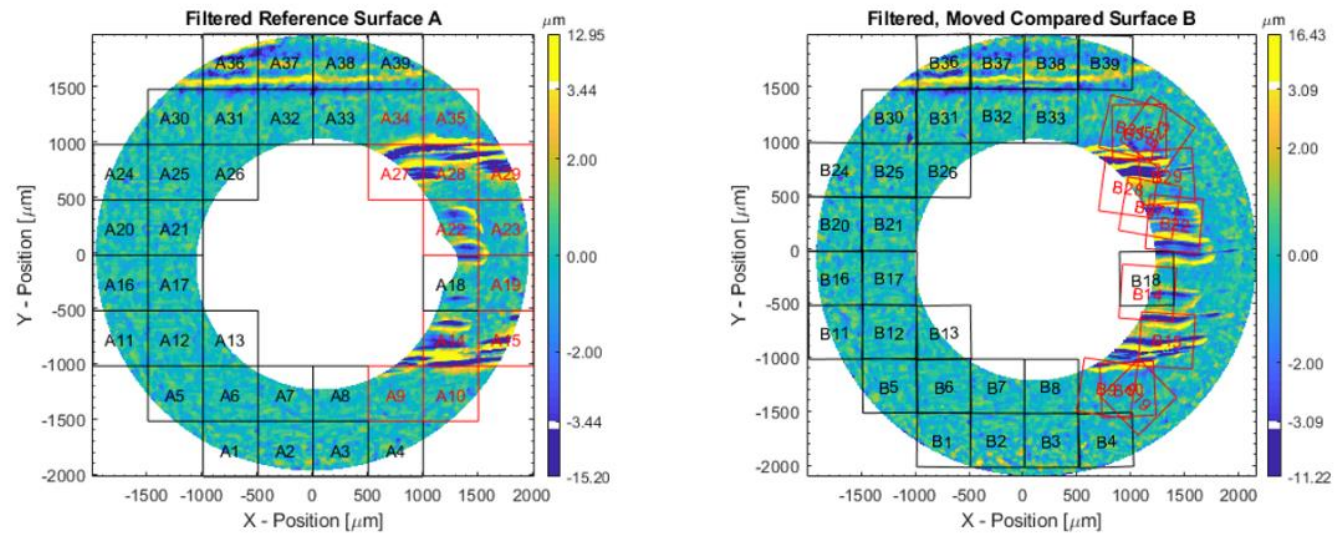

FIG. 3-CMC example for a CPX-2 known match comparison. The overall ACCF ${ }_{M A X}$ was 33.0\%, and there were 27 of 39 congruent cells.
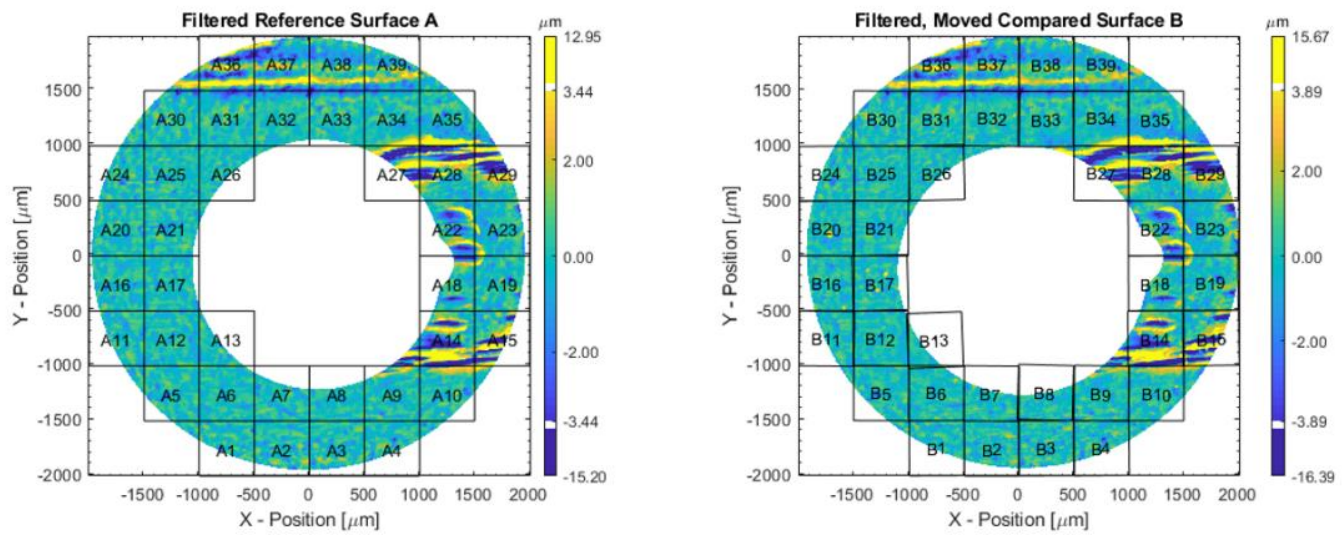

FIG. 4-CMC example for a CPX-2 master cartridge case compared to one of its double-casts. The overall ACCF $F_{M A X}$ was $93.0 \%$ and there were 39 of 39 congruent cells.

The tolerances for congruent cells may appear to be low considering the goal here is to analyze the accuracy of reproductions. It may be expected that individual cells would have greater than $20 \%$ similarity, and less than the allowable $125 \mu \mathrm{m}$ in $\mathrm{x}$ and $\mathrm{y}$ spatial positioning and $3^{\circ}$ in rotation if the double-casts are accurately reproducing the fine detail from the master. However, these criteria were selected to be consistent with comparisons that have previously been run. Using these same criteria allowed for the similarity of the casts to their master cartridge cases versus the comparison of multiple cartridge cases fired by the same firearm to be analyzed, such as in Figs. 3 and 4. Had more strict tolerances been selected for the double-casts, the difference in comparing the reproductions and multiple cartridge cases fired by the same firearm may not have been clear. 
Once all the comparisons of the reproductions to their master cartridge cases had been run, the $\mathrm{CMC}$ and $\mathrm{ACCF}_{\mathrm{MAX}}$ data were organized into Microsoft Excel® spreadsheets. The naming convention was columns corresponding to Cast Set 1.1, Cast Set 1.2, Cast Set 1.3, up to Cast Set 3.5 , where the first number was the mold number $(1,2$, or 3$)$ and the second number was the cast set number from that mold $(1,2,3,4$, or 5$)$. There were 25 values (rows in the data files) within each cast set that represented the similarity of the double-cast compared to its master cartridge case. The Friedman test was then used to compare the cast set groups (the columns in the data files) for each of the firearms to determine if any of the CMC or ACCF significantly different for each of the three firearms. All plots and statistical analyses were performed using $\mathrm{R}$ and RStudio $(13,14)$.

\section{Results and Discussion}

\section{Overall Results}

Comparisons were set up with the master cartridge cases as the reference surfaces and the double-cast scans as the comparison surfaces. The algorithm outputs the overall similarity of the surfaces represented by the $\mathrm{ACCF}_{\mathrm{MAX}}$ as well as the number of congruent cells. Using the raw number of congruent cells with double-casts to investigate the accuracy of the reproductions was slightly misleading. For example, master cartridge case \#1 may have had a larger breech face area than master cartridge case \#2, potentially due to a smaller firing pin impression or a different degree of primer surface contact with the breech face of the firearm. The grid of cells overlaid on master cartridge case \#1 will have more cells than the grid on master cartridge case \#2. During comparison, there are more potential cells to be found congruent in the double-casts of master cartridge case \#1 than with master cartridge case \#2. For this reason, the CMC results displayed are represented as percent values where the number of congruent cells were divided by the total number of cells possible. Descriptive statistics for the number of potential cells for each of the three firearms are shown in Table 1. On average, the CPX-2 had the most potential cells, followed by the SD9VE and C9. 
Table 1: Descriptive statistics for the number of potential cells.

\begin{tabular}{|l|l|l|}
\hline Firearm & Mean & Standard deviation \\
\hline SCCY CPX-2 & 40 & 2 \\
\hline Hi-Point C9 & 33 & 1 \\
\hline Smith \& Wesson SD9VE & 39 & 2 \\
\hline
\end{tabular}

Scatterplots of the results for each firearm are displayed in Fig. 5. The x-axis shows the percent of congruent cells and the y-axis shows the overall similarity ( $\mathrm{ACCF}_{\mathrm{MAX}}$ ). The red squares are the comparison results of the inter-comparisons of the master cartridge cases for the respective firearms. Each blue circle represents a single comparison of a double-cast to its master cartridge case. Each comparison is made up of $n$ cells, each of which have their own similarity, position, and rotation values. The master cartridge case inter-comparisons exhibited larger variability in $\mathrm{CMC}$ and $\mathrm{ACCF}_{\mathrm{MAx}}$ percentages, with the $\mathrm{C} 9$ clearly performing the best of the three firearms. Furthermore, the double-casts all displayed higher similarity than the master cartridge case intercomparisons which was expected. The double-casts generally had $\mathrm{ACCF}_{\mathrm{MAX}}$ values larger than $80 \%$ and a CMC percent above $95 \%$ with all three firearms. Lateral scale differences influence the similarity metrics, more so the $\mathrm{ACCF}_{\mathrm{MAX}}$ due to the position tolerance with the CMC metric. Lateral scale differences may be introduced because of shrinkage of the casts during curing, however, linear shrinkage with the casting material used is $0.0075 \mathrm{in} / \mathrm{in}$, limiting this effect (15). Even so, the values are indicative of the high level of detail reproduced through the double-casting process. 


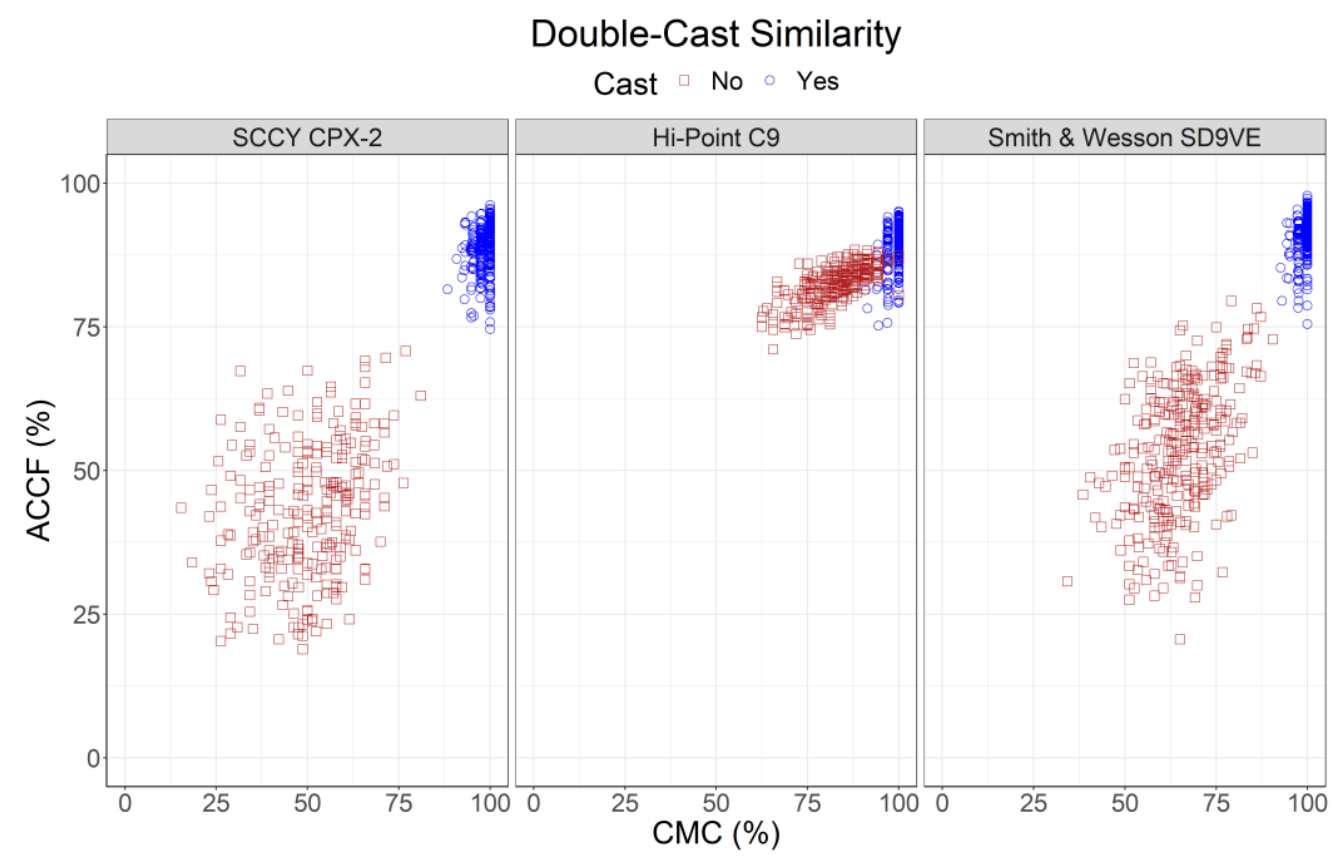

FIG. 5-CMC and ACCF $F_{M A X}$ results for the master cartridge cases compared to their reproductions.

To further investigate the reproduction accuracy, the differences in the number of potential cells on the reference surface (the master cartridge case) and the number of congruent cells in the comparison surface (the double-cast) were plotted and are shown in Fig. 6. It would be expected that all data points should be in the "0" category because the double-casts should be identical to the master cartridge cases. However, due to variation in the casting process there are areas in some of the casts that did not reproduce as well as others and therefore fall outside of the congruency criteria ( $20 \%$ similarity, $125 \mu \mathrm{m}$ in position, $3^{\circ}$ rotation). The CPX-2 had the most cases where the number of CMCs was less than the number of potential cells. The SD9VE had the least, and the C9 results were in between the other two firearms. 


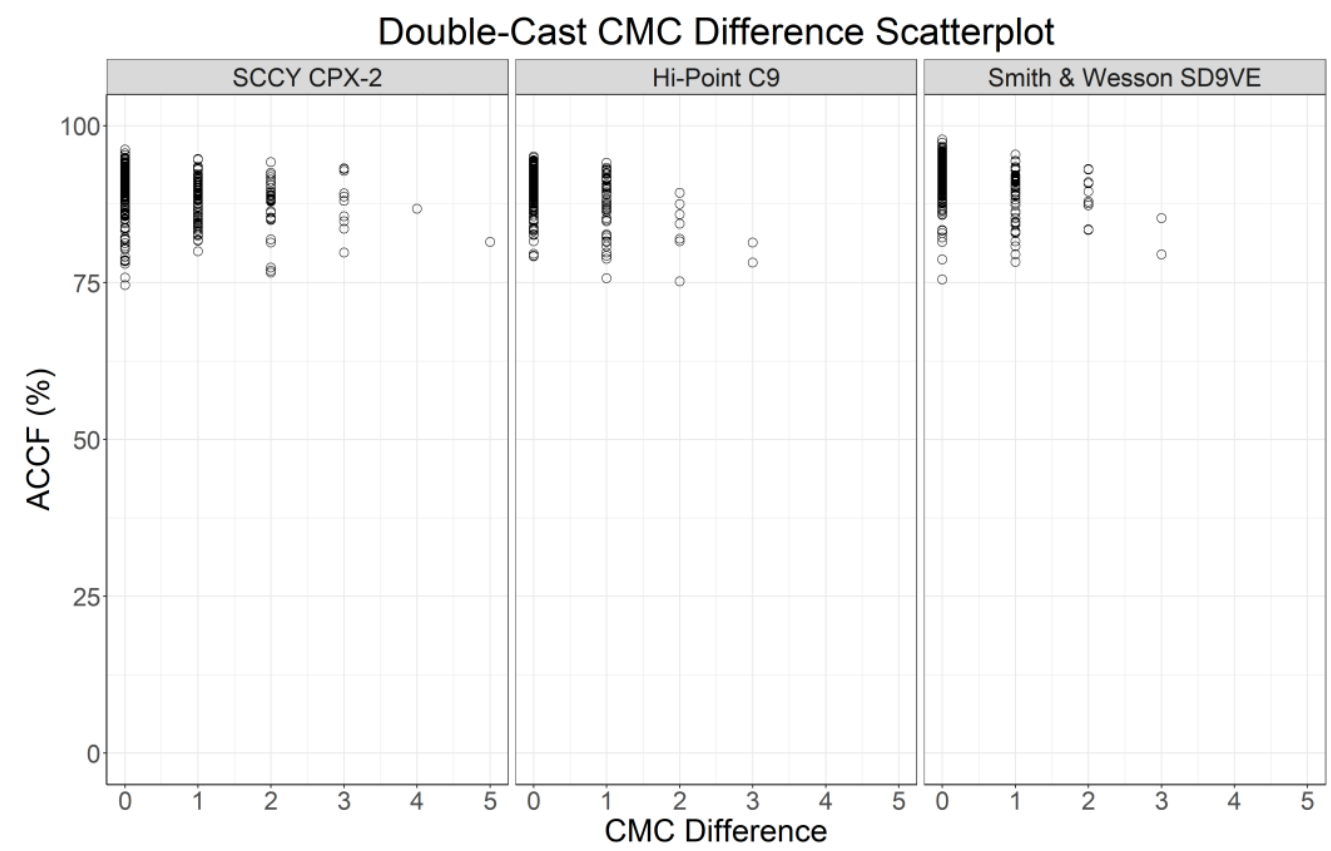

FIG. 6-Scatterplot displaying the number of congruent cells less than the number of potential cells. Any difference in the number of congruent cells means that there were cells that fell outside of the similarity, position, and rotation thresholds.

The Friedman test was used to compare across the mold and set numbers (the columns in the Excel® data files) to determine if there was evidence of significant differences at the 0.05 level of significance (16). The Friedman test is a global test meaning it does not provide information on which groups are different, only if any of the groups are different from any of the others. The $p$ values from this test are shown in Table 2 for both the CMC and ACCF differences, where the $p$-value was less than 0.05, were found in all cases except for the C9 CMC results. For all three firearms there were smaller $p$-values for the ACCFMAX data, indicating more evidence for group differences than with the congruent cell data. Further research is needed to determine the source of this difference. 
Table 2: Friedman test p-values for the three firearms based on both CMC and ACCF $F_{M A X}$ data. Significant differences are highlighted in gray.

\begin{tabular}{|l|l|l|}
\hline Firearm & CMC & ACCF $_{\text {MAX }}$ \\
\hline SCCY CPX-2 & 0.0239 & $<0.0001$ \\
\hline Hi-Point C9 & 0.7613 & $<0.0001$ \\
\hline Smith \& Wesson SD9VE & 0.035 & $<0.0001$ \\
\hline
\end{tabular}

\section{$A C C F_{\text {MAX }}$ Data}

Fig. 7 shows the distributions of $\mathrm{ACCF}_{\mathrm{MAX}}$ values for the different mold and set numbers (x-axis) for each of the three firearms. For example, Cast Set 2.3 refers to Mold 2 and the third set of casts made from that mold. Within each firearm the boxplots all overlap with each other, so based on visual examination, it does not appear that any of the molds produce better or worse reproductions than any others.

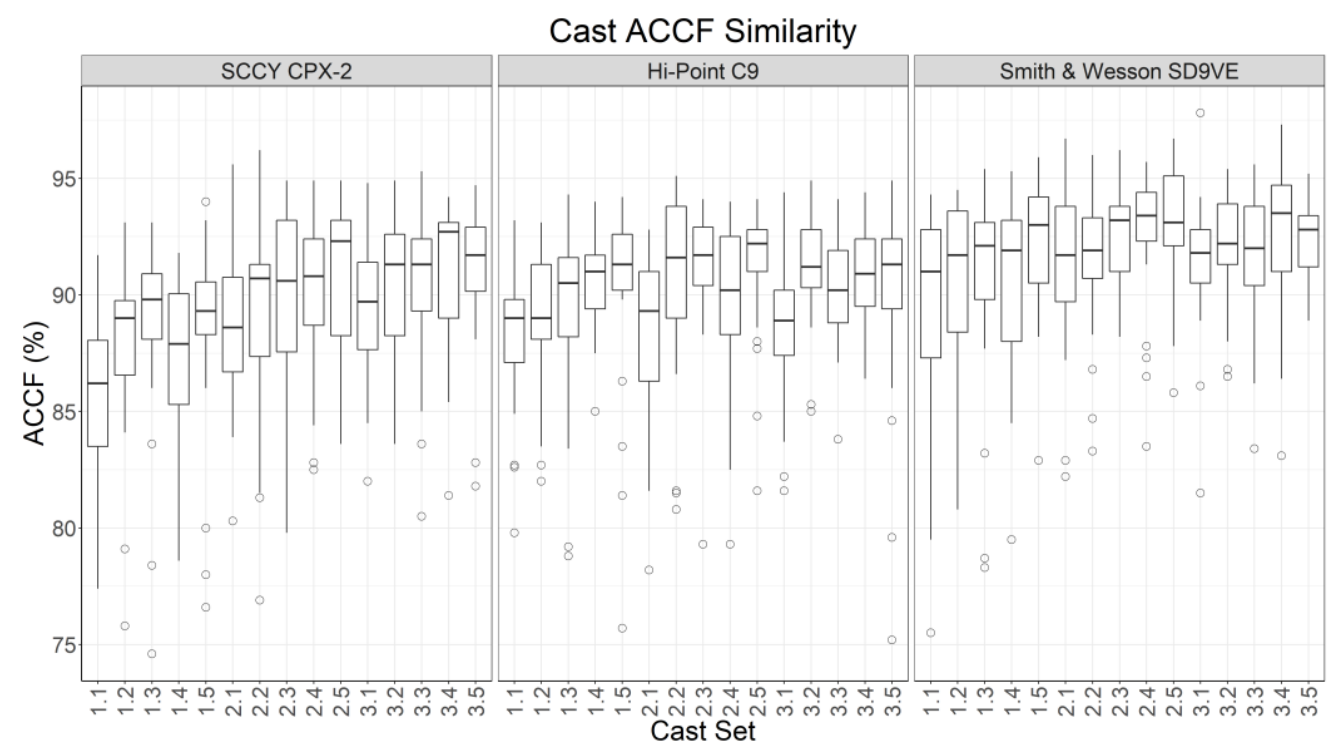

FIG. 7-Boxplot of the ACCF $F_{M A X}$ scores of all casts compared to their master cartridge cases separated by the different mold and set numbers.

Because the Friedman test was significant for the $\mathrm{ACCF}_{\mathrm{MAX}}$ data for all three firearms (Table 2), a multiple comparisons procedure (17) was performed to determine which individual groups were significantly different. Tables 3,4 , and 5 display the $p$-values for the individual comparisons of 
the groups that are displayed in the boxplots with significant differences ( $p$-values less than 0.05) highlighted in gray.

Table 3: Friedman test multiple comparisons procedure for the CPX-2 ACCF Significant differences at the 0.05 level are highlighted in gray.

\begin{tabular}{|l|l|l|l|l|l|l|l|l|l|l|l|l|l|l|l|}
\hline & 1.1 & 1.2 & 1.3 & 1.4 & 1.5 & 2.1 & 2.2 & 2.3 & 2.4 & 2.5 & 3.1 & 3.2 & 3.3 & 3.4 \\
\hline 1.2 & 0.9675 & - & - & - & - & - & - & - & - & - & - & - & - \\
\hline 1.3 & 0.3199 & 0.9987 & - & - & - & - & - & - & - & - & - & - & - \\
\hline 1.4 & 1.0000 & 0.9999 & 0.7826 & - & - & - & - & - & - & - & - & - & - \\
\hline 1.5 & 0.3408 & 0.9991 & 1.0000 & 0.8021 & - & - & - & - & - & - & - & - & - \\
\hline 2.1 & 0.917 & 1.0000 & 0.9999 & 0.9989 & 0.9999 & - & - & - & - & - & - & - & - \\
\hline 2.2 & 0.0158 & 0.6846 & 0.9994 & 0.124 & 0.9991 & 0.8116 & - & - & - & - & - & - & - \\
\hline 2.3 & 0.003 & 0.3734 & 0.9797 & 0.0349 & 0.9754 & 0.5148 & 1.0000 & - & - & - & - & - & - \\
\hline 2.4 & 0.0086 & 0.564 & 0.9969 & 0.0786 & 0.996 & 0.7076 & 1.0000 & 1.0000 & - & - & - & - & - \\
\hline 2.5 & 0.0002 & 0.0992 & 0.7724 & 0.0043 & 0.7516 & 0.1663 & 0.9997 & 1.0000 & 1.0000 & - & - & - & - \\
\hline 3.1 & 0.2899 & 0.998 & 1.0000 & 0.7516 & 1.0000 & 0.9997 & 0.9996 & 0.9851 & 0.998 & 0.8021 & - & - & - \\
\hline 3.2 & 0.0008 & 0.1949 & 0.9051 & 0.0117 & 0.8922 & 0.2997 & 1.0000 & 1.0000 & 1.0000 & 1.0000 & 0.9225 & - & - \\
\hline 3.3 & 0.0002 & 0.0992 & 0.7724 & 0.0043 & 0.7516 & 0.1663 & 0.9997 & 1.0000 & 1.0000 & 1.0000 & 0.8021 & 1.0000 & - \\
\hline 3.4 & $<0.0001$ & 0.033 & 0.5148 & 0.0009 & 0.4904 & 0.0616 & 0.9914 & 0.9999 & 0.9977 & 1.0000 & 0.5517 & 1.0000 & 1.0000 & - \\
\hline 3.5 & $<0.0001$ & 0.0098 & 0.2803 & 0.0002 & 0.2616 & 0.0200 & 0.9376 & 0.9954 & 0.9729 & 1.0000 & 0.3097 & 0.9998 & 1.0000 & 1.0000 \\
\hline
\end{tabular}

Table 4: Friedman test multiple comparisons procedure for the C9 ACCF ${ }_{M A X}$ values. Significant differences at the 0.05 level are highlighted in gray.

\begin{tabular}{|l|l|l|l|l|l|l|l|l|l|l|l|l|l|l|}
\hline & 1.1 & 1.2 & 1.3 & 1.4 & 1.5 & 2.1 & 2.2 & 2.3 & 2.4 & 2.5 & 3.1 & 3.2 & 3.3 & 3.4 \\
\hline 1.2 & 0.9616 & - & - & - & - & - & - & - & - & - & - & - & - & - \\
\hline 1.3 & 0.8832 & 1.0000 & - & - & - & - & - & - & - & - & - & - & - & - \\
\hline 1.4 & 0.1286 & 0.9793 & 0.9965 & - & - & - & - & - & - & - & - & - & - & - \\
\hline 1.5 & 0.0227 & 0.7746 & 0.9025 & 1.0000 & - & - & - & - & - & - & - & - & - & - \\
\hline 2.1 & 0.9998 & 1.0000 & 0.9999 & 0.734 & 0.3283 & - & - & - & - & - & - & - & - & - \\
\hline 2.2 & 0.0038 & 0.4354 & 0.6225 & 0.9995 & 1.0000 & 0.1086 & - & - & - & - & - & - & - & - \\
\hline 2.3 & 0.0026 & 0.3695 & 0.5519 & 0.9987 & 1.0000 & 0.0834 & 1.0000 & - & - & - & - & - & - & - \\
\hline 2.4 & 0.3184 & 0.9991 & 1.0000 & 1.0000 & 0.9998 & 0.9298 & 0.986 & 0.975 & - & - & - & - & - & - \\
\hline 2.5 & 0.0003 & 0.1286 & 0.2368 & 0.9616 & 0.9995 & 0.0182 & 1.0000 & 1.0000 & 0.8121 & - & - & - & - & - \\
\hline 3.1 & 1.0000 & 1.0000 & 0.9992 & 0.5873 & 0.2129 & 1.0000 & 0.0602 & 0.0449 & 0.8461 & 0.0086 & - & - & - & - \\
\hline 3.2 & 0.0053 & 0.493 & 0.6797 & 0.9998 & 1.0000 & 0.134 & 1.0000 & 1.0000 & 0.9919 & 1.0000 & 0.0761 & - & - & - \\
\hline 3.3 & 0.3910 & 0.9997 & 1.0000 & 1.0000 & 0.9993 & 0.9583 & 0.9727 & 0.9549 & 1.0000 & 0.7444 & 0.8963 & 0.9829 & - & - \\
\hline 3.4 & 0.0109 & 0.6341 & 0.8031 & 1.0000 & 1.0000 & 0.2129 & 1.0000 & 1.0000 & 0.9983 & 1.0000 & 0.1286 & 1.0000 & 0.9955 & - \\
\hline 3.5 & 0.0834 & 0.9512 & 0.9887 & 1.0000 & 1.0000 & 0.6225 & 0.9999 & 0.9998 & 1.0000 & 0.9845 & 0.4697 & 1.0000 & 1.0000 & 1.0000 \\
\hline
\end{tabular}


Table 5: Friedman test multiple comparisons procedure for the SD9VE ACCF Significant differences at the 0.05 level are highlighted in gray.

\begin{tabular}{|l|l|l|l|l|l|l|l|l|l|l|l|l|l|l|}
\hline & 1.1 & 1.2 & 1.3 & 1.4 & 1.5 & 2.1 & 2.2 & 2.3 & 2.4 & 2.5 & 3.1 & 3.2 & 3.3 & 3.4 \\
\hline 1.2 & 0.9191 & - & - & - & - & - & - & - & - & - & - & - & - & - \\
\hline 1.3 & 0.7537 & 1.0000 & - & - & - & - & - & - & - & - & - & - & - \\
\hline 1.4 & 0.8533 & 1.0000 & 1.0000 & - & - & - & - & - & - & - & - & - & - & - \\
\hline 1.5 & 0.0791 & 0.9771 & 0.998 & 0.9918 & - & - & - & - & - & - & - & - & - & - \\
\hline 2.1 & 0.8533 & 1.0000 & 1.0000 & 1.0000 & 0.9918 & - & - & - & - & - & - & - & - & - \\
\hline 2.2 & 0.5387 & 1.0000 & 1.0000 & 1.0000 & 0.9999 & 1.0000 & - & - & - & - & - & - & - & - \\
\hline 2.3 & 0.0345 & 0.9136 & 0.9844 & 0.9581 & 1.0000 & 0.9581 & 0.9985 & - & - & - & - & - & - & - \\
\hline 2.4 & 0.0011 & 0.3371 & 0.5742 & 0.4454 & 0.9983 & 0.4454 & 0.7834 & 0.9999 & - & - & - & - & - & - \\
\hline 2.5 & 0.0009 & 0.3074 & 0.5387 & 0.4117 & 0.9973 & 0.4117 & 0.7537 & 0.9998 & 1.0000 & - & - & - & - & - \\
\hline 3.1 & 0.9942 & 1.0000 & 1.0000 & 1.0000 & 0.8288 & 1.0000 & 0.9992 & 0.6559 & 0.1176 & 0.1034 & - & - & - & - \\
\hline 3.2 & 0.6559 & 1.0000 & 1.0000 & 1.0000 & 0.9995 & 1.0000 & 1.0000 & 0.9942 & 0.6785 & 0.6444 & 0.9999 & - & - & - \\
\hline 3.3 & 0.9918 & 1.0000 & 1.0000 & 1.0000 & 0.8533 & 1.0000 & 0.9995 & 0.6897 & 0.1332 & 0.1176 & 1.0000 & 0.9999 & - & - \\
\hline 3.4 & 0.0071 & 0.6673 & 0.8684 & 0.7737 & 1.0000 & 0.7737 & 0.9644 & 1.0000 & 1.0000 & 1.0000 & 0.3371 & 0.9244 & 0.3682 & - \\
\hline 3.5 & 0.0721 & 0.9725 & 0.9973 & 0.9898 & 1.0000 & 0.9898 & 0.9999 & 1.0000 & 0.9987 & 0.998 & 0.8113 & 0.9993 & 0.8372 & 1.0000 \\
\hline
\end{tabular}

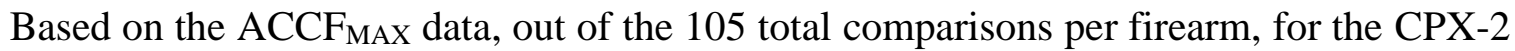
there were 17 groups that were significantly different, for the $\mathrm{C} 9$ there were nine groups that were significantly different, and for the SD9VE there were four groups that were significantly different. Out of these significant differences, only two were within-mold differences (Cast Sets 1.1 to 1.5 and 2.1 to 2.5 with the C9). All other differences were between-mold comparisons. This indicates that within-mold variability is minimal, and between-mold variability is low.

Previous 2D IBIS $®$ breech face results showed more differences between the groups. There were 42 group differences for the CPX-2, 51 for the C9, and 50 for the SD9VE (7). This was mainly due to reproductions made from Mold 1 which accounted for 40 of the 42 differences for the CPX-2, 50 of the 51 differences for the C9, and all 50 for the SD9VE (7). While not as many differences with Mold 1 were evident using the 3D data, out of the 30 differences discovered across all three firearms, only four did not involve Mold 1 comparisons. This indicates there is still something about Mold 1 double-casts that is causing some differences in automated comparisons. However, for the 3D data Mold 1 did not visually produce larger scores as was evident when examining the boxplots of the 2D IBIS $®$ data (7). 
There is some contradiction in which groups are significantly different depending on which data are used (2D or 3D). Consider row 2.1 in Table 5 as an example. All $p$-values are nonsignificant. Furthermore, three of the five values are equal to 1.0000 , and the minimum value is 0.8533. Examining these same comparisons based on the IBIS ${ }^{\circledR}$ correlation results, all the $p$-values were less than 0.0001 (7). The discrepancies here represent the two extremes: with the IBIS@ data there is abundant evidence of a difference between the groups, while with the 3D data there is almost no evidence of any difference. The reverse is also true in some instances. For example, refer to the comparison of 2.1 to 2.5 for the C9 in Table 4 . The $p$-value for the 3D data was 0.0182 , a significant result. However, the $p$-value for the same comparison based on the IBIS ${ }^{\circledR}$ data was 1.0000 (7). Again, this represents two opposite conclusions depending on which data are used. The comparison between 2.5 and 3.1 for the $\mathrm{C} 9$ is a similar case, and others can be found for the other two firearms. The cause of this difference needs to be explored in further research.

Table 6 summarizes how the significant and nonsignificant results between the 2D (7) and 3D similarity scores compare. The significant and nonsignificant columns represent comparisons where both the 2D and 3D data were significant and nonsignificant, respectively. There is also a contradict column. These were comparisons where the interpretation (significant or nonsignificant) changed depending on which data were used. In these instances where the results were contradictory, the results were significant for the 3D data in two CPX-2 comparisons, four C9 comparisons, and zero SD9VE comparisons.

Table 6: Comparison of significant results between $2 D(7)$ and $3 D$ data.

\begin{tabular}{|l|l|l|l|}
\hline Firearm & Significant & Nonsignificant & Contradict \\
\hline SCCY CPX-2 & 15 & 61 & 29 \\
\hline Hi-Point C9 & 5 & 50 & 50 \\
\hline Smith \& Wesson SD9VE & 4 & 55 & 46 \\
\hline
\end{tabular}

Figs. 8, 9, and 10 each display a master cartridge case and reproduction that resulted in one of the lowest overall similarity values from one of the three firearms. Due to the allowable thresholds for $\mathrm{CMC}$, the low $\mathrm{ACCF}_{\mathrm{MAX}}$ comparisons were still able to produce a large number of congruent cells: 37 of 39 for the CPX-2, 34 of 36 for the C9, and 39 of 39 for the SD9VE. Although 
some differences may be found, the reproductions are still visually accurate representations of the features from the master cartridge cases.
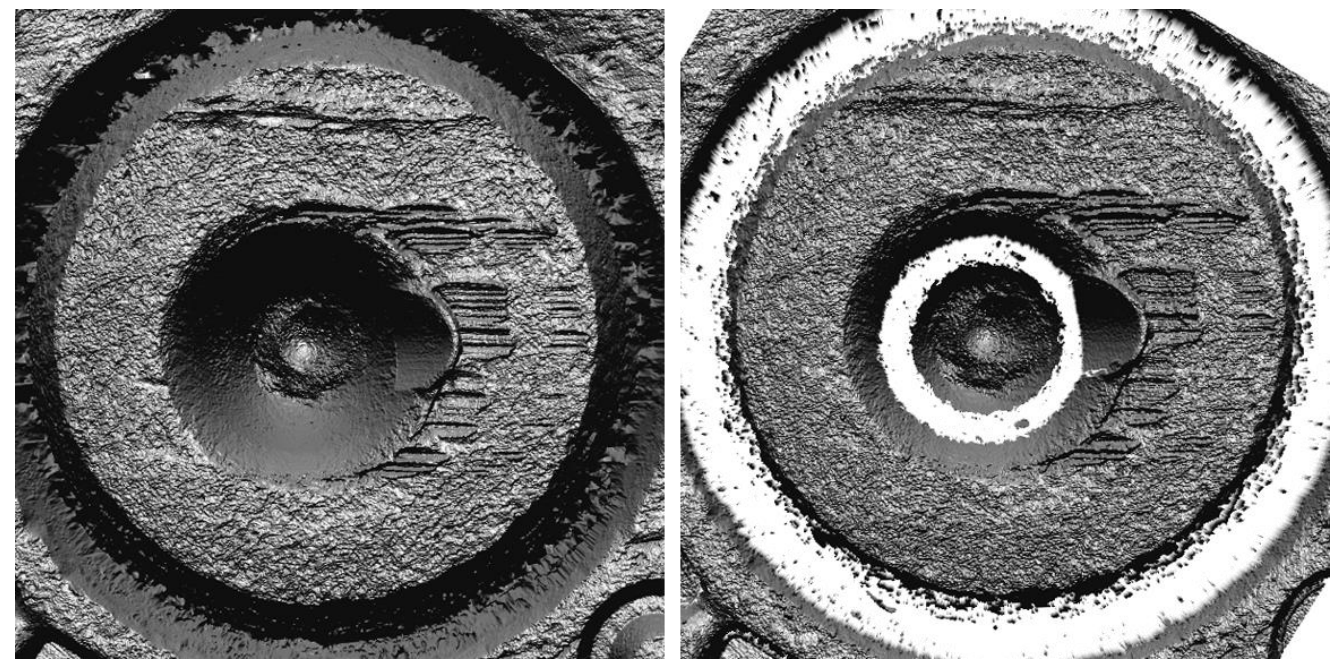

FIG. 8-One of the lowest ACCF $F_{M A X}$ comparisons for the CPX-2, resulting in an ACCF $F_{M A X}$ of $76.6 \%$ and 37 of 39 congruent cells. The master cartridge case is on the left and the reproduction from Mold 1, Set 5 is on the right. Images saved from the Cadre X3P viewer (8) with the enhanced contrast option enabled.
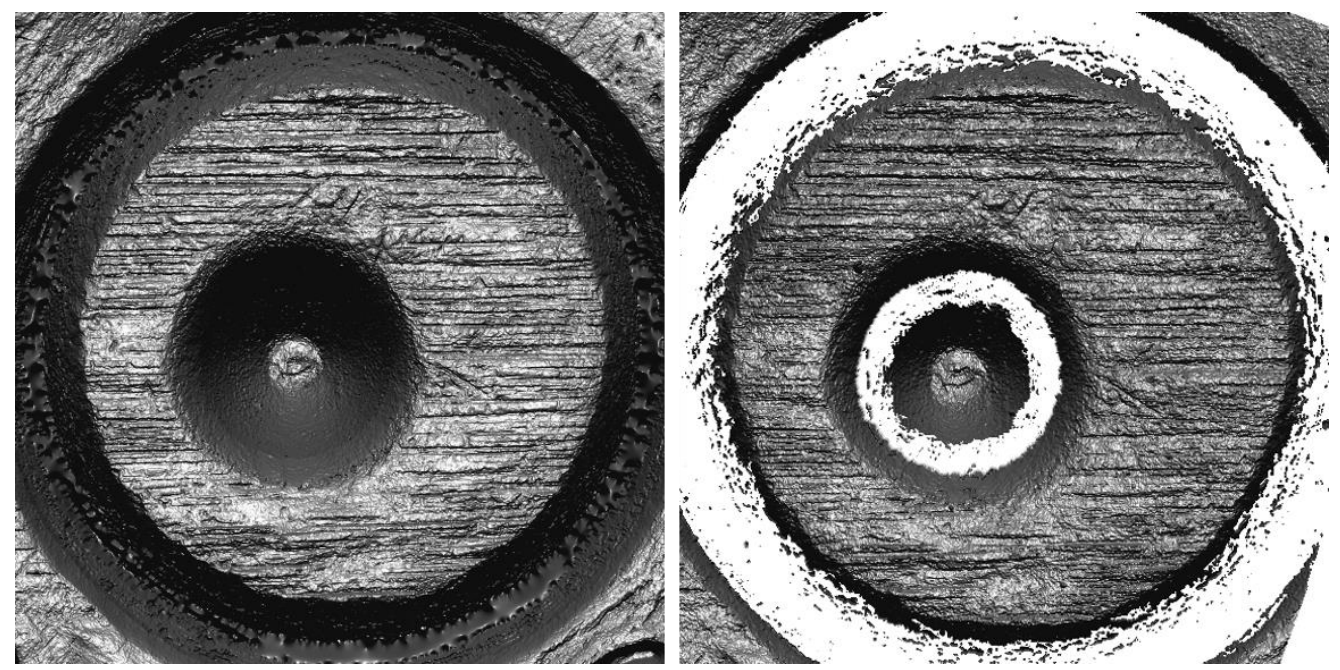

FIG. 9-One of the lowest ACCF $F_{M A X}$ comparisons for the C9, resulting in an ACCF $F_{M A X}$ of $78.8 \%$ and 34 of 35 congruent cells. The master cartridge case is on the left and the reproduction from Mold 1, Set 3 is on the right. Images saved from the Cadre X3P viewer (8) with the enhanced contrast option enabled. 

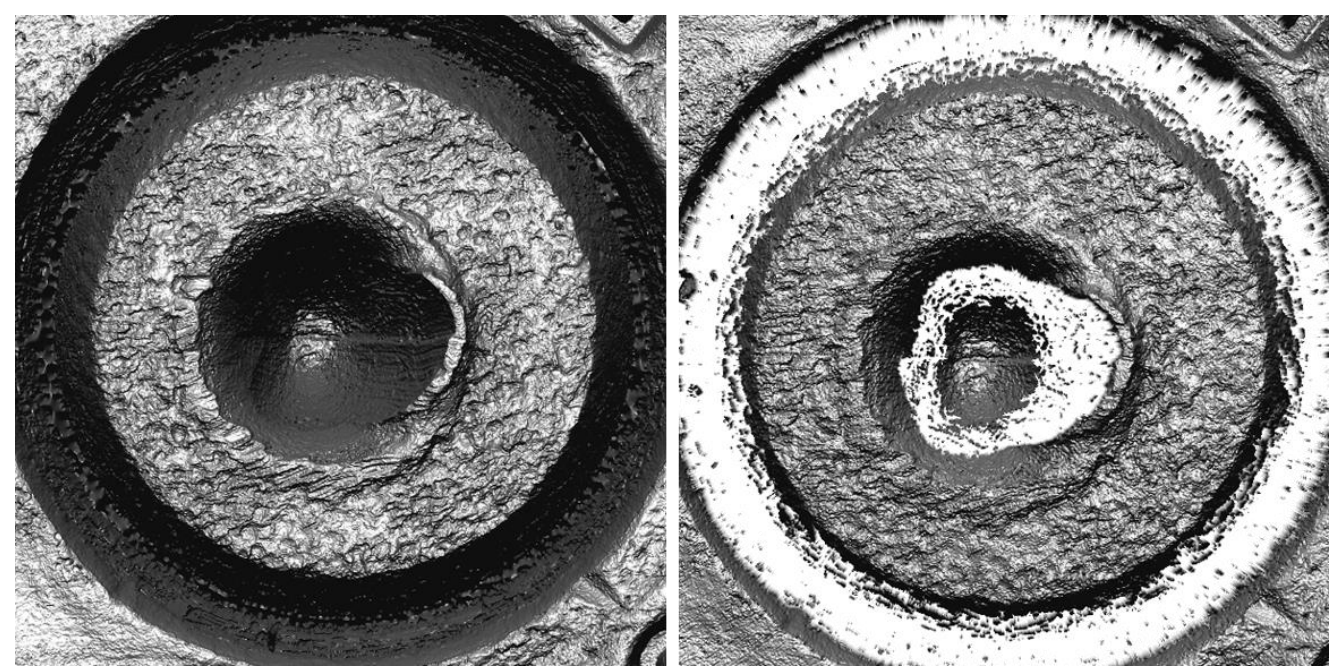

FIG. 10-One of the lowest ACCF ${ }_{M A X}$ comparisons for the SD9VE, resulting in an ACCF ${ }_{M A X}$ of $75.5 \%$ and 39 of 39 congruent cells. The master cartridge case is on the left and the reproduction from Mold 1, Set 1 is on the right. Images saved from the Cadre X3P viewer (8) with the enhanced contrast option enabled.

\section{CMC Data}

The $\mathrm{ACCF}_{\mathrm{MAX}}$ may be the more appropriate metric to use for comparing the accuracy of double-casting because the overall similarity is of interest rather than individual areas covered by cells. However, the congruent cell data is also important because many comparisons not involving reproductions to their master cartridge cases will not result in $100 \%$ of potential cells being congruent due to shot to shot variability. Refer to Fig. 3 for a typical known match example.

As discussed earlier, the CMC data were presented as a percent value by dividing the number of congruent cells by the total number of potential cells for each comparison. Recall, the number of potential cells were overlaid onto the reference surface (the master cartridge case) and searched over the comparison surface (the double-cast). Any cells that were within the similarity, positioning, and angular thresholds were considered congruent. The distributions of the percentages of congruent cells are displayed in Fig. 11. For the CPX-2, $62 \%$ of comparisons resulted in $100 \%$ congruent cells, $83 \%$ were $100 \%$ congruent for the C9, and $82 \%$ were $100 \%$ congruent for the SD9VE. 


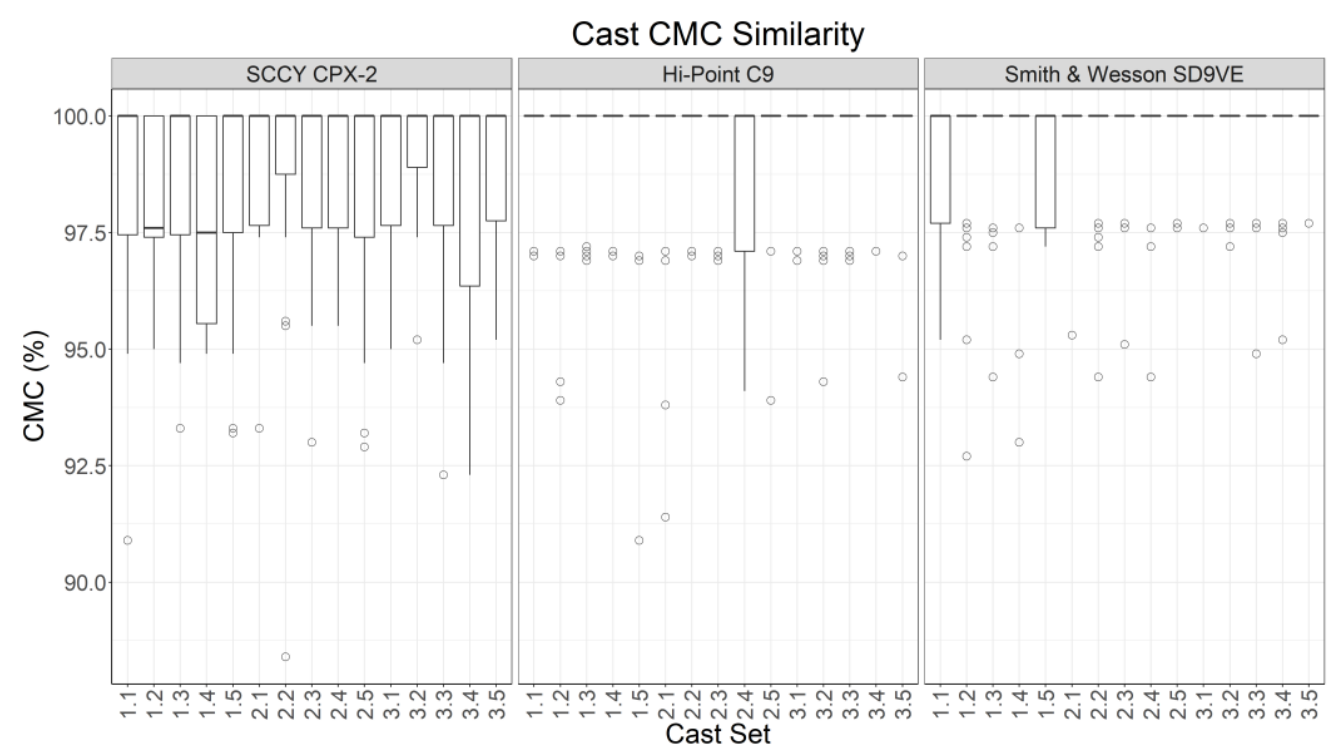

FIG. 11-Boxplot of the CMC percent values of all casts compared to their master cartridge cases separated by the different mold and set numbers.

The Friedman test results for the congruent cell data were significant for the CPX-2 and SD9VE, so a multiple comparisons procedure was used for those two firearms to determine which comparisons were significant. The minimum $p$-values for the CPX-2 and SD9VE were 0.0850 and 0.1500 , respectively, indicating no individual significant differences, and therefore the $p$-values are not all being included here as with the $\mathrm{ACCF}_{\mathrm{MAX}}$ data. There may have been no individual significant differences found with the multiple comparisons test for a couple of reasons. The Friedman test $p$-values were not much below the significance level of 0.05 . Because of this, the multiple comparisons procedure may not have had enough statistical power to detect any individual group differences at those Friedman test $p$-value magnitudes.

Double-casts of cartridge cases from the CPX-2 seemed to produce lower overall

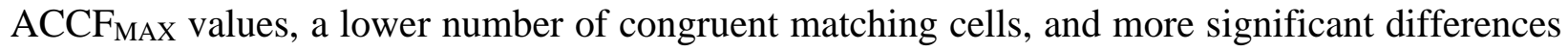
( $p$-values less than 0.05). Further research is required to determine why the CPX-2 showed this difference compared with the other two firearms.

\section{Conclusions}

Three-dimensional analysis utilizing the $\mathrm{ACCF}_{\mathrm{MAX}}$ and $\mathrm{CMC}$ data shows that the doublecasting process creates reproductions that are representative of the fine detail present in the surfaces of the master cartridge cases. The double-casts from this study with the lowest overall 
similarity percentages still visually produced the detail from the master cartridge cases. Furthermore, implementation of a quality control process would lead to removal of any reproductions that are below the desired quality, whether measured through objective or visual comparisons. Double-casting has importance in the forensic science community for easily creating standards for database imaging, proficiency testing, and error rate analysis. Based on the results presented, double-cast sets could be created for these purposes with little concern for being inaccurate cartridge case representations.

\section{References}

1. Song J, Whitenton E, Kelley D, Clary R, Ma L, Ballou S, Ols M, SRM 2460/2461 standard bullets and casings project, J Res Natl Inst Stand Technol 2004;109(6):533-542. doi: 10.6028/jres.109.040.

2. Seiler DF and Watters Jr RL, NIST Certificate: Standard Reference Material 2461 Standard Cartridge Case, 2012.

3. Biasotti AA, Plastic replicas in firearms and tool mark identifications, J Crim Law Criminol 1956;47(1):110-117.

4. General Secretariat, Firearms Programme, INTERPOL Ballistics Information Network: Handbook on the Collection and Sharing of Ballistics Data, INTERPOL, Lyon, France, 3rd edition, 2014.

5. Pauw-Vugts P, Walters A, Øren L, Pfoser L, Faid2009: Proficiency test and workshop, AFTE J 2013;45(2):115-127.

6. Gundlach D, Choquette SJ, NIST Certificate: Standard Reference Material 2460a Standard Bullet Replica, NIST, 2018.

7. Law E, Morris K, The utility of double-casting for creating cartridge case reproductions, AFTE J 2020;52(1):26-39.

8. Cadre Forensics, X3P Viewer Software. Available by request at www.cadreforensics.com as of August 15, 2019.

9. Open Forensic Metrology Consortium, OpenFMC \& X3P. Available at

https://www.cadreforensics.com/x3p.html as of August 21, 2019.

10. Song J, Proposed NIST ballistics identification system (NBIS) using 3D topography measurements on correlation cells, AFTE J 2013;45(2):184-189. 
11. Chu W, Tong M, Song J, Validation tests for the congruent matching cells (CMC) method using cartridge cases fired with consecutively manufactured pistol slides, AFTE J 2013;45(4):361366.

12. Song J, Vorburger TV, Chu W, Yen J, Soons JA, Ott DB, et al.,

Estimating error rates for firearm evidence identifications in forensic science, Forensic Sci Int 2018;284:15-32. doi: 10.1016/j.forsciint.2017.12.013.

13. R Core Team, R: A Language and Environment for Statistical Computing, R Foundation for Statistical Computing, Vienna, Austria, 2019.

14. RStudio, Inc, About RStudio. Retrieved from http://www.rstudio.com/about, 2019.

15. Smooth-Cast ${ }^{\mathrm{TM}}$ ColorMatch $^{\mathrm{TM}} 325$ Series, Technical bulletin. Available at https://www.smooth-on.com/tb/files/SMOOTHCAST_325_326_327_COMBO_TB.pdf as of 06May-2020.

16. Conover WJ, Practical Nonparametric Statistics, Wiley Series in Probability and Mathematical Statistics, John Wiley and Sons, 2nd edition, 1980. ISBN13: 9780471028673.

17. Pohlert T, PMCMRplus: Calculate Pairwise Multiple Comparisons of Mean Rank Sums Extended, 2018. R package version 1.4.1. 


\title{
4. Evaluating firearm examiner conclusion variability using cartridge case reproductions
}

\section{Publication status}

This chapter is in final preparations for submission to a forensic science journal.

\begin{abstract}
The forensic science pattern comparison areas, including fingerprints, footwear, and firearms, have been criticized for their subjective nature. While much research has attempted to move these disciplines to more objective methods, examiners are still coming to conclusions based on their own training and experience. To complement this subjectivity, black box studies are necessary to establish the accuracy of these feature comparison methods. However, when cartridges are fired by a firearm to create cartridge case test sets there may be significant variability within the resulting impressions. This can result in different participants receiving test sets with varying levels of difficulty based on differences in impression quality. Therefore, comparison of accuracy between examiners is not straightforward. To compare accuracy between examiners, a method called double-casting was used to create plastic cartridge case reproductions. Double-casts of twenty-one test sets of master cartridge cases were created and mailed to firearm examiners. The double-casts ensured that all participants were comparing exhibits with the same level of detail. The examiners were tasked with determining if the unknown cartridge case in each set was fired by the same firearm as the three knowns. Automated comparisons were also used to compare the cartridge cases within each set. The results from this study showed that there are differences in examiner conclusions when examining the same evidence. Furthermore, it was shown that automated comparison metrics would benefit examiners as a quality control measure to correct any potential errors and strengthen conclusions.
\end{abstract}




\section{Introduction}

One concern that is continually raised in reference to the pattern matching disciplines of forensic science, such as firearms examination, is that conclusions are based solely on the opinion of the examiner analyzing the evidence in a case $[1,2]$. This subjectivity frequently has a negative connotation in forensic science. To understand the strength of reported conclusions, error rates need to be assessed.

Many previous error rate studies have focused on the use of consecutively manufactured surfaces [3-6]. The goal was to determine the ability of examiners to separate matching and nonmatching cartridge cases under the worst-case scenario because consecutively machined surfaces are those that are likely to be most similar. Others had the goal of presenting more realistic samples to participants, either from firearms that had been submitted in casework or were commonly available and/or carried by officers in the jurisdiction of the author(s) [7-10]. Generally these studies have demonstrated low error rates in cartridge case comparisons. To further understand error rates, the question "will firearm examiners come to the same conclusions when comparing the same cartridge cases", also known as reproducibility, is important. To analyze reproducibility, sets of cartridge cases fired by the same firearm sent to multiple examiners cannot be used because cartridge cases have inherent variability in their breech face and firing pin impression areas even when fired by the same firearm.

As an example of the impact of cartridge case variability on comparisons, suppose a researcher decides to create a comparison set of fired cartridge cases to test the ability of examiners to reach the correct conclusion. The researcher creates two test sets of four cartridge cases, each with one questioned and three knowns, by firing eight shots of the same ammunition from a single firearm. Two examiners are recruited for the study, Examiner A and Examiner B. The examiners are asked to determine if the questioned cartridge case was fired by the same firearm as the three knowns. The questioned cartridge case received by Examiner A is shown in Figure 1 (left) and the questioned cartridge case received by Examiner B is shown in Figure 1 (right). Figure 1 (left) has little to no aperture shearing, while Figure 1 (right) has much more aperture shearing present for comparison. This example is a simple one, yet it summarizes the effect cartridge case variability may have on the comparison process. Due to differences in comparison difficulty based on the level of detail present, there is the potential for examiners to come to different conclusions.

To establish the foundational validity of a scientific method, it must be repeatable, reproducible, and accurate [2]. While previous error rate studies have focused on addressing the accuracy portion of that definition, studies assessing repeatability and reproducibility have been lacking. Therefore, to further understand foundational validity in cartridge case comparisons, it is important to analyze conclusions by experts that have observed the same evidence. Plastic reproductions of cartridge 


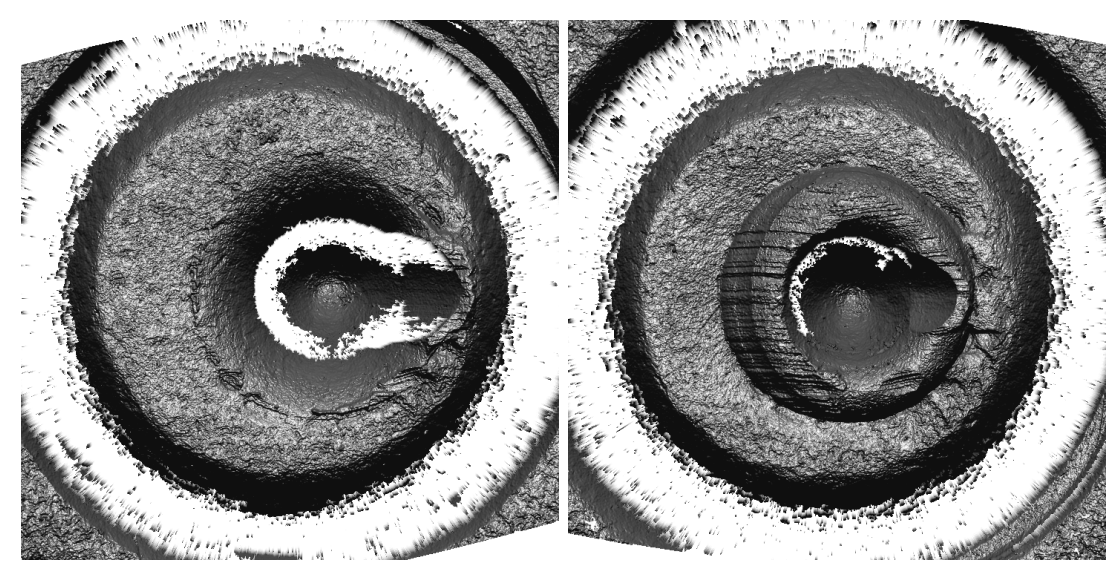

Figure 1: Two successively fired $9 \mathrm{~mm}$ Luger cartridge cases from the same Ruger SR9. Images were saved using the Cadre X3P Viewer software [11].

cases, called double-casts, have been used in a proficiency test to ensure all laboratories were examining the same evidence [12]. A validation study on virtual comparison microscopy (VCM) eliminated test variability by using three-dimensional (3D) cartridge case scans, and therefore participants were examining the same cartridge cases [13]. Examiner variability has been further analyzed by providing participants with the same two-dimensional (2D) comparison images of Glock aperture shearing [14]. These studies have shown that examiners generally come to the same conclusions when analyzing the same evidence.

To assess reproducibility among the conclusions of examiners using physical samples and to allow examiners to perform comparisons as they would in casework, double-casting was used in this study to replicate sets of fired cartridge cases to send to participants. The double-casting method employed here has been shown to produce accurate reproductions $[15,16]$. In this way, all participants performed comparisons on items with the same amount of information present in each item, although the comparisons themselves were completed using the reproductions.

\section{Methods}

\section{Examiner test sets}

For a study examining error rates in cartridge case comparisons, it is important to select a group of firearms that are representative of what firearm examiners encounter in casework, with some that reproduce markings well and some that do not. The firearms selected for this study have been shown to produce a range of performance based on analysis of similarity scores from a legacy 2D Integrated Ballistics Identification System (IBIS) [17]. In total, 21 sets of cartridge cases were presented to each participant. The first 20 sets included three cartridge cases representing test fires 
from a particular firearm with an additional questioned cartridge case for comparison. Table 1 displays the firearms used to provide the three cartridge cases as test fires for each test set, with each set using a unique sample firearm (i.e. no sample firearms were repeated). Seven were same-source and 13 were different-source. The number of same-source and different-source sets was different to prevent examiners from assuming they were equal and using that to assist in their conclusions. Furthermore, false positives are viewed as a larger problem than false negatives, and for that reason there were more different-source sets. For the known different-source sets, the same model firearm was used for the known and questioned cartridge cases to present close non-matching comparisons. This prevented eliminations from being made based on class characteristics alone. If this had not been the case, the results of the study would not be meaningful, as it is assumed that firearm examiners would be able to separate cartridge cases fired from firearms with different class characteristics. One different-source set included a Ruger P95 for the known cartridge cases and a Ruger SR9 for the questioned cartridge case (Set 7 in Table 1). Although the firearms are different models, they share similar class characteristics. An additional test set, Set 21, was designed that consisted of ten fired cartridge cases shown in Table 2. The examiners were asked to report the number of firearms used to compose that set.

Table 1: Firearms used to test fire for the known cartridge cases for Sets 1-20. All firearms are chambered in $9 \mathrm{~mm}$ Luger.

\begin{tabular}{lll}
\hline Set Number & Known Firearm & Ground Truth \\
\hline 1 & Taurus 24/7 G2 & Elimination \\
2 & SCCY CPX-2 & Elimination \\
3 & Springfield XD9 & Identification \\
4 & Ruger LC9 & Elimination \\
5 & Hi-Point C9 & Elimination \\
6 & Keltec PF9 & Identification \\
7 & Ruger P95 & Elimination \\
8 & Ruger LC9 & Elimination \\
9 & SCCY CPX-2 & Elimination \\
10 & Hi-Point C9 & Identification \\
11 & Smith \& Wesson SD9VE & Identification \\
12 & Springfield XD9 & Elimination \\
13 & Hi-Point C9 & Elimination \\
14 & Hi-Point C9 & Elimination \\
15 & SCCY CPX-2 & Elimination \\
16 & Taurus 24/7 G2 & Elimination \\
17 & Ruger LC9 & Identification \\
18 & Taurus 24/7 G2 & Identification \\
19 & Springfield XD9 & Elimination \\
20 & SCCY CPX-2 & Identification \\
\hline
\end{tabular}


Table 2: Set 21 composition related to the number of firearms. All firearms are chambered in $9 \mathrm{~mm}$ Luger.

\begin{tabular}{llll}
\hline $\begin{array}{l}\text { Number of } \\
\text { Cartridge Cases }\end{array}$ & Firearm & Identifier & Cartridge Cases \\
\hline 1 & Ruger LC9 & X32446 & DD \\
3 & Ruger LC9 & X43521 & LI, MX, UB \\
2 & SCCY CPX-2 & X97569 & AQ, KB \\
1 & SCCY CPX-2 & X97568 & TY \\
3 & Taurus 24/7 G2 & X55720 & NB, XS, RT \\
\hline
\end{tabular}

Firearm examiners typically perform test fires using the same type of ammunition as the questioned cartridge case if possible, and for this reason only one type of ammunition was used for the master cartridge cases. The ammunition for all shots was Federal American Eagle with 124 grain full metal jacket bullets. All of the test fires were performed on the same day and collected in plastic bags that identified the cartridge cases to the gun that fired them. Each firearm was assigned a letter and each cartridge case was marked with a numbered sticky label. The label included a letter for the firearm and a number to identify the cartridge case (the cases were not collected in a way that the order of firing could be determined). All test fired cartridge cases were examined under a Leica FSC comparison microscope and imaged with an attached Nikon D5200. Cartridge cases were then selected for the test sets, and the numbered labels on those selected were replaced with a random two letter string that was engraved on each cartridge case. These engravings were transferred to each reproduction through the molding and casting processes. A key was then created to trace each cartridge case back to its original image, set number, and the firearm from which it was fired.

Prior to double-casting, the test sets were all mailed to a practicing firearm examiner. The examiner went though each cartridge case within each test set to analyze the markings and provide a difficulty rating to each set. The goal here was to ensure that the test sets covered a range of difficulty levels. Once this had been established, double-casts were created of all test sets following a previously established procedure $[15,16]$. Figure 2 displays a double-cast and its master cartridge case as an example of a double-cast used in the test sets (K1 from Set 9).

Prior to receiving any comparison sets, each of the 18 examiners that volunteered for this study were given a random alphanumeric string for confidentiality of responses and asked to fill out a short background and experience survey. The results for the background questions and cartridge case comparisons were submitted using an online form through Qualtrics [18]. In this way, receiving the response data was quicker than by mail, and the electronic responses allowed for faster analysis of the data by removing the step of having to enter typed responses into a computer (elim- 


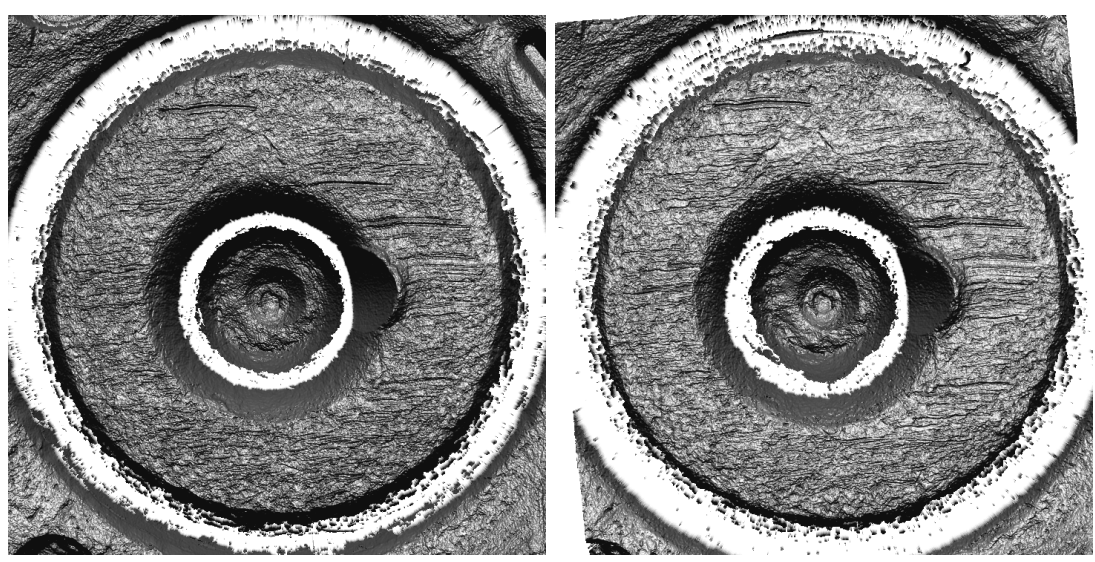

Figure 2: Original cartridge case fired from a SCCY CPX-2 (left) and double-cast (right). Images were saved using the Cadre X3P Viewer software [11].

inating transcription errors). The participants were all asked to perform the comparisons as they would in routine casework.

This was an open-set study where no indications were given to the participants about the number of ground truth identification and elimination sets. Furthermore, each set was independent in the sense that the answer to one set in no way influenced the answers to subsequent sets. This is a West Virginia University (WVU) Institutional Review Board (IRB) approved study (protocol \#1812383090).

\section{Inconclusive conclusions}

The examiners were asked to follow the Association of Firearm and Toolmark Examiners (AFTE) Range of Conclusions [19]. One of the three options is "Inconclusive", however, inconclusive results can be misleading when evaluating accuracy of examiner conclusions. Take ten comparison sets by an examiner as an example. If the correct ground truth conclusion is made in eight of the ten sets, but the other two sets are inconclusive, then a $100 \%$ accuracy is achieved if inconclusives are removed or considered as correct. Imagine a second examiner does the same ten comparison sets and makes the correct ground truth conclusion on all ten sets, also leading to a $100 \%$ accuracy. The accuracy percentages are the same for both examiners, but their performances are not necessarily equal. The first examiner is reducing the chance of errors (false positives and false negatives) by making inconclusive conclusions but at the same time increasing the number of missed identifications and exclusions [20]. In previous research on examiner accuracy, inconclusive conclusions have been considered in different ways. Some studies have not considered inconclusives as incorrect $[4,7,8,12,13,21,22]$, some have considered them separately $[6,9,10,14,23]$, and others did not address inconclusives [3, 5, 24]. 
To obtain more information from inconclusive conclusions, the Qualtrics response form designed for this study asked participants to indicate which of the three subcategories of inconclusive, as specified in the AFTE Range of Conclusions, the comparison resulted. The subcategories are defined by AFTE as [19]:

- Inconclusive A: Some agreement of individual characteristics and all discernible class characteristics, but insufficient for an identification.

- Inconclusive B: Agreement of all discernible class characteristics without agreement or disagreement of individual characteristics due to an absence, insufficiency, or lack of reproducibility.

- Inconclusive C: Agreement of all discernible class characteristics and disagreement of individual characteristics, but insufficient for an elimination.

In this study, subcategory B was considered a "true" inconclusive, where the examiner was not sure one way or another whether the comparison was an identification or elimination. Subcategory A fit the conclusions where the examiner was leaning towards making an identification, but not quite enough similarities were present to make an identification. Similarly with subcategory $\mathrm{C}$, the examiner was leaning towards making an elimination, but there were not enough dissimilarities to make an elimination. It is important to consider these levels of inconclusive because there is some evidential value to subcategory $\mathrm{A}$ and $\mathrm{C}$ responses. If all of these are considered together as simply "inconclusive" then information is being lost in those conclusions [25].

One way to analyze whether there is sufficient information in a comparison to come to the ground truth conclusion is to measure consensus. The Scientific Working Group on Friction Ridge Analysis, Study, and Technology (SWGFAST) says that inconclusive conclusions cannot be correct or incorrect but can only be considered non-consensus [20]. In this way, errors can be determined based on non-consensus inconclusives. The Scientific Working Group for Firearms and Toolmarks (SWGGUN) does not have any documents with recommendations on the evaluation of inconclusive conclusions. To determine consensus conclusions, boxplots were created and the interquartile ranges (IQR) for each set were calculated. The IQR for each set was based on the inconclusive subcategory responses. Any conclusions that fell within the IQR were considered as acceptable conclusions, even if they were not the correct ground truth conclusion. As an overall performance metric, the Cochran test was used to compare between examiners [26].

\section{Automated comparisons}

All test set master cartridge cases were scanned on a Sensofar S neox 3D optical profiler using the "Confocal Fusion" scan mode. This proprietary algorithm developed by Sensofar combines 
confocal microscopy with focus variation. If a confocal data point is measured at a given location then it is recorded. If a confocal measurement is unsuccessful at a given location (e.g. areas with steep slopes) then focus variation measurements are recorded instead. Generally this method works well to combine the strengths of both techniques [27], however, some areas sloping down into the firing pin impression are still difficult to illuminate and measure while not oversaturating the breech face. These areas are not too concerning, as they are not typically used in the comparison process. For consistency purposes, orientation was set with the drag mark in the 3 o 'clock position if present, or parallel impressions and striations running from left to right. All scanned cartridge cases were converted to the *.x3p file format for software interchangeability [28].

The scanned surfaces were then separated into their respective comparison sets. The National Institute of Standards and Technology (NIST) automated comparison software was used, through an on-going collaboration, to inter-compare the three knowns, as well as compare the unknown to each of the three knowns for each comparison set [29-31]. To pre-process the surfaces prior to comparison, the surfaces were trimmed to include only the breech face. This included removal of flowback around the firing pin impression. A Gaussian regression filter with cutoff wavelengths of $25 \mu \mathrm{m}$ and $400 \mu \mathrm{m}$ were used to attenuate noise, form, and waviness. The lateral resolution of each surface was downsampled from $1.38 \mu \mathrm{m}$ per pixel to $2.76 \mu \mathrm{m}$ per pixel to reduce comparison time without decreasing accuracy. Two similarity metrics were applied: the areal correlation coefficient (ACCF) and number of congruent matching cells (CMC). The ACCF corresponds to the overall surface similarity, while the CMC algorithm is based on discretization of the cartridge case surfaces into smaller cells used for comparison. Using the CMC metric, a grid of $500 \mu \mathrm{m} \times 500 \mu \mathrm{m}$ cells was defined on one surface. For each of the cells, a search was performed over all positions and $360^{\circ}$ in rotation on the comparison surface to find the location on the comparison surface which yielded the highest similarity value. This was repeated for each of the cells. The number of congruent cells metric is defined by the number of cell pairs that have a sufficient similarity and a congruent registration location. In this study to be considered congruent, the cell similarity value had to be at least $20 \%$ and the errors in registration position and orientation were not allowed to exceed $125 \mu \mathrm{m}$ and $3^{\circ}$, respectively.

Ten additional cartridge cases from each of the 26 firearms used throughout this study were collected, scanned, and compared using the same method as described above. These cartridge cases were used to provide additional information about the performance of each firearm. It is important to note here that examiners were not provided with the automated comparison results to consider during their comparisons. 


\section{Automated comparison interpretation}

Interpretation of automated comparison results has some subjectivity. To understand how these data are interpreted, five students in the WVU Department of Forensic and Investigative Science were shown the algorithm results for the comparison sets that were sent to examiners. Students were used for this portion of the study because these NIST algorithms are research-based and not currently in use by practicing firearm examiners in crime laboratories. These students each had differing levels of experience with interpretation of automated comparison results in the pattern comparison areas of forensic science, and at least some knowledge of the ACCF and CMC algorithms in particular. The students were not shown the physical cartridge cases or images of the cartridge cases. They were simply asked to state their conclusion for each set, using the AFTE Range of Conclusions, based on only the ACCF and CMC metrics.

There were two parts to this test. Part 1 included ACCF and CMC results of comparisons from only the cartridge cases that were sent to examiners. Therefore, three comparisons were shown for the three known cartridge cases ( $\mathrm{K} 1$ to $\mathrm{K} 2, \mathrm{~K} 1$ to $\mathrm{K} 3$, and $\mathrm{K} 2$ to $\mathrm{K} 3$ ) and three comparisons were shown for the questioned cartridge case ( $\mathrm{Q}$ to $\mathrm{K} 1, \mathrm{Q}$ to $\mathrm{K} 2$, and $\mathrm{Q}$ to K3). Part 2 consisted of all test set results from Part 1 , as well as the automated comparison results from the additional ten cartridge cases collected from each firearm. While sample firearm performance would not typically be available in a case, the results were included to determine what effect, if any, additional information on sample firearm performance had on the students' conclusions compared to Part 1. Receiver operating characteristic (ROC) area under the curve (AUC) values were also calculated and provided to the students in Part 2 [32]. This portion of the study was approved through the WVU IRB (protocol \#2004971018).

\section{Results and Discussion}

\section{Examiner comparisons - ground truth analysis}

Prior to discussing the results, a note must be made in reference to one of the participants. This participant completed the first 20 comparison sets and then decided to withdraw from the study. However, five false positive conclusions were made by this participant on Sets 2, 7, 9, 14, and 16. These data are being included here for completeness, but, due to this participant's decision to withdraw, will not be included in the remainder of the analysis. This is in accordance with federal research guidelines [33]. The results of this study have not been shared or discussed with any participants, including the participant that chose to withdraw.

Participant conclusions for the comparison sets are displayed in Figure 3. For all inconclusive responses during data collection, participants were requested to select the appropriate subcategory 
based on the AFTE Range of Conclusions [19]. This provided more detailed information about each inconclusive conclusion. All participants came to the same, correct conclusion for Sets 3, 6,10 , and 11 which was an identification. For the remaining three ground truth identifications there was some variability within the conclusions. For all of the 13 ground truth elimination comparisons, there were no sets where all participants came to the same conclusion. There was one false positive conclusion in the study on Set 16 (participant XZD8SWR with a Taurus 24/7 G2 pistol). For interested readers, images of all sets are included in the supplementary data. Readers are encouraged to view these images while they are being discussed below. All two-dimensional images in the supplementary data were saved using the Cadre X3P Viewer software [11].

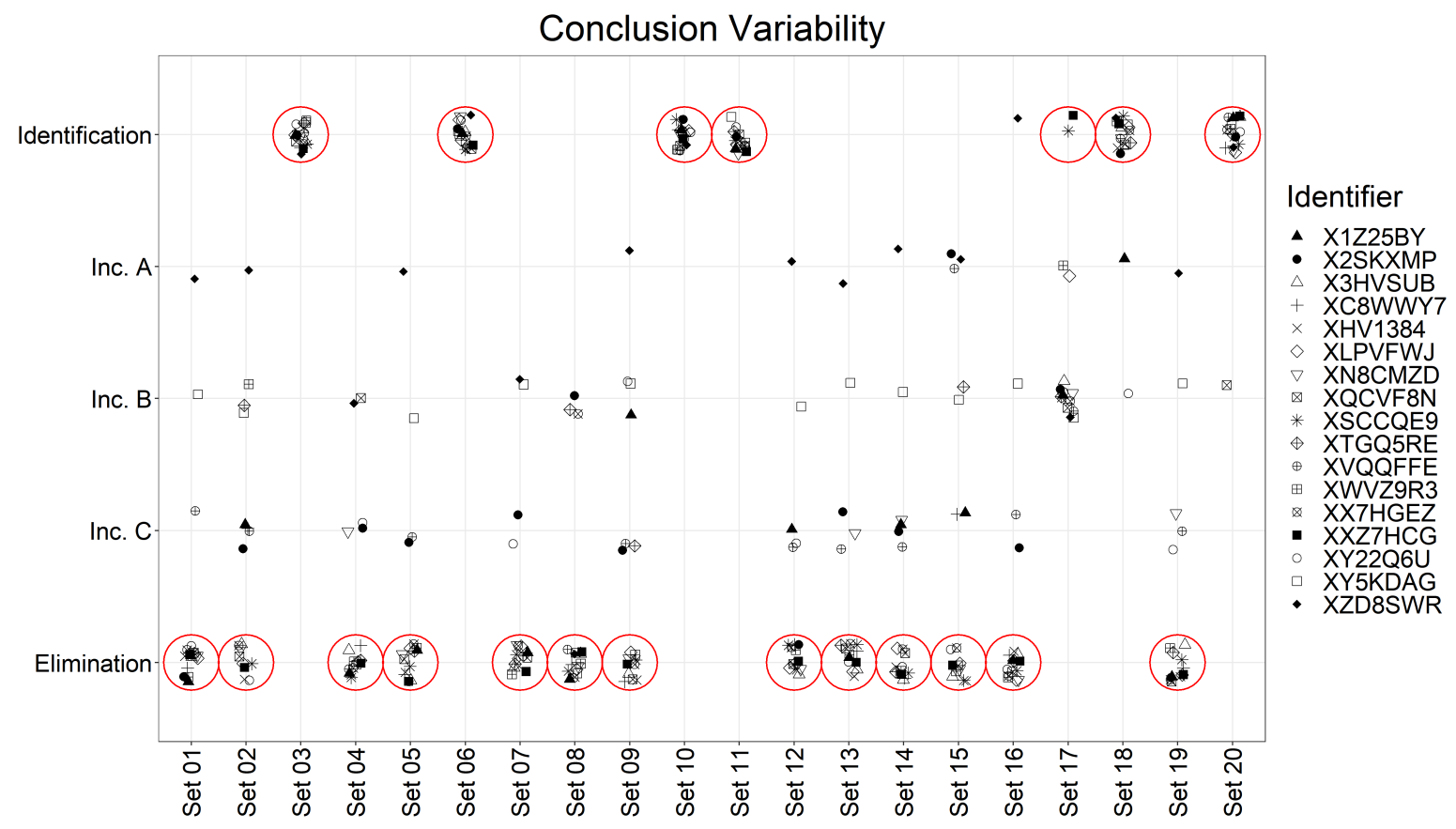

Figure 3: Scatterplot of examiner conclusions where inconclusive conclusions are displayed based on subcategory. The ground truth for each set is circled in red. Data points are jittered to better display the number of points at each conclusion.

Table 3 was created to summarize comparison results for each participant. This table shows the conclusion counts for each of the examiners. Two categories in this table are "Correct Inconclusive" and "Incorrect Inconclusive". These two terms are defined based on the subcategory. For a ground truth identification, an inconclusive A would be a correct inconclusive, while an inconclusive $\mathrm{C}$ would be an incorrect inconclusive. The reverse was true for a ground truth elimination.

Some agencies do not allow examiners to make elimination conclusions when class characteristics are similar. For any set that an inconclusive conclusion was made, a follow-up question asked if agency policy allowed for eliminations on individual characteristics. If the answer to the 
Table 3: Conclusion count variability between participants.

\begin{tabular}{llllllll}
\hline \multirow{2}{*}{ Identifier } & True & True & True & Correct & Incorrect & False & False \\
& Positives & Negatives & Inconclusives & Inconclusives & Inconclusives & Positives & Negatives \\
\hline X1Z25BY & 5 & 8 & 2 & 5 & 0 & 0 & 0 \\
X2SKXMP & 6 & 3 & 2 & 8 & 1 & 0 & 0 \\
XC8WWY7 & 6 & 11 & 1 & 2 & 0 & 0 & 0 \\
XHV1384 & 6 & 13 & 1 & 0 & 0 & 0 & 0 \\
XLPVFWJ & 6 & 13 & 0 & 1 & 0 & 0 & 0 \\
XN8CMZD & 6 & 9 & 1 & 4 & 0 & 0 & 0 \\
XQCVF8N & 5 & 12 & 3 & 0 & 0 & 0 & 0 \\
XSCCQE9 & 7 & 13 & 0 & 0 & 0 & 0 & 0 \\
X3HVSUB & 6 & 13 & 1 & 0 & 0 & 0 & 0 \\
XTGQ5RE & 6 & 9 & 4 & 1 & 0 & 0 & 0 \\
XVQQFFE & 6 & 3 & 1 & 9 & 1 & 0 & 0 \\
XWVZ9R3 & 6 & 12 & 1 & 1 & 0 & 0 & 0 \\
XX7HGEZ & 6 & 12 & 2 & 0 & 0 & 0 & 0 \\
XXZ7HCG & 7 & 13 & 0 & 0 & 0 & 0 & 0 \\
XY22Q6U & 5 & 8 & 3 & 4 & 0 & 0 & 0 \\
XY5KDAG & 6 & 2 & 12 & 0 & 0 & 0 & 0 \\
XZD8SWR & 6 & 1 & 3 & 0 & 9 & 1 & 0 \\
\hline
\end{tabular}

policy question was "No", then the next question asked if they would have made an elimination on this particular set if allowed. Two participants indicated their policy did not allow them to eliminate on individual characteristics. Both of these participants in a total of ten sets adjusted their inconclusive conclusions to eliminations when provided with the opportunity (these eliminations are reflected in Figure 3 and Table 3). In all ten of these instances, the inconclusive subcategory selected was C. Even though the examiners were not able to eliminate based on individual characteristics, they still expressed the degree of dissimilarity that was observed to some extent by using the appropriate subcategory.

These data may justify moving towards a 5-point conclusion scale that replaces the two inconclusive subcategories with their own categories of "support for" either an identification or elimination. In $42.2 \%$ of total inconclusive conclusions, the inconclusive supporting the correct ground truth conclusion was selected. Examiners are observing similarities in support of the ground truth conclusion, but if the similarities are not sufficient to conclude identification or elimination, the current conclusion scale does not have much flexibility to convey the weight of the evidence. The inconclusive subcategories are all neutral in the sense that they are still inconclusives, regardless of whether or not the examiners are attempting to express more value to the result of the comparison. Further research would need to explicitly test a 5-point conclusion scale. It is unknown if the inconclusive $\mathrm{A}$ and $\mathrm{C}$ responses in this study would be inconclusives under a 5-point scale, instead of a "support for" category which may more closely match the wording of the inconclusive subcat- 
egories under the current conclusion scale. Examiners may tend to remain with the inconclusive response depending on the degree of correspondence observed.

To compare examiner conclusions, the results were first analyzed in a similar manner to previous research where inconclusives were not considered incorrect responses. In this way, the only incorrect conclusions were false positives and false negatives. The rationale for this is that an inconclusive is a valid response and is not a false positive or false negative. Using the Cochran test, there was no evidence of significant differences in conclusions across different examiners when inconclusives were considered correct (test statistic $=16.0$, critical value $=26.3$ ). There were no false negatives in this study, but there was one false positive (participant XZD8SWR on Set 16). This may not be an appropriate method of analysis because inconclusive conclusions matter when analyzing variability in responses. The data were next compared based on ground truth accuracy, where the only correct conclusions were ground truth responses. In this way, the inconclusive conclusions were accounted for in the comparison. The Cochran test was significant indicating differences in performance between examiners in ground truth accuracy (test statistic $=103.4$, critical value $=26.3$ ).

To account for the limited sample size of examiners in this study, Figure 4 displays $95 \%$ confidence intervals on the accuracy percentages for each comparison set. The Wilson interval method was used which has improved performance with small sample sizes over the more commonly used Wald interval estimation for the probability of success in binomial distributions [34]. The data in Figure 4 follow correct ground truth responses, meaning inconclusive responses are considered as incorrect for this portion of the analysis. The data point for each set corresponds to the accuracy observed in this study, and the upper and lower confidence bounds are shown. This indicates the range where the "true" accuracy would fall if every firearm examiner could be included in the study. The upper bound of these intervals should ideally be closer to $100 \%$, and a larger sample size of examiners would result in more precise accuracy ranges. The observed accuracy percentages were not because of false positive and false negative conclusions, of which only one false positive was made (Set 16). Common use of inconclusive conclusions was the cause of the observed accuracy values, making up $24.4 \%$ of total conclusions, meaning a lower inconclusive rate would be needed to improve the accuracy of each set. 


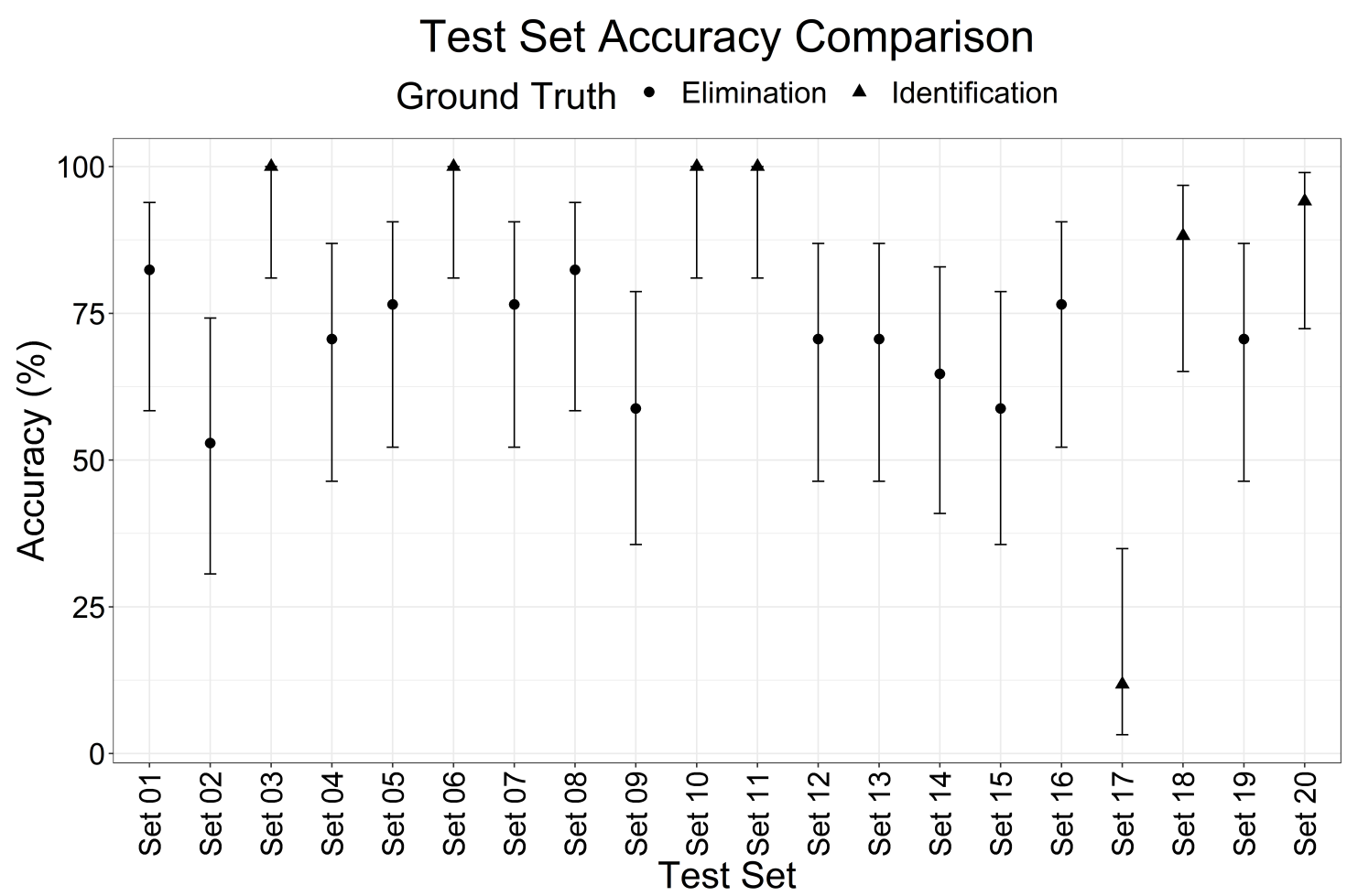

Figure 4: Accuracy percentages in terms of correct ground truth with corresponding $95 \%$ confidence intervals.

\section{Examiner comparisons - consensus analysis}

Recall, there were no statistically significant differences in performance when inconclusives were considered as correct responses, but there were statistically significant differences when only ground truth responses were considered correct. To better account for inconclusive conclusions, SWGFAST guidelines were referenced and non-consensus inconclusives were determined [20]. This allowed for an acceptable range of conclusions for each set based on the IQR of the data. Figure 5 displays this range for each set. Any response which falls within the IQR is considered consensus, and any conclusions which fall outside of the IQR are considered non-consensus, regardless of the ground truth of the set. Table 4 shows the number of consensus and non-consensus conclusions for each participant. The consensus conclusion included the correct ground truth for every set other than Set 17 and for all but one ground truth identification (again, Set 17). Consensus never included the inconclusive subcategory that indicated support for the wrong ground truth conclusion. 


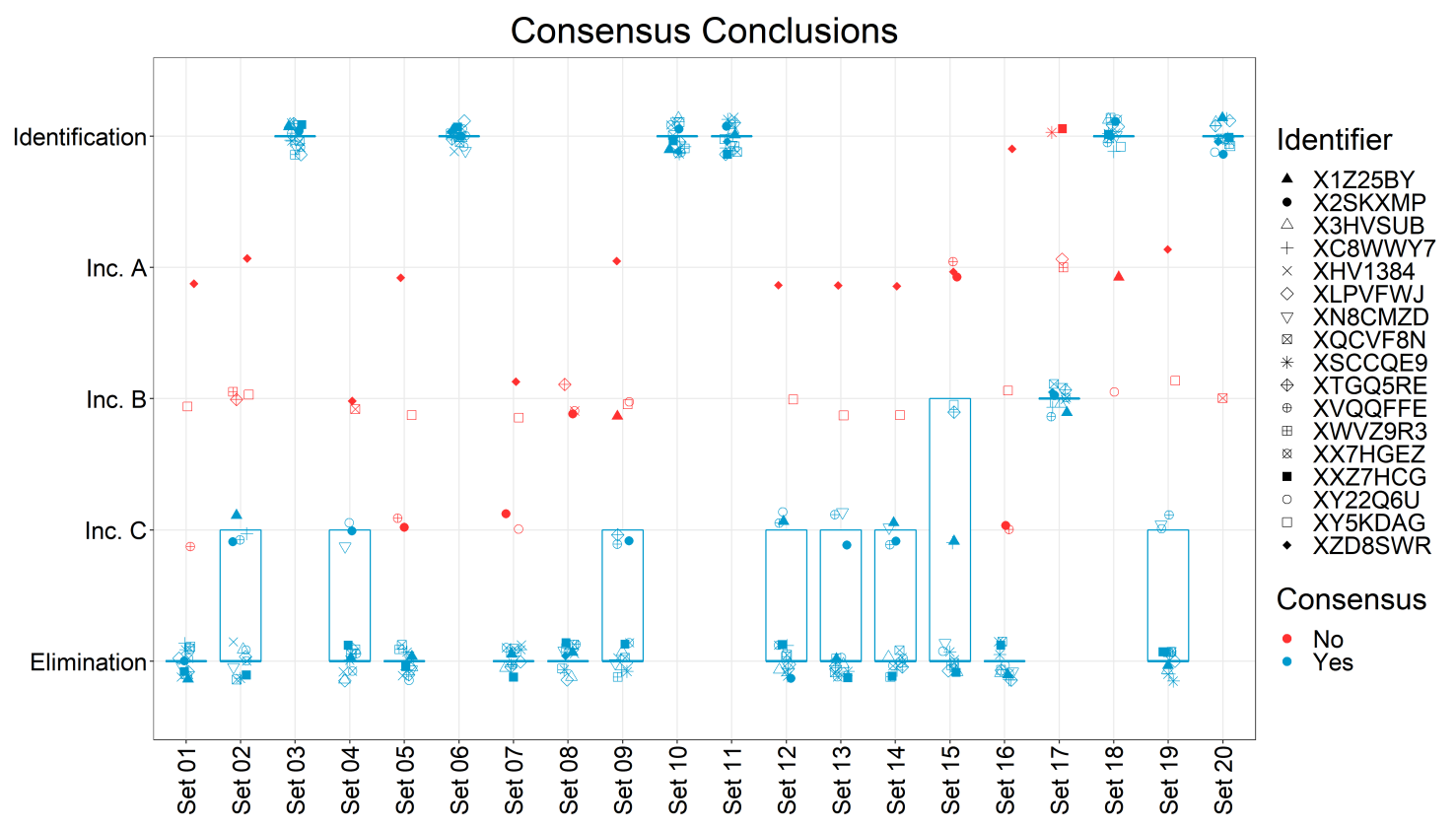

Figure 5: Correct conclusions based on consensus. The boxes show the IQR, blue points are consensus conclusions, and red points are non-consensus conclusions. Data points are jittered to better display the number of points at each conclusion.

Table 4: Consensus conclusion count variability between participants.

\begin{tabular}{lll}
\hline Identifier & Consensus & Non-consensus \\
\hline X1Z25BY & 18 & 2 \\
X2SKXMP & 15 & 5 \\
XC8WWY7 & 20 & 0 \\
XHV1384 & 20 & 0 \\
XLPVFWJ & 19 & 1 \\
XN8CMZD & 20 & 0 \\
XQCVF8N & 18 & 2 \\
XSCCQE9 & 19 & 1 \\
X3HVSUB & 20 & 0 \\
XTGQ5RE & 18 & 2 \\
XVQQFFE & 16 & 4 \\
XWVZ9R3 & 18 & 2 \\
XX7HGEZ & 19 & 1 \\
XXZ7HCG & 19 & 1 \\
XY22Q6U & 17 & 3 \\
XY5KDAG & 10 & 10 \\
XZD8SWR & 8 & 12 \\
\hline
\end{tabular}


Analyzing examiner conclusions based on consensus improved the number of correct ground truth conclusions for 15 of the 17 participants (not counting inconclusives as correct responses). Using participant X1Z25BY as an example, 13 correct ground truth conclusions increased to 18 consensus conclusions. This was because of 7 total inconclusive conclusions where 2 were true inconclusives and 5 were the correct inconclusive. Of those, one true inconclusive and four correct inconclusives were included in the consensus conclusions. Because consensus for Set 17 included only inconclusive B conclusions, it was impossible for any participant to result in $100 \%$ correct ground truth and consensus conclusions. Therefore, two participants had their number of correct conclusions decrease from 20 correct ground truth conclusions to 19 consensus conclusions. However, four participants did end with 20 consensus conclusions. One participant, XZD8SWR, only improved from 7 correct ground truth conclusions to 8 consensus conclusions. This was the only participant to make a false positive, as well as the most incorrect inconclusives with nine. There were only two other incorrect inconclusives across all remaining participants.

In 11 sets (Sets 1, 3, 5, 6, 7, 8, 10,11, 16, 18, and 20), any level of inconclusive was considered a non-consensus response. In Sets 15 (ground truth elimination) and 17 (ground truth identification) a true inconclusive (inconclusive B) was considered an acceptable conclusion. These were the only comparisons where a true inconclusive was included in consensus. There were eight comparison sets, all ground truth eliminations, that included the correct inconclusive (inconclusive $\mathrm{C}$ ) in the consensus conclusion.

The Cochran test was used to compare the performance of the participants across all 20 sets based on consensus. There was evidence of significant differences in performance when all 17 participants were compared (test statistic $=81.3$, critical value $=26.3$ ). Further evidence of differences in participant conclusions can be seen by considering the number of non-consensus responses per set. For example, a maximum of $23.5 \%$ of responses were non-consensus for six sets $(2,5,7,9$, 16 , and 17).

Inconclusive can be a cautious response because the examiner will never result in a false positive or false negative conclusion. However, as stated previously, there are potential consequences with inconclusives. As the number of inconclusives increases, so does the potential number of missed identifications and eliminations. If there is enough information for the correct ground truth conclusion to be made then the examiner must make a decision about the source of the unknown cartridge case(s) [35]. Consensus is more difficult to assess in casework when a single examiner (potentially multiple with a verification step) analyzes the evidence. However, training using a similar approach to that of this study, where there is consensus on each set as to when inconclusives are and are not appropriate, may be moving in the correct direction. 


\section{Examiner comparisons - ten cartridge case set}

The results for the ten cartridge case set are displayed in Figure 6, where the two-letter strings refer to cartridge case identifiers. The correct number of firearms is five, and the correct groupings can be seen back in Table 2. Recall, participants were asked to determine the number of firearms that fired the ten cartridge cases. The maximum number was ten if each cartridge case was placed in a group by itself. In Figure 6, the y-axis shows the firearm number. This number is arbitrary. As long as the correct cartridge cases are grouped together, the firearm number is irrelevant. Therefore, the grouping for Firearm 1 may be different between participants, and every participant could get the grouping correct. Each cartridge case identifier circled in red indicates a cartridge case that was not placed in the correct group.

\section{Cartridge Case Set Conclusions}

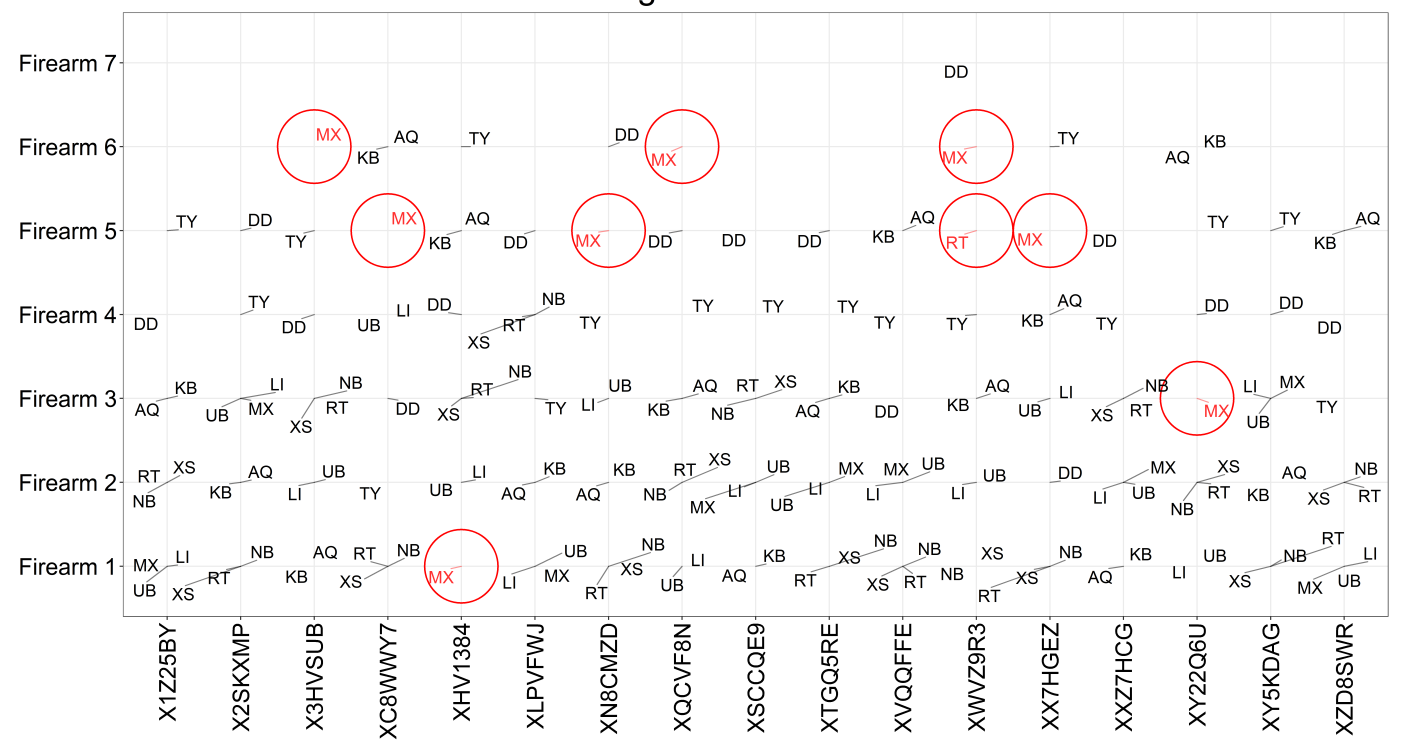

Figure 6: Cartridge case groupings for Set 21.

All of the cartridge cases were grouped with the correct five firearms by $52.9 \%$ of participants. However, the cartridge cases were grouped into six firearms by $41.2 \%$ of participants and seven firearms by the one remaining participant. These results indicate there is variability in how examiners perform on comparisons where no known firearm is present. The seven participants which grouped the cartridge cases into six firearms all incorrectly placed cartridge case MX into a group by itself, therefore making the same error indicating difficulty with that particular cartridge case. The participant which grouped the cartridge cases into seven firearms made the same error as previously stated with cartridge case MX but also incorrectly grouped cartridge case RT by itself. It is important to note that a Ruger LC9 fired cartridge case MX, the same model as Set 17 (but a 
different serial number) which was the most difficult set of the study. These model firearms seem to present a challenge to examiners.

Some clarification is needed here. Every "error" made in this set was simply placing a single cartridge case into its own grouping rather than with the other cartridge cases with which it should have been grouped, and it is important to note that no cartridge cases were grouped with cartridge cases fired from a separate firearm. Once suspect firearms are recovered and submitted to the laboratory, comparisons such as those from Sets 1 - 20 would be performed. At that time it may become apparent that a cartridge case grouped by itself actually was fired by the same firearm which fired the other cartridge cases belonging to that group. Therefore, the cartridge cases grouped by themselves were not considered as errors, meaning no errors were made in this set.

These cartridge cases placed into their own groups may be cautious responses by the participants. A confidence rating for each group would have been extremely useful for this set in particular to determine if these are analogous to the inconclusive conclusions made in some of the previous 20 test sets. For example, two cartridge cases, DD and TY, were the only cartridge cases composing the set fired from their respective firearms. A high confidence rating for these two cartridge cases would indicate the examiners were confident that these cartridge cases belonged in their own groups. Alternatively, a low confidence rating for cartridge case MX from the examiners which grouped it into its own firearm would indicate a higher probability it may have been fired by the same firearm as other cartridge cases from this set. Confidence ratings are a necessity in future research of similar design.

\section{Examiner cost}

To investigate the cost of different examiners in their conclusions, a value was assigned to each conclusion on a scale of 0 to 100. True positives and true negatives were both assigned a cost of 0 because those are correct ground truth conclusions. A false positive was assigned the highest value of 100 , while a false negative was slightly less at 75 . The inconclusives were in-between these extremes. A correct inconclusive was given a value of 15, an incorrect inconclusive was given a value of 50, and a true inconclusive was near the middle at 30. The magnitude of the selected values are simply being used as a relative scale. For each participant the number of conclusions in a given category that were made were multiplied by the cost and then summed. For example, an examiner that made all seven true positives and 11 of the 13 true negatives with two correct inconclusives would have a total cost of 30 . Values closer to 0 indicate less cost and therefore better overall performance.

Figure 7 displays the cost of each examiner based on years of experience and the approximate number of cases worked per year. The expected trend may be observing higher cost in examiners with less experience and a lower cost from those with more experience. This was not the case here. 
The largest cost was from an examiner with more than ten years of experience due to one false positive and nine non-consensus inconclusive conclusions.

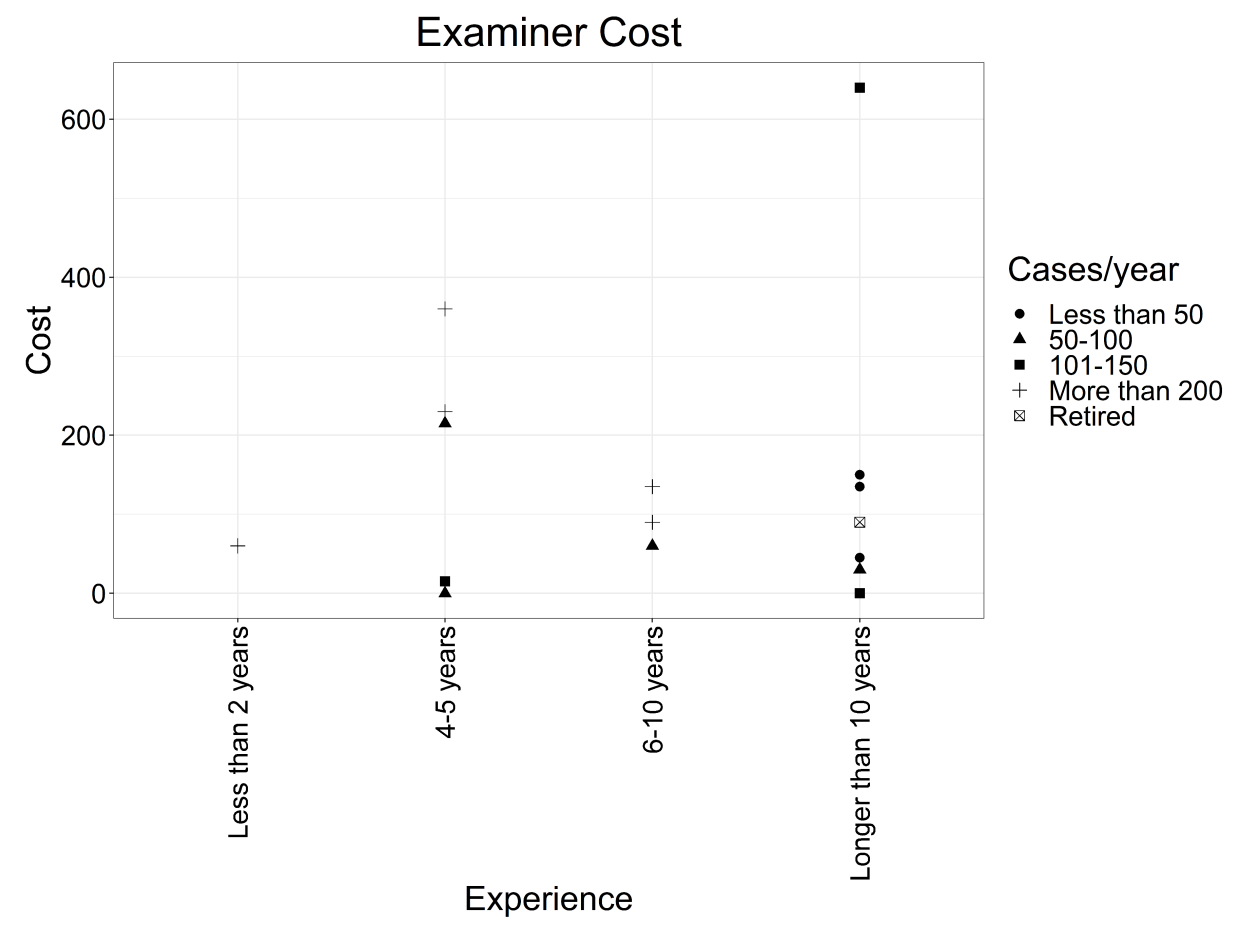

Figure 7: Total cost of conclusions by each examiner over all 20 test sets.

\section{Double-cast quality}

For each comparison set, participants were able to report and describe any quality issues with the double-casts. There were five reported issues resulting in $0.37 \%$ of total double-casts distributed that had a quality issue. However, no participants requested a replacement. Four of these five cases still resulted in the correct ground truth conclusion, indicating those quality issues did not interfere significantly with the comparison. In the fifth case the examiner concluded an inconclusive $\mathrm{C}$ on a ground truth elimination. The reported issue for this inconclusive was that the smooth appearance of the firing pin impression of the questioned cartridge case in Set 1 may be due to the casting process. Based on the master cartridge cases, the firing pin of the questioned cartridge case is relatively smooth compared to the three knowns. However, this may still have contributed to the inconclusive $\mathrm{C}$ conclusion instead of the examiner making an elimination.

One of the double-cast test packets was examined by two participants. Participant XY22Q6U was the first to examine their set. Once returned and checked, the set was then sent to participant XXZ7HCG. All casts were in the proper bags and clean when returned the first time, meaning no 
adjustments were made to the set prior to being sent to the subsequent participant. For this set sent to XY22Q6U and XXZ7HCG, there were 13 and 20 correct ground truth conclusions, respectively. These both resulted in 19 correct when comparing to consensus conclusions. If there was set-toset variability within the casts that was to affect examiner conclusions, it would be expected that examiners that analyzed the same sets would perform equally based on both ground truth and consensus. That was not the case here, and therefore differences in performance may be due to the examiners and not cast variability.

\section{Automated comparison results}

Automated comparison results for each set are displayed in Figure 8. It is known that the CMC metric may result in differing numbers of congruent cells depending on whether a comparison is run from Surface A to Surface B or from Surface B to Surface A [36]. For the test set cartridge case results here, the questioned cartridge cases were compared to the known cartridge cases because, fundamentally, the evidence should be compared to the knowns and not the other way around [37]. In Figure 8, the "+" marks represent the known to known comparisons and the " $\times$ " marks represent the questioned to known comparisons.

Performance of each sample firearm is also included in Figure 8. Ten cartridge cases were fired from each firearm and inter-compared for a total of 45 known match comparisons. These ten cartridge cases were then compared to 10 cartridge cases from each of the remaining 25 firearms used throughout this study for a total of 2500 known non-match comparisons. These data are shown in Figure 8 as blue circles and red triangles to represent known match and known non-match comparisons, respectively. Although the examiner participants in the study were not provided the performance of each sample firearm, the data were included here as a reference for each firearm. The percentages shown on each panel of Figure 8 will be discussed in the Automated Comparison Interpretation section below.

Table 5 shows the ROC curve AUC values that were less than one in either metric for the sample firearm of each set. The AUC is shown for CMC and ACCF results to illustrate performance utilizing both metrics. Performance was generally high for all firearms regardless of the metric chosen. Thirteen of 20 AUC values were equal to one for the congruent cell results, and 14 of 20 were equal to one for the ACCF results. The overall worst performing firearm was the Ruger LC9 used in Set 17 with CMC and ACCF AUC values of 0.5346 and 0.7572, respectively. All remaining AUCs were between 0.9000 and 1.000. These results indicate high discriminating power when comparing 3D surfaces using the CMC method. 


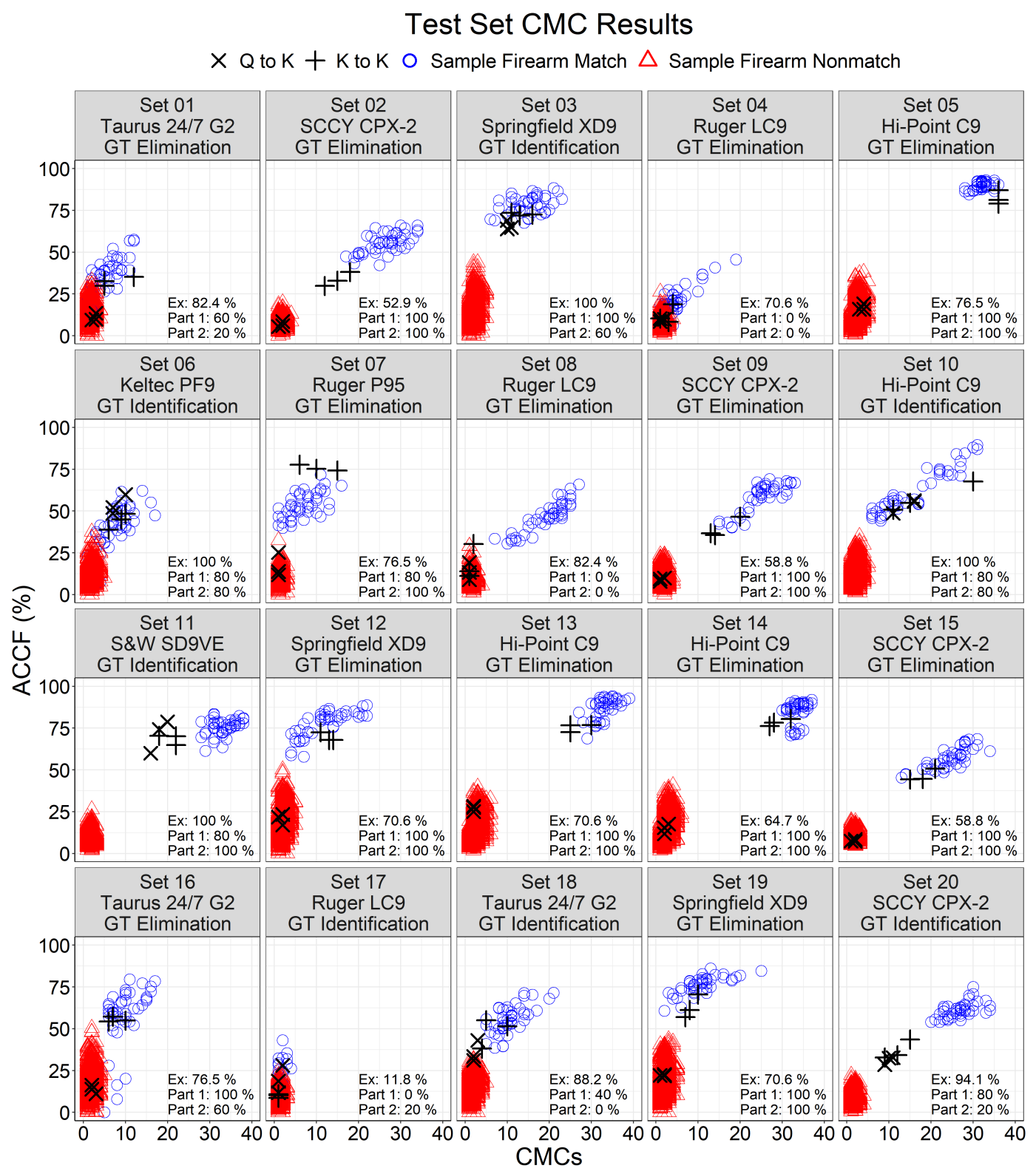

Figure 8: Automated comparison results for the 20 examiner test sets.

The CMC and ACCF results display their potential as a case-specific system to assist examiners. For example, Set 3, a Springfield XD9 identification comparison, shows the K to K and Q to $\mathrm{K}$ comparisons grouped closely together indicating the questioned cartridge case was fired by the same firearm as the known cartridge cases. Similarly for an elimination comparison, Set 5, a Hi-Point C9, shows complete separation between the $\mathrm{K}$ to $\mathrm{K}$ and $\mathrm{Q}$ to $\mathrm{K}$ comparisons. There are some sets where it is more difficult to ascertain the ground truth from the algorithm results. Set 17 is the best example of a difficult comparison as indicated by the fact that the $\mathrm{K}$ to $\mathrm{K}$ and $\mathrm{Q}$ to 
Table 5: Test set AUC values for automated comparison results.

\begin{tabular}{lll}
\hline Set Number & CMC AUC & ACCF AUC \\
\hline 1 & 0.9043 & 0.9419 \\
4 & 0.9403 & 0.9104 \\
6 & 0.9781 & 0.9813 \\
7 & 0.9201 & 0.9900 \\
12 & 0.9991 & 1.0000 \\
16 & 1.0000 & 0.9432 \\
17 & 0.5346 & 0.7572 \\
19 & 0.9890 & 1.0000 \\
\hline
\end{tabular}

$\mathrm{K}$ comparisons are all overlapping and clustered near the origin of the $\mathrm{CMC}$ and $\mathrm{ACCF}$ axes. The cartridge cases from this particular Ruger LC9 have smooth breech face impressions. Based on the sample firearm performance, there are no reproducible individualizing features for the algorithm to be able to separate these cartridge cases from cartridge cases fired by other firearms.

\section{Automated comparison interpretation}

Recall, the automated comparison data were shown to five students to assess interpretation of the results. Students were selected due to familiarity with the NIST algorithms and because these algorithms are for research purposes and therefore not currently in use by firearm examiners. The purpose was to evaluate how indicative the algorithm results were of the ground truth of the set. The automated comparison interpretation results for Part 1 are displayed in Figure 9 and summarized in Table 6. One participant exhibited worse performance than the others. This participant, X8CNG6A, only made one of seven true positives, eight true inconclusives, and one false positive. There were no other false positives or negatives for Part 1, and the remaining four participants made between five and six true positives with less inconclusives. However, two participants, XA8BBH4 and XYAYLM9, made two incorrect inconclusives each.

The automated comparison interpretation results for Part 2 are displayed in Figure 10 and summarized in Table 7. Two additional false positives were made in Part 2, both from participant XYAYLM9. Furthermore, there were three false negatives. Two were made by participant X8CNG6A and one was made by participant XUCFULF. It was expected that additional data on the sample firearm performance would improve the results, but this was not the case. The number of true positives increased from one to two for participant X8CNG6A, however, the number of true positives decreased for each of the remaining four participants. The number of true negatives either stayed the same (participants XA8BBH4 and XYAYLM9) or decreased compared with Part 1. 
Going back to Figure 8, the percent of correct ground truth conclusions for Part 1 and Part 2 are displayed for each set. When background firearm information was included, five sets $(1,3,16,18$, and 20) decreased in correct ground truth percentage, three sets $(7,11$, and 17) increased in correct ground truth percentage, and 12 sets (all remaining) did not change. Eight sets had 100\% correct for both Part 1 and Part 2, indicating the overall opinion of the participants did not change when background information was available. Additionally, four sets had 100\% for either Part 1 or Part 2 , but the accuracy either increased or decreased with the addition of sample firearm performance. Two sets had 0\% accuracy for both Part 1 and Part 2, and both of these sample firearms were Ruger LC9s. Two additional sets had $0 \%$ accuracy for either Part 1 or Part 2, and increased from 0\% or decreased to $0 \%$ with the additional firearm information.

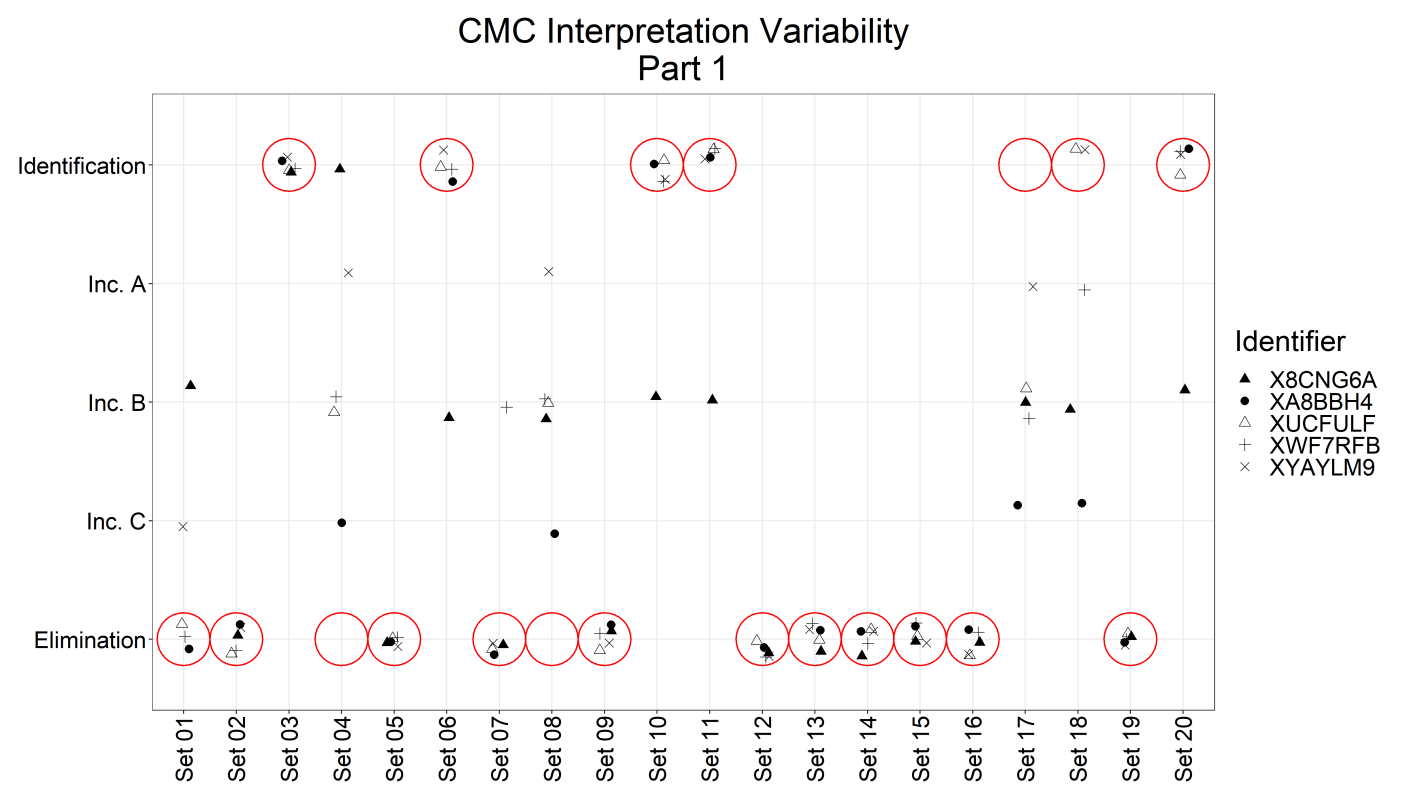

Figure 9: Scatterplot of automated comparison interpretation Part 1 conclusions. Ground truth conclusions are circled in red. Data points are jittered to better display the number of points at each conclusion.

Table 6: Automated comparison interpretation Part 1 participant results.

\begin{tabular}{llllllll}
\hline Identifier & $\begin{array}{l}\text { True } \\
\text { Positives }\end{array}$ & $\begin{array}{l}\text { True } \\
\text { Negatives }\end{array}$ & $\begin{array}{l}\text { True } \\
\text { Inconclusives }\end{array}$ & $\begin{array}{l}\text { Correct } \\
\text { Inconclusives }\end{array}$ & $\begin{array}{l}\text { Incorrect } \\
\text { Inconclusives }\end{array}$ & $\begin{array}{l}\text { False } \\
\text { Positives }\end{array}$ & $\begin{array}{l}\text { False } \\
\text { Negatives }\end{array}$ \\
\hline X8CNG6A & 1 & 10 & 8 & 0 & 0 & 1 & 0 \\
XA8BBH4 & 5 & 11 & 0 & 2 & 2 & 0 & 0 \\
XUCFULF & 6 & 11 & 3 & 0 & 0 & 0 & 0 \\
XWF7RFB & 5 & 10 & 4 & 1 & 0 & 0 & 0 \\
XYAYLM9 & 6 & 10 & 0 & 2 & 2 & 0 & 0 \\
\hline
\end{tabular}




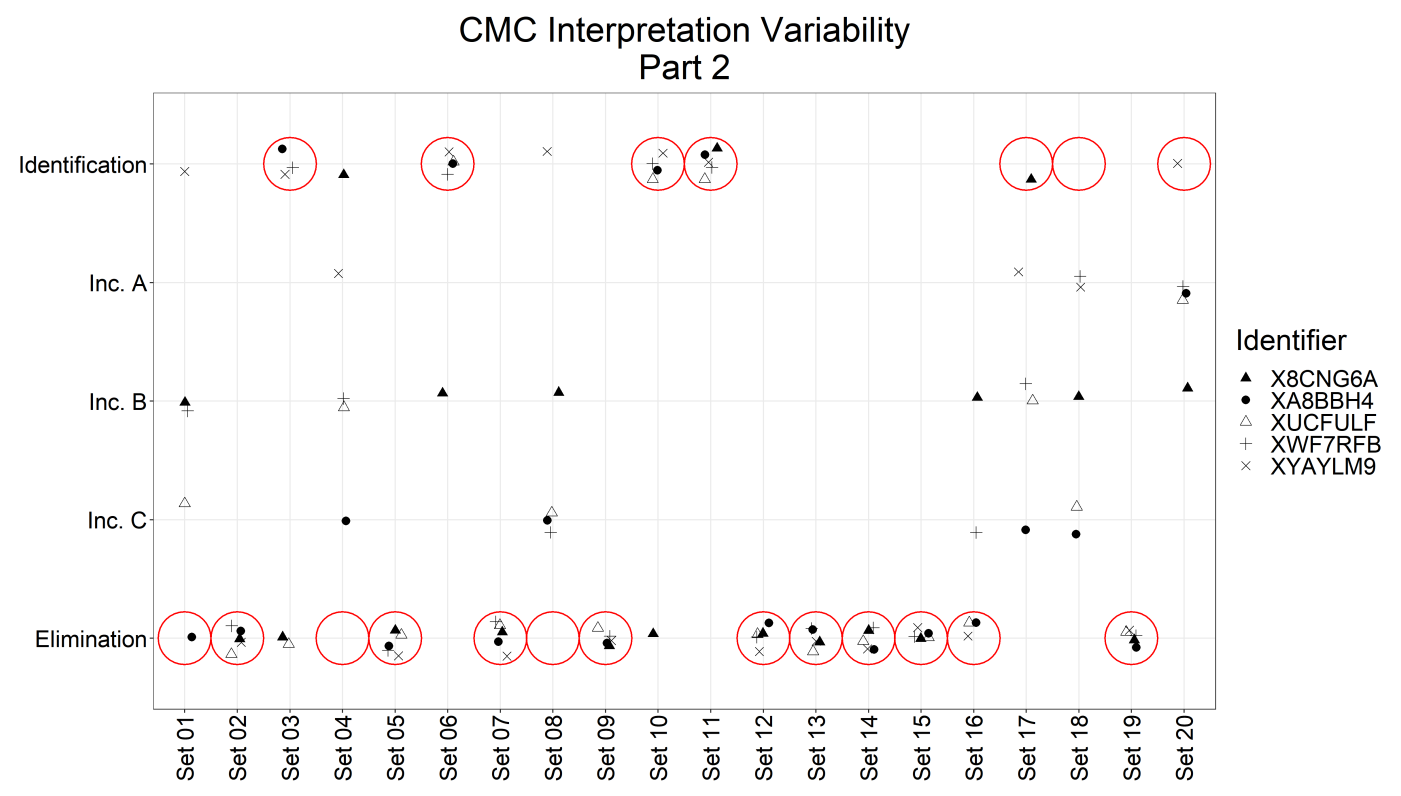

Figure 10: Scatterplot of automated comparison interpretation Part 2 conclusions. Ground truth conclusions are circled in red. Data points are jittered to better display the number of points at each conclusion.

Table 7: Automated comparison interpretation Part 2 participant results.

\begin{tabular}{llllllll}
\hline Identifier & $\begin{array}{l}\text { True } \\
\text { Positives }\end{array}$ & $\begin{array}{l}\text { True } \\
\text { Negatives }\end{array}$ & $\begin{array}{l}\text { True } \\
\text { Inconclusives }\end{array}$ & $\begin{array}{l}\text { Correct } \\
\text { Inconclusives }\end{array}$ & $\begin{array}{l}\text { Incorrect } \\
\text { Inconclusives }\end{array}$ & $\begin{array}{l}\text { False } \\
\text { Positives }\end{array}$ & False \\
& Negatives \\
\hline X8CNG6A & 2 & 9 & 6 & 0 & 0 & 1 & 2 \\
XA8BBH4 & 4 & 11 & 0 & 3 & 2 & 0 & 0 \\
XUCFULF & 3 & 10 & 2 & 3 & 1 & 0 & 1 \\
XWF7RFB & 4 & 9 & 3 & 4 & 0 & 0 & 0 \\
XYAYLM9 & 5 & 10 & 0 & 2 & 1 & 2 & 0 \\
\hline
\end{tabular}

Overall, the addition of sample firearm performance was not helpful. There was only one false positive conclusion in Part 1 (Set 4), but in Part 2 there were two additional false positives (Sets 1 and 8) and three false negatives (Sets 3 and 10). These results were unexpected, as the addition of sample firearm information was hypothesized to assist in the interpretation of the automated comparison results. It was surprising to see participants become less confident in the ground truth compared to Part 1, which was especially apparent in Set 3. The percentage correct decreased from $100 \%$ to $60 \%$ with the addition of sample firearm information. However, this additional information displays complete separation between the matches and non-matches.

To compare examiner conclusions to the automated comparison interpretation results, Figure 8 also displays the percent correct ground truth results from the examiner comparisons. These are the accuracy percentages observed from the examiners in this study (refer back to Figure 4 for 
the corresponding $95 \%$ confidence intervals). There were no sets with $100 \%$ correct ground truth responses for examiners, Part 1, and Part 2. However, there was one set, Set 3, where all examiners and Part 1 interpretation came to the correct ground truth conclusion. Part 1 is a more fair comparison to the examiner conclusions because examiners would not typically have data on the performance of a suspect firearm outside of the few test fires performed, and in this study they did not have access to this information. For nine sets, examiners performed better than interpretation of Part 1 data. In ten sets, Part 1 interpretation led to more correct ground truth conclusions. The purpose of the automated comparisons here is not to perform a direct comparison to the results of the examiners. The goal is to show the benefit of using a system such as the NIST algorithms in correcting errors in some sets. These results indicate that both methods can be utilized in tandem to produce stronger overall conclusions than either in isolation.

When comparing the examiner results to the automated comparison results it is important to consider that only the breech face area is being utilized in the automated comparisons here. This is particularly noticeable when examining the results from Sets 4 and 8, both ground truth eliminations. The sample firearm for both of these sets is a Ruger LC9, the same model as Set 17 where a majority of participants made an inconclusive conclusion. However, the firing pin impressions between the questioned and known cartridge cases are different in appearance between the Q and Ks for both Sets 4 and 8 . Therefore, examiners performed well on these two sets while the algorithms did not. CMC results for the breech face and firing pin impression areas have been successfully combined and is an area of further study to continue to improve the discriminating power of the algorithm [38].

In casework, automated comparison systems, such as the ACCF and CMC metrics, could be useful as a quality control measure to help reduce errors. Previous research in fingerprints used a case-specific AFIS system in a similar manner [35]. For example, the cartridge cases from Set 16 show up in a case. In the examiner portion of this study, a false positive resulted. If the participant that made the identification conclusion were to write up the report, this would be a false positive error. The error would potentially be corrected during a verification procedure. However, regardless of the verification policy, if the cartridge cases were compared using a case-specific system such as the NIST algorithms, the automated comparison results would indicate that an identification was the incorrect conclusion (refer to Figure 8). The examiner could then go back and examine the cases further. This would all need to be documented, as there is the potential for automated comparison results to bias examiners. The automated comparisons could even be performed by the verifier instead of the examiner originally assigned to the case to further reduce any effects of bias. 


\section{Conclusions}

When considering inconclusive conclusions as correct conclusions, there were no statistically significant differences in performance between examiners due to only one error being made. However, when considering only ground truth conclusions as correct there was evidence of significant differences in examiner performance. To account for the inconclusive subcategories, consensus conclusions were determined for each set. There were significant differences in performance when comparing examiners based on consensus. Furthermore, analyzing consensus conclusions resulted in up to $23.5 \%$ of conclusions being non-consensus for a given set.

Due to the adversarial nature of the legal system and the potential this research will be utilized in such a setting, it is important to clarify a few key points. The conclusion variabilty that was observed does not support broader claims about poor performance by all firearms examiners in all previously performed comparisons. Some variability in conclusions was expected, and it is also notable that this variation was almost exclusively contained within the inconclusive categories and not more widely observed to include many false positive and false negative conclusions. Furthermore, the sole false positive conclusion in the study indicates that particular comparison was not a wide-spread issue amongst examiners.

The observed ground truth accuracy from the comparison sets in this study would ideally be higher, meaning less overall inconclusive conclusions. Furthermore, the confidence intervals resulted in a wide range of ground truth accuracy for each set, where the correct ground truth conclusions were the only responses considered as correct, due to the small sample size of examiners. Obviously, a higher observed ground truth accuracy is desired and a larger sample size of examiners would further refine the confidence intervals to produce more precise estimates of the "true" accuracy. Because there was only one false positive in the study, a lower overall inconclusive rate would be needed to achieve a higher observed accuracy. In this study the total inconclusive rate (considering A, B, and C subcategories) was $24.4 \%$. When only considering inconclusive B conclusions this rate decreases to $10.9 \%$. Therefore, a 5-point conclusion scale may lead to less total inconclusive conclusions, although this needs to explicitly be tested.

Automated comparison metrics were shown to have a high discriminating power with the test sets presented to examiners. These results were generally indicative of the ground truth in each set based on interpretation by students, although some errors were made. As an area for further study, automated comparison results should be interpreted by experienced examiners. Incorporating a case-specific automated comparison algorithm as a quality control measure may assist in correcting any errors, and combined with examiner conclusions, would strengthen the overall interpretation of the evidence. 
The successful application of double-casts in this study should promote continued use for creating training and testing sets. For training sets, double-casts should be made from comparisons identified through research to be problematic. This could include Set 16 from this study because a false positive was made, as well as Set 17 for when an inconclusive may be the most appropriate response. Proficiency tests could be conducted in a similar fashion. That would ensure examiner proficiency is being tested based on the same comparisons for all test takers, and the level of difficulty between examiners is controlled. In this way, double-casting could lead to standardization with respect to training and testing which does not currently exist.

\section{Acknowledgments}

The authors would like to sincerely thank all examiners and students that volunteered to participate in this study. The authors would like to acknowledge use of the supercomputing system Thorny Flat at WVU, which is funded in part by the National Science Foundation EPSCoR Research Infrastructure Improvement Cooperative Agreement \#1003907, National Science Foundation Major Research Instrumentation Program (MRI) Award \#1726534, the state of West Virginia (WVEPSCoR via the Higher Education Policy Commission) and WVU. The authors would also like to acknowledge the firearm and tool mark analysis team at the National Institute of Standards and Technology (NIST) for providing access to their tool mark research software. The software was applied in this paper to calculate the ACCF and CMC comparison results.

\section{References}

[1] National Academy of Sciences. Strengthening Forensic Science in the United States: A Path Forward. The National Academies Press, 2009.

[2] President's Council of Advisors on Science and Technology. Forensic Science in Criminal Courts: Ensuring Scientific Validity of Feature-Comparison Methods, 2016.

[3] Stephen G. Bunch and Douglas P. Murphy. A comprehensive validity study for the forensic examination of cartridge cases. AFTE Journal, 35(2):201-203, 2003.

[4] Dennis J. Lyons. The identification of consecutively manufactured extractors. AFTE Journal, 41(3):246-256, 2009.

[5] Dawn LaPorte. An empirical and validation study of breechface marks on .380 ACP caliber cartridge cases fired from ten consecutively finished Hi-Point C9 pistols. AFTE Journal, 43(4):303-309, 2011.

[6] Thomas G. Fadul Jr, Gabriel A. Hernandez, Stephanie Stoiloff, and Sneh Gulati. An empirical study to improve the scientific foundation of forensic firearm and tool mark 
identification utilizing 10 consecutively manufactured slides. AFTE Journal, 45(4):376-393, 2013.

[7] Erich D. Smith. Cartridge case and bullet comparison validation study with firearms submitted in casework. AFTE Journal, 36(4):130-135, 2004.

[8] David P. Baldwin, Stanley J. Bajic, Max Morris, and Daniel Zamzow. A study of false-positive and false-negative error rates in cartridge case comparisons. Retrieved from https://www.ncjrs.gov/pdffiles1/nij/249874.pdf, 2014.

[9] Angela Stroman. Empirically determined frequency of error in cartridge case examinations using a declared double-blind format. AFTE Journal, 46(2):157-175, 2014.

[10] Tasha P. Smith, G. Andrew Smith, and Jeffrey B. Snipes. A validation study of bullet and cartridge case comparisons using samples representative of actual casework. Journal of Forensic Sciences, 61(4):939-946, 2016.

[11] Cadre Forensics. X3P Viewer Software. Available by request at www.cadreforensics.com as of 21-Feb-2020.

[12] Petra Pauw-Vugts, Alice Walters, Leif Øren, Kripos, and Leopold Pfoser. Faid2009: Proficiency test and workshop. AFTE Journal, 45(2):115-127, 2013.

[13] Pierre Duez, Todd Weller, Marcus Brubaker, Richard E. Hockensmith, and Ryan Lilien. Development and validation of a virtual examination tool for firearm forensics. Journal of Forensic Sciences, 63(4):1069-1084, 2018.

[14] Erwin J.A.T. Mattijssen, Cilia L.M. Witteman, Charles E.H. Berger, Nicolaas W. Brand, and Reinoud D. Stoel. Validity and reliability of forensic firearm examiners. Forensic Science International, 307, 2020.

[15] Eric Law and Keith Morris. The utility of double-casting for creating cartridge case reproductions. AFTE Journal, 52(1):26-39, 2020.

[16] Eric F. Law and Keith B. Morris. Three-dimensional analysis of cartridge case double-casts. Journal of Forensic Sciences, September 2020.

[17] Keith B. Morris, Eric F. Law, Roger L. Jefferys, and Elizabeth C. Dearth. Interpretation of cartridge case evidence using IBIS and Bayesian networks. Technical report, Department of Forensic and Investigative Science, West Virginia Univeristy, 2016.

[18] Qualtrics. https://www.qualtrics.com. Last accessed 21-Feb-2020.

[19] AFTE. AFTE criteria for identification committee final report. AFTE Journal, 24(3), 1992.

[20] Scientific Working Group on Friction Ridge Analysis, Study, and Technology (SWGFAST). Document \#15. Standard for the Definition and Measurement of Rates of Errors and Non-Consensus Decisions in Friction Ridge Examination. Ver 2.0, 2012. 
[21] David J. Brundage. The identification of consecutively rifled gun barrels. AFTE Journal, 30(3):438-444, 1998.

[22] Charles S. DeFrance and Michael D. Van Arsdale. Validation study of electrochemical rifling. AFTE Journal, 35(1):35-37, 2003.

[23] Joseph L. Peterson and Penelope N. Markham. Crime laboratory proficiency testing results, 1978-1991, II: Resolving questions of common origin. Journal of Forensic Sciences, 40(6):1009-1029, 1995.

[24] James E. Hamby, David J. Brundage, Nicholas D. K. Petraco, and James W. Thorpe. A worldwide study of bullets fired from 10 consecutively rifled 9 MM RUGER pistol barrels - analysis of examiner error rate. Journal of Forensic Sciences, 64(2):551-557, 2018.

[25] Itiel E. Dror and Glenn Langenburg. "Cannot decide": The fine line between appropriate inconclusive determinations versus unjustifiably deciding not to decide. Journal of Forensic Sciences, 64(1):10-15, 2018.

[26] W. J. Conover. Practical Nonparametric Statistics. Wiley Series in Probability and Mathematical Statistics. John Wiley and Sons, 2nd edition, 1980.

[27] T. V. Vorburger, J. Song, and N. Petraco. Topography measurements and applications in ballistics and tool mark identifications. Surface Topography: Metrology and Properties, 4(1), 2015.

[28] Open Forensic Metrology Consortium. OpenFMC \& X3P. Available at https://www.cadreforensics.com/x3p.html as of August 21, 2019.

[29] John Song. Proposed nist ballistics identification system (nbis) based on 3D topography measurements on correlation cells. AFTE Journal, 45(2):184-194, 2013.

[30] W. Chu, M. Tong, and J. Song. Validation tests for the congruent matching cells (CMC) method using cartridge cases fired with consecutively manufactured pistol slides. AFTE Journal, 45(4):361-366, 2013.

[31] John Song, Theodore V. Vorburger, Wei Chu, James Yen, Johannes A. Soons, Daniel B. Ott, and Nien Fan Zhang. Estimating error rates for firearm evidence identifications in forensic science. Forensic Science International, 284:15-32, 2018.

[32] Tom Fawcett. ROC graphs: Notes and practical considerations for data mining researchers. Intelligent Enterprise Technologies Laboratory. HP Laboratories Palo Alto, 2003.

[33] U.S. Department of Health and Human Services, Food and Drug Administration, Office of the Commissioner (OC), Good Clinical Practice Program (GCPP). Guidance for Sponsors, Clinical Investigators, and IRBs. Data Retention When Subjects Withdraw from FDA-Regulated Clinical Trials, 2008.

[34] Lawrence D. Brown, T. Tony Cai, and Anirban DasGupta. Confidence intervals for a binomial proportion and asymptotic expansions. The Annals of Statistics, 30(1):160-201, 2002. 
[35] Glenn Langenburg, Carey Hall, and Quincy Rosemarie. Utilizing AFIS searching tools to reduce errors in fingerprint casework. Forensic Science International, 257:123-133, 2015.

[36] Zhe Chen, John Song, Wei Chu, Johannes A. Soons, and Xuezeng Zhao. A convergence algorithm for correlation of breech face images based on the congruent matching cells (CMC) method. Forensic Science International, 280:213-223, 2017.

[37] Itiel E. Dror, William C. Thompson, Christian A. Meissner, Irv Kornfield, Dan Krane, Michael Saks, and Michael Risinger. Letter to the editor- context management toolbox: A linear sequential unmasking (LSU) approach for minimizing cognitive bias in forensic decision making. Journal of Forensic Sciences, 60(4):1111-1112, 2015.

[38] Daniel Ott, Robert Thompson, and Junfeng Song. Applying 3D measurements and computer matching algorithms to two firearm examination proficiency tests. Forensic Science International, 271:98-106, 2016. 


\title{
5. Bayesian network for the analysis of firearm examiner conclusions
}

\section{Publication status}

This chapter is in final preparations for submission to a forensic science journal.

\begin{abstract}
Assessing error rates in cartridge case comparisons is important because firearm examiners use subjective methods to make conclusions. Error rate studies typically consist of test fired cartridge cases sent to participating examiners. However, due to shot-to-shot variability examiners may receive test sets with various difficulty levels. Therefore, results between examiners are unable to be directly compared. For this reason, double-casts were used in this study to ensure participants were receiving test sets with the same level of detail. Twenty master test sets were created from various firearms that consisted of three questioned cartridge cases and one unknown cartridge case. Double-casts were then created of all master test sets. The double-casts ensured that participants received test sets with the same level of detail. Two Bayesian network models were created from the examiner conclusion data. One network was created manually based on knowledge of the variable relationships. A second model was created using the tree augmented naive Bayes (TAN) algorithm. These models were evaluated using leave-one-out cross validation and receiver operating characteristic (ROC) curves. Based on those results, the manually created network was selected for continued analysis. Using the selected Bayesian network, likelihood ratios (LRs) were calculated based on the examiner conclusions. These results showed that their conclusions were generally in support of the ground truth conclusion. The magnitude of effects of additional variables on the conclusion LRs was then analyzed. These data showed that additional information, such as automated comparison results and a marking reproduciblity rating, increased support for the ground truth conclusion and could also be a method for providing evidential value to difficult comparisons.
\end{abstract}




\section{Introduction}

The pattern comparison areas of forensic science rely on subjective conclusions made by examiners. While there is a push towards more objective comparison methods, examiners will continue to play a key role in the comparison process. Therefore, it is important to understand their accuracy and error rates, which has been the focus of several previous studies in the field of firearm and toolmark examination [1-10]. However, the forensic science pattern comparison areas continue to be criticized for their subjective nature $[11,12]$. One critical review discussed "foundational validity" in forensic science, defined as showing that a method is repeatable, reproducible, and accurate [12]. While the above listed studies have focused on addressing the accuracy component of foundational validity, much less research has analyzed the reproducibility and repeatability aspects.

Direct comparison between examiners when comparing the same evidence has been studied through previous research. One study used three-dimensional (3D) surfaces and virtual comparison microscopy (VCM) to compare conclusions and determine consensus comparison areas on cartridge cases [13]. The results showed that trained examiners made all correct identifications and eliminations. There were some inconclusive conclusions made on ground truth eliminations, however, these were determined to be due to laboratory policy preventing elimination conclusions in the presence of similar class characteristics. Another study used two-dimensional (2D) images of aperture shear on Glock cartridge cases [14]. These aperture shear images were aligned and displayed to the participants. The authors determined that the examiners in the study reached similar conclusions. One limitation to the two studies just described is that 3D scans [13] and 2D images [14] were used rather than physical cartridge cases. A digital study design has some notable advantages. Once researchers image the cartridge cases of interest, they can easily be shared with all participants. However, examiners do not have the ability to physically examine the items as they do in typical casework. Furthermore, the comparison areas available for the examiners is limited to the surfaces captured during imaging. The VCM study included only the breech face impression area [13], and the Glock aperture shear study only included the aperture shear areas [14].

To complement the above studies, a different study design was implemented that would allow for physical examination of the same cartridge cases by different examiners. A method called double-casting was used to replicate cartridge case test sets [15-17]. These physical samples were then sent to examiners, allowing them to perform comparisons as they would in routine casework. To perform an analysis of the evidential value provided by examiner comparisons, a Bayesian network was used. Bayesian networks have been successfully applied in other fields, such as to analyze customer satisfaction data [18] and risk prediction in the medical field [19]. They have also been proposed for evidence analysis in forensic science [20]. They allow for different data types (nominal, ordinal, and numeric) and present outcomes in probabilistic terms. This flexibility makes 
them suitable for analysis of examiner comparison data because they can easily accommodate the conclusions, automated comparisons results, and any other variables of interest. The focus of this study was on the magnitude of the effects of these variables in combination (e.g. examiner conclusions and automated comparison results) using a likelihood ratio approach.

\section{Methods}

\section{Data collection}

Many studies that have assessed firearm examiner accuracy have used comparison sets made up of multiple cartridge cases fired from the same firearms to send to each participant. While this is a good way to analyze error rates, conclusions from comparison sets between examiners are not directly comparable due to the shot-to-shot variability in markings left on different cartridge cases fired by the same firearms. For that reason, the comparison sets sent out in this study were cartridge case double-casts. The double-casting process has been shown to produce accurate reproductions with low variability [15-17]. An example master cartridge case and double-cast used in this study are shown in Figure 1.

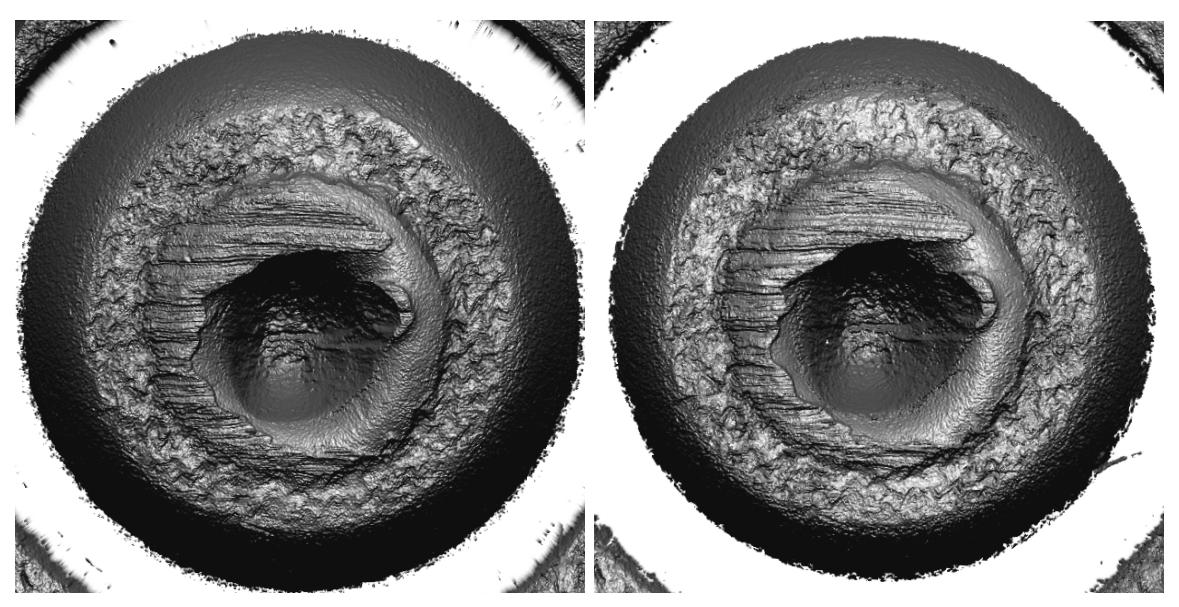

Figure 1: A master cartridge case fired from a Smith \& Wesson SD9VE (left) and its double-cast (right). This master cartridge case was used to make double-casts for the questioned cartridge case in Set 11. Images saved using the Cadre X3P Viewer [21].

Twenty comparison sets of three known double-casts and one unknown double-cast were sent to each of 18 firearm examiners from around the United States that participated in the study. Composition of the test sets is shown in Table 1. Seven of these sets were ground truth identifications and 13 were ground truth eliminations. The sets were imbalanced for two reasons: 1) to prevent examiners from assuming there were an equal number of identification and elimination sets, and 2) because false positives are generally considered more problematic than false negatives, so these 
were tested by including more ground truth elimination sets. An additional set (Set 21) was also sent to the participants, but it was designed in a different way with a different intended analysis. Ten cartridge case double-casts made up that set, and the examiners were asked to determine the number of firearms used to fire the cartridge cases from which the set was composed. Due to the different design and question being asked for Set 21, the analysis in this paper will focus on the first 20 sets.

Table 1: Firearms used to test fire for the known cartridge cases for Sets 1-20. All firearms are chambered in $9 \mathrm{~mm}$ Luger.

\begin{tabular}{lll}
\hline Set Number & Known Firearm & Ground Truth \\
\hline 1 & Taurus 24/7 G2 & Elimination \\
2 & SCCY CPX-2 & Elimination \\
3 & Springfield XD9 & Identification \\
4 & Ruger LC9 & Elimination \\
5 & Hi-Point C9 & Elimination \\
6 & Keltec PF9 & Identification \\
7 & Ruger P95 & Elimination \\
8 & Ruger LC9 & Elimination \\
9 & SCCY CPX-2 & Elimination \\
10 & Hi-Point C9 & Identification \\
11 & Smith \& Wesson SD9VE & Identification \\
12 & Springfield XD9 & Elimination \\
13 & Hi-Point C9 & Elimination \\
14 & Hi-Point C9 & Elimination \\
15 & SCCY CPX-2 & Elimination \\
16 & Taurus 24/7 G2 & Elimination \\
17 & Ruger LC9 & Identification \\
18 & Taurus 24/7 G2 & Identification \\
19 & Springfield XD9 & Elimination \\
20 & SCCY CPX-2 & Identification \\
\hline
\end{tabular}

To develop the master set of cartridge cases, firearms were selected to cover a range of reproduced markings. These firearms included some that left distinct, reproducible markings and some that left markings that were not as reproducible. The ammunition used for all test fires was Federal American Eagle. Prior to double-casting the cartridge cases, a practicing firearm examiner evaluated the sets to ensure they were realistic in terms of the degree and range of difficulty commonly encountered in casework. Twelve of the 13 ground truth elimination sets contained questioned cartridge cases from the same model firearm as the known cartridge cases in an effort to evaluate performance on close non-match comparisons. One ground truth elimination set (Set 7) used a Ruger P95 as the known firearm with the questioned cartridge case fired by a Ruger SR9. 
Although these are different models, class characteristics were similar between these two firearms. Furthermore, this was an open-set design. No indications were given as to the number of ground truth identification and elimination sets, and each set was independent in that the response to one set in no way influenced the responses to other sets

The participants were all mailed the comparison sets (which were made up of double-casts of the master set of fired cartridge cases) and asked to state a conclusion on the source for each set using the AFTE Range of Conclusions [22]. One of the conclusions is "inconclusive" which is broken down into three subcategories [22]:

- Inconclusive A: Some agreement of individual characteristics and all discernible class characteristics, but insufficient for an identification.

- Inconclusive B: Agreement of all discernible class characteristics without agreement or disagreement of individual characteristics due to an absence, insufficiency, or lack of reproducibility.

- Inconclusive C: Agreement of all discernible class characteristics and disagreement of individual characteristics, but insufficient for an elimination.

For each inconclusive conclusion, the examiners were asked to select which subcategory best represented the comparison. The subcategories were then analyzed to extract additional information about the comparisons, rather than simply stating it was inconclusive. Some additional responses were collected about each of the comparisons related to, for example, the difficulty and reproducibility of markings, as well as information about the background and experience of the examiner. The Qualtrics platform was used to digitally record and submit all responses [23]. This is a West Virginia University (WVU) Institutional Review Board (IRB) approved study (protocol \#1812383090).

Automated comparisons were also performed to analyze how examiner conclusions and database results may be complementary. All master cartridge cases were scanned with a Sensofar S neox 3D optical profiler using the "Confocal Fusion" scan mode and a lateral resolution of $1.38 \mu \mathrm{m}$ per pixel. Confocal fusion is a proprietary method developed by Sensofar to combine the strengths of confocal microscopy and focus variation. This method attempts to measure a confocal data point at each location of the cartridge case surface. If this measurement is unsuccessful, a focus variation measurement is attempted instead. The goal is to combine the strengths of both methods: better resolution with confocal microscopy and the ability to measure steeper sloping areas with focus variation. The scans were saved in the *.x3p file format for software interoperability [24]. The National Institute of Standards and Technology (NIST) toolmark comparison algorithms were applied for objective comparison of the cartridge cases within each set through an ongoing 
collaboration. The known cartridge cases within each set were pairwise-compared to each other, and the questioned cartridge cases were compared to each known to mimic how evidence should be analyzed in casework [25].

Data pre-processing for automated comparison included trimming the surfaces to include only the breech face area. This included removing any flowback. A robust bandpass filter was applied to each measured surface to attenuate noise, form, and waviness with $25 \mu \mathrm{m}$ and $400 \mu \mathrm{m}$ cutoff wavelengths. Prior to comparison, the surfaces were downsampled to $2.76 \mu \mathrm{m}$ per pixel to decrease the comparison time without sacrificing comparison accuracy. Parameters of the CMC algorithm included $500 \times 500 \mu \mathrm{m}$ cells in an approximately $8 \times 8$ grid, depending on the size of the cropped area, to cover the breech face impression area. Each cell was searched over the comparison cartridge case to locate the region of highest similarity. If the cell was within preset tolerances in terms of similarity, position, and rotation the cell was labeled as congruent. The cell congruency thresholds were $20 \%$ similarity, $125 \mu \mathrm{m}$ in $\mathrm{x}$ and $\mathrm{y}$ spatial positioning, and $3^{\circ}$ in rotation. The output of the algorithms included two metrics: the areal correlation coefficient (ACCF) corresponding to the overall similarity between the two surfaces and the number of congruent matching cells (CMCs). The interested reader may refer to the NIST CMC publications for more detail [26-28].

\section{Bayesian networks}

Bayes' theorem relates the probabilities before analysis of the evidence in a case to the probabilities after the evidence is analyzed, as shown in Equation 1. As an example, a cartridge case is recovered at a crime scene and a suspect firearm has also been located and submitted to a firearms examiner. The examiner conducts test fires of the suspect firearm for comparison to the crime scene cartridge case. The comparison results correspond to the evidence $E$ in the case. The evidence can then be interpreted in terms of the prosecutorial hypothesis $\left(\theta_{p}\right)$ that the suspect firearm discharged the questioned cartridge case, and in terms of the defense hypothesis $\left(\theta_{d}\right)$ that the questioned cartridge case was fired by some other firearm. This analysis makes up the boxed term in Equation 1. This term is the likelihood ratio (LR) and corresponds to analysis of the evidence under the two competing hypotheses. The LR relates the prior probabilities to the posterior probabilities. The prior probabilities $\left(P\left(\theta_{p}\right)\right.$ and $\left.P\left(\theta_{d}\right)\right)$ are not conditioned on the evidence and represent previous knowledge about the case before analysis of the evidence. These are typically not in the domain of the forensic scientist and are therefore assessed by the fact finder. The LR can then be used to update the prior probabilities to the posterior probabilities $\left(P\left(\theta_{p} \mid E\right)\right.$ and $\left.P\left(\theta_{d} \mid E\right)\right)$. This equates to the prior probability that the suspect firearm discharged the questioned cartridge case, multiplied by the weight of the evidence (the LR), resulting in the posterior probability that the suspect firearm discharged the questioned cartridge case given the evidence. Because the posterior probabilities require knowing the prior probabilities they are also in the domain of the fact finder and not the 
forensic scientist. I represents any additional information needed for each respective probability, however, it has been and will be removed from all notation other than Equation 1 for conciseness.

$$
\frac{P\left(\theta_{p} \mid I\right)}{P\left(\theta_{d} \mid I\right)} \times \frac{P\left(E \mid \theta_{p}, I\right)}{P\left(E \mid \theta_{d}, I\right)}=\frac{P\left(\theta_{p} \mid E, I\right)}{P\left(\theta_{d} \mid E, I\right)}
$$

A Bayesian network can be used to directly assess the factors related to Bayes' theorem (Equation 1). A Bayesian network is a graphical representation of a set of variables and their conditional dependencies. Each node represents a variable, and the connections drawn between them, called arcs, define their conditional probability distributions. Using Figure 2 as an example, the arc drawn from node $C$ to node $X$ defines the conditional probability distribution of node $X$ to be $P(X \mid C)$. The value taken by $X$ is dependent on the value taken by $C$. In this example, $C$ is said to be the parent of $X$, and likewise, $X$ is the child of $C$. Nodes may have multiple parents and/or children, as well as multiple incoming and/or outgoing arcs, and the combination of all nodes and arcs defines the network. The full joint probability distribution between all of the variables is simplified using a Bayesian network because it can be represented by the product of each local conditional probability distribution. As described above, the local conditional probability distributions only depend on each node's immediate parents. Efficient inference is then able to be conducted.

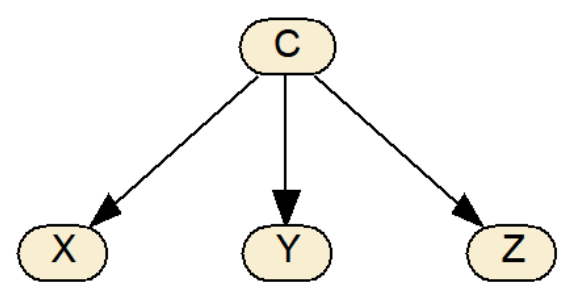

Figure 2: Example network.

One of the challenges with Bayesian networks is defining the model structure. One way to determine the structure is through expert knowledge about relationships between variables. Using this method, an expert creates all necessary arcs in the network. The parameters of the network are learned simply through counting variable frequencies, given the structure, based on a complete data file. The result is a complete network structure with conditional probability tables for each node. The Bayesian network software Netica was used in this study to manually create a network [29]. The RNetica package was also used to allow for networks to be created and evaluated using the $\mathrm{R}$ programming language [30].

A second approach to building the model structure is using one of many available algorithms [31]. One such algorithm used in this study was tree augmented naive Bayes (TAN). To understand this algorithm, a brief background on naive Bayes is necessary. In the naive Bayes method, one 
variable is selected as the class variable. There is a strong independence assumption between all remaining variables, where each variable is only allowed to be connected to the class variable [32]. No other relationships are modeled. The example network shown in Figure 2 is a naive Bayes model, where node $C$ is the class variable and nodes $X, Y$, and $Z$ are the other variables, conditionally independent of each other given node $C$. While it has been shown to perform well in classification tasks, the independence assumption may be unrealistic [31]. The TAN algorithm is similar to naive Bayes but the strong independence assumption is relaxed [32]. Defining a class variable is still required. Every other variable is connected to the class variable but can have one other parent. Mutual information (MI), described in the next section, is used to determine the second connection for each variable. This method is known as semi-naive Bayesian learning and is still somewhat restricted, but it allows for modeling some of the interdependencies within the data that are not possible with naive Bayes [33]. The parameters of the model are learned using counting in the same way as the manual method described above. The TAN algorithm is currently implemented in Netica [29].

The PC algorithm is another automated approach for determining the model structure [31, 34]. This algorithm is based on a series of $\chi^{2}$ statistical dependence tests to make connections between variables. The arcs are then directed either randomly or using expert knowledge [31]. Netica currently does not implement the PC algorithm, so the GeNIe software was used for this purpose instead [35]. For the data used in this study, the PC algorithm resulted in 195 node connections, compared with 65 for the manual network and 59 for the TAN network, making the conditional probability tables too large to learn. RNetica stated that 131.3 gigabytes (GB) of memory were needed. For efficiency concerns, this method was not utilized any further and the analysis continued with the manual and TAN networks.

Two Bayesian networks were created from the examiner data, and each of the 31 nodes are defined in Table 2. Going forward, notation will include bold words for node titles and italicized words for node states. Generally, the node states were pre-determined categories for the examiners to select from during the study. For example, the five states in the Comparison_time node were those that were presented as options for each comparison. Exceptions to this would include the Set_number and Firearm_ID nodes as they were defined by the study design. The ACCF and CMC node states were determined by the results from the automated comparisons. 
Table 2: Definitions for all nodes in the Bayesian network.

\begin{tabular}{|c|c|}
\hline Node & Description \\
\hline ACCF_interpretation & $\begin{array}{l}\text { Overall classification from the CMC algorithm. Same firearm is defined as any } \\
\text { comparison (Q-K1, Q-K2, Q-K3) with greater than or equal to } 30 \% \text {. This } \\
\text { threshold was based on in-house testing of the algorithm. }\end{array}$ \\
\hline ACCF_K1Q & Overall similarity of the comparison of Q to $\mathrm{K} 1$ from the $\mathrm{CMC}$ algorithm. \\
\hline ACCF_K2Q & Overall similarity of the comparison of $\mathrm{Q}$ to $\mathrm{K} 2$ from the $\mathrm{CMC}$ algorithm. \\
\hline ACCF_K3Q & Overall similarity of the comparison of Q to $\mathrm{K} 3$ from the CMC algorithm. \\
\hline CMC_interpretation & $\begin{array}{l}\text { Congruent cell classification from the CMC algorithm. Same firearm is defined as } \\
\text { any comparison (Q-K1, Q-K2, Q-K3) with greater than or equal to six congruent } \\
\text { cells. This threshold was based on in-house testing, as well as research published } \\
\text { by NIST [26-28]. }\end{array}$ \\
\hline CMC_K1Q & Congruent cell counts of the comparison of Q to $\mathrm{K} 1$ from the CMC algorithm. \\
\hline CMC_K2Q & Congruent cell counts of the comparison of $\mathrm{Q}$ to $\mathrm{K} 2$ from the $\mathrm{CMC}$ algorithm. \\
\hline CMC_K3Q & Congruent cell counts of the comparison of Q to $\mathrm{K} 3$ from the CMC algorithm. \\
\hline Comp_other_evidence & Displays if the participant is responsible for comparisons other than firearms. \\
\hline Comparison_area_BF & Use of the breech face impression area for comparison. \\
\hline Comparison_area_FP & Use of the firing pin impression area for comparison. \\
\hline Comparison_time & Approximate time spent on each comparison. \\
\hline Cont_education_freq_year & $\begin{array}{l}\text { The number of continue education courses, workshops, etc. attended by each participant. } \\
\text { Combination of the ACCF and CMC data. Same firearm is defined as the ACCF and }\end{array}$ \\
\hline Database_interpretation & $\begin{array}{l}\text { CMC interpretation nodes both indicating same firearm based on their respective } \\
\text { thresholds ( } 30 \% \text { and } 6 \text { cells, respectively). If results conflict, CMC interpretation is used. }\end{array}$ \\
\hline Difficulty_comparison & Comparison difficulty of each set rated by each participant. \\
\hline Firearm_cases_worked & The approximate number of firearms cases worked per year. \\
\hline Firearm_ID & Unique firearm identifier used as the sample firearms. \\
\hline Firearms_experience & The amount of time each participant has been a firearm examiner. \\
\hline Ground_truth & The ground truth of each comparison set. \\
\hline Highest_degree & The highest degree held by each participant. \\
\hline Identifier & Unique string to identify each participant. \\
\hline Last_proficiency_test & Time frame for completion of last proficiency test in firearms. \\
\hline More_knowns & Yes if the participant would have performed additional test fires for a comparison. \\
\hline Participant_conclusion & $\begin{array}{l}\text { The conclusions made by each participant for each set. Follows the } \\
\text { AFTE Range of Conclusions [22]. }\end{array}$ \\
\hline Perform_research & Yes if the participant has done firearms research. \\
\hline Published & Yes if the participant has publications in journals, books, etc. \\
\hline Reproducibility & Rating for the reproducibility of markings on the known cartridge cases by each participant for each set. \\
\hline Set_number & Number defining the comparison sets used in the study. \\
\hline Subclass & Yes if subclass characteristics were observed on any of the cartridge cases for a given set. \\
\hline Teach_training & Yes if the participant has taught any training courses. \\
\hline Times_testify & The approximate number of times each participant testifies per year. \\
\hline
\end{tabular}

\section{Sensitivity to findings}

In a Bayesian network it may be of interest to determine the sensitivity of a target node to findings at other nodes. In terms of the examiner network, it is useful to know which nodes are most informative in reducing the uncertainty of the ground truth of a given comparison. This will inform which variables in the network are most relevant in predicting the ground truth. The sensitivity measure used here and shown in Equation 2 is known as mutual information (MI) and relates to the reduction in uncertainty of a target node due to knowing the value of any other node [36]. In Equation 2, $P(x, y)$ represents the sensitivity of node $X$ to some other node $Y$ and is calculated by instantiating node $Y$ to each of its values and computing the resulting $P(x \mid y)$. The priors are 
represented by $P(x)$ and $P(y)$. This is translated into a single summary value through summation which is the MI. Netica directly implements the MI calculation. Recall that the TAN algorithm utilizes the MI in determining node connections. For the TAN algorithm, node pairs $X$ and $Y$ are evaluated given node $C$ (the pre-selected class variable), that is $M I(X, Y \mid C)$. Connections are made between the node pairs that are most informative.

$$
M I(X, Y)=\sum_{x, y} P(x, y) \log \frac{P(x, y)}{P(x) P(y)}
$$

\section{Model performance}

Leave-one-out cross validation (LOOCV) was performed to analyze performance of each of the two network structures. One observation was removed from the data file of examiner conclusions, the network was built and trained on the remaining observations, and the network was tested on the observation which was removed. This was repeated for every observation in the data set using both networks. To analyze the LOOCV results, receiver operating characteristic (ROC) curves were used [37, 38]. A ROC curve plots the true positive rate against the false positive rate. The area under the curve (AUC) generally ranges from 0.5 to 1.0 and corresponds to the performance of the classifier. It is defined as the probability that a randomly selected positive instance is ranked higher than a randomly selected negative instance.

As a baseline of performance, only the participant conclusions were initially used to predict classification of each test observation. In an effort to improve the classification ability of each network, a metric was needed to determine which nodes were likely to be the most beneficial. The MI informed which node combinations were tested. The classification of each tested observation was based on which ground truth conclusion was most likely based on the network probabilities of the ground truth node. ROC curves and their respective AUC values were calculated. The $\mathrm{pROC} R$ package was used for statistical comparison of the ROC curves [39].

\section{Likelihood ratio magnitudes}

LRs were extracted from the Bayesian network using Equation 3, a simple re-arrangement of Bayes' theorem. These LRs were calculated using different combinations of variables (Table 2) based on the mutual information results (Equation 2). The goal was to determine what effect these additional variables had on the LRs produced compared to just the examiner conclusions.

$$
L R=\frac{P\left(\theta_{p} \mid E\right) \times P\left(\theta_{d}\right)}{P\left(\theta_{d} \mid E\right) \times P\left(\theta_{p}\right)}
$$


Before continuing, a note must be made in reference to one participant. This participant chose to withdraw from the study after completing 20 of the comparison sets, and five false positives were made by this participant (Sets 2, 7, 9, 14, and 16). Due to the decision to withdraw, these conclusions were not included in the data analysis below but are included here for completeness in accordance with federal research guidelines [40].

\section{Results and Discussion}

\section{Overall study data}

Table 3 displays the overall study results from the 17 participating examiners. The three inconclusive category headings are defined as follows: a "true inconclusive" was all inconclusive B conclusions, a "correct inconclusive" was an inconclusive A on a ground truth identification or an inconclusive $\mathrm{C}$ on a ground truth elimination, and an "incorrect inconclusive" was an inconclusive $\mathrm{A}$ on a ground truth elimination or an inconclusive $\mathrm{C}$ on a ground truth identification (the inconclusive A, B, and C subcategories have been previously defined above based on the AFTE Range of Conclusions [22]). There was one false positive made in the study on Set 16, however, no other participants made the same error. Furthermore, there were no false negatives.

Table 3: Examiner conclusions across the 20 test sets.

\begin{tabular}{llllllll}
\hline Test Set & True & True & True & Correct & Incorrect & False & False \\
& Positives & Negatives & Inconclusives & Inconclusives & Inconclusives & Positives & Negatives \\
\hline Set 1 & 0 & 14 & 1 & 1 & 1 & 0 & 0 \\
Set 2 & 0 & 9 & 3 & 4 & 1 & 0 & 0 \\
Set 3 & 17 & 0 & 0 & 0 & 0 & 0 & 0 \\
Set 4 & 0 & 12 & 2 & 3 & 0 & 0 & 0 \\
Set 5 & 0 & 13 & 1 & 2 & 1 & 0 & 0 \\
Set 6 & 17 & 0 & 0 & 0 & 0 & 0 & 0 \\
Set 7 & 0 & 13 & 2 & 2 & 0 & 0 & 0 \\
Set 8 & 0 & 14 & 3 & 0 & 0 & 0 & 0 \\
Set 9 & 0 & 10 & 3 & 3 & 1 & 0 & 0 \\
Set 10 & 17 & 0 & 0 & 0 & 0 & 0 & 0 \\
Set 11 & 17 & 0 & 0 & 0 & 0 & 0 & 0 \\
Set 12 & 0 & 12 & 1 & 3 & 1 & 0 & 0 \\
Set 13 & 0 & 12 & 1 & 3 & 1 & 0 & 0 \\
Set 14 & 0 & 11 & 1 & 4 & 1 & 0 & 0 \\
Set 15 & 0 & 10 & 2 & 2 & 3 & 0 & 0 \\
Set 16 & 0 & 13 & 1 & 2 & 0 & 1 & 0 \\
Set 17 & 2 & 0 & 13 & 2 & 0 & 0 & 0 \\
Set 18 & 15 & 0 & 1 & 1 & 0 & 0 & 0 \\
Set 19 & 0 & 12 & 1 & 3 & 1 & 0 & 0 \\
Set 20 & 16 & 0 & 1 & 0 & 0 & 0 & 0 \\
\hline
\end{tabular}




\section{Eliciting the model structure}

The first structure was determined manually using knowledge about the variables to make the node connections and is displayed in Figure 3. As an example of two connected variables, there is an arc drawn from Reproducibility to Difficulty_comparison. This reads as the probability of the comparison difficulty given the reproducibility of the markings on the cartridge cases. It is expected that when Reproducibility $=$ Poor, the comparison would be more difficult than when Reproducibility = Excellent . The network structure reflects this relationship which was observed in the data. The highest probability of Difficulty_comparison = Very_difficult was when Reproducibility $=$ Poor . Likewise, the highest probability of Difficulty_comparison = Very_easy was when Reproducibility $=$ Excellent .

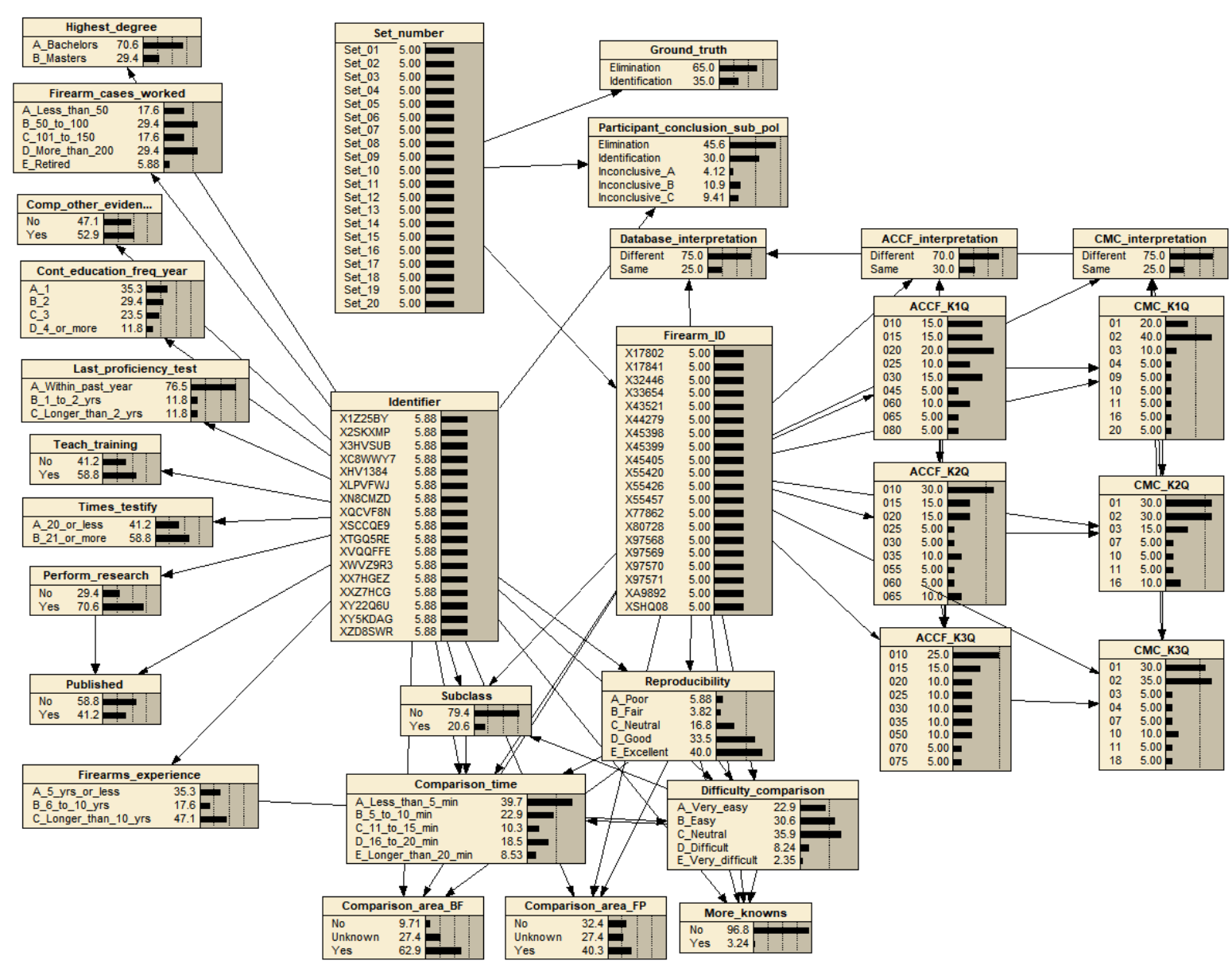

Figure 3: Bayesian network created from the examiner comparison results using expert knowledge.

The second structure, illustrated in Figure 4, was created by the TAN algorithm. There is one connection from Ground_truth to every other variable, and every other variable is allowed one other parent as determined by the MI metric. In this network, Reproducibility and Diffi- 
culty_comparison are both connected to the Ground_truth node, the class variable required by the TAN algorithm, and the Identifier node. This makes sense as each participant rated the reproducibility of the known cartridge cases and the comparison difficulty for each set, however, any other relationships with these two variables are not able to be represented.

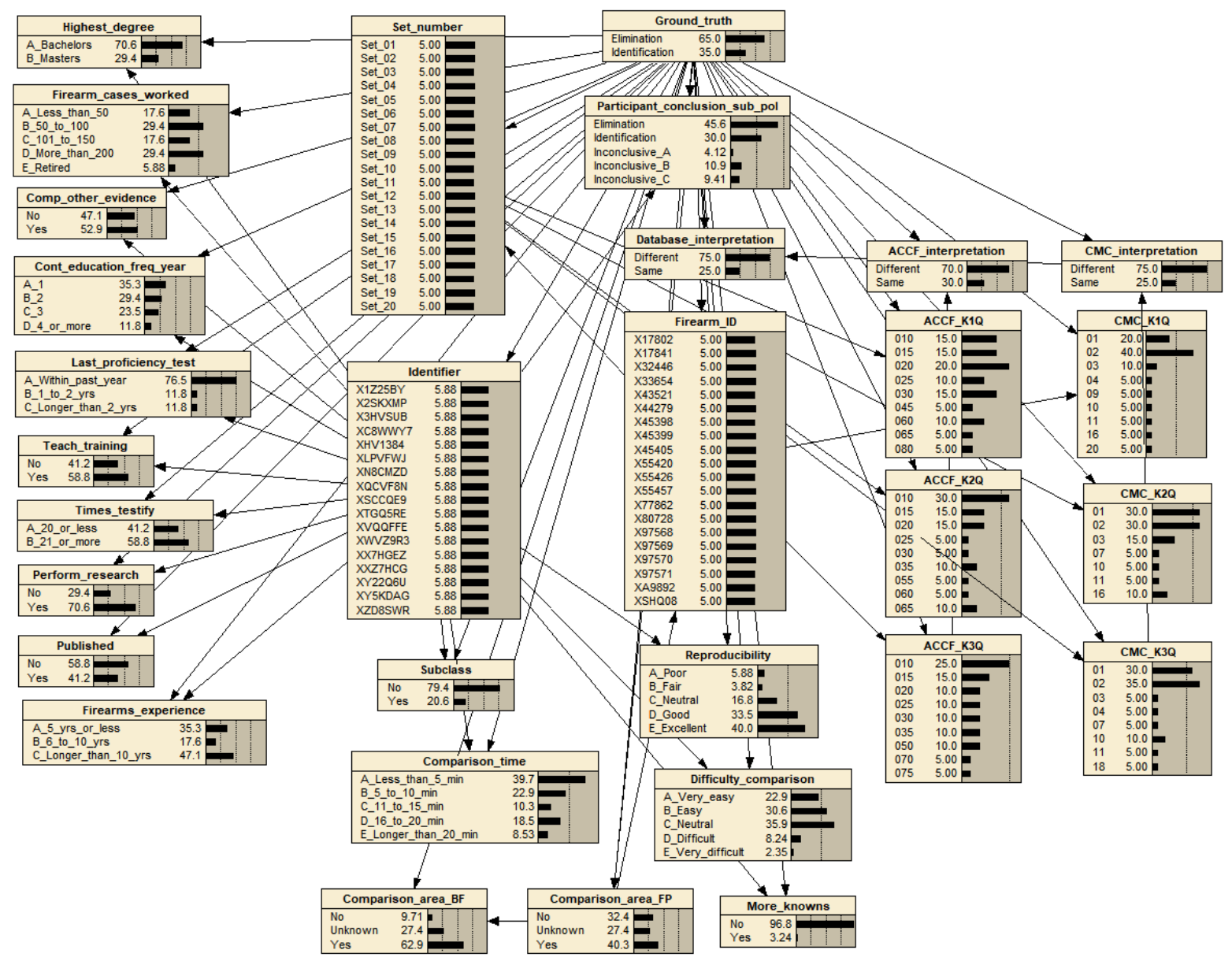

Figure 4: Bayesian network created from the examiner comparison results using the TAN algorithm.

The values currently displayed in the networks are the prior probabilities for each node and are equal for both networks due to the same data being used. For example, $65 \%$ of the comparison sets were ground truth eliminations (13/20) while 35\% (7/20) were ground truth identifications. These values are reflected by the Ground_truth node in each network.

\section{Node sensitivity}

To determine the most informative variables for each network, the MI metric was applied. Table 4 displays the MI values when the Ground_truth node is the target node and no findings have been 
entered into the network. The MI values in this scenario are identical between the two network structures. The MI, in bits of information, is displayed for each node along with a percent. The percent is scaling the MI for a given node to the maximum shown in the first row of the table. It is obvious that knowing the value of Ground_truth will lead to a $100 \%$ reduction in uncertainty about the ground truth. Furthermore, Set_number and Firearm_ID will also lead to a $100 \%$ reduction in uncertainty because each set has a single ground truth and each set has a unique firearm, so these rows may be disregarded. The nodes that corresponded to the individual ACCF and CMC scores were removed from this analysis. The known cartridge cases were arbitrarily numbered one, two, and three, and therefore there was no meaning associated with those labels. Furthermore, an examiner is unlikely to only consider one comparison score and instead will consider the results from all three comparisons ( $\mathrm{Q}$ to $\mathrm{K} 1, \mathrm{~K} 2$, and K3). Participant_conclusion is therefore the most informative node and is providing an $82.8 \%$ reduction in uncertainty. The remaining nodes in the network not listed in the table provide no information towards the true value of the Ground truth node.

Table 4: Sensitivity of Ground truth to findings at other nodes.

\begin{tabular}{lll}
\hline Node & Mutual Information & Percent \\
\hline Ground_truth & 0.93407 & 100 \\
Set_number & 0.93406 & 100 \\
Firearm_ID & 0.93396 & 100 \\
Participant_conclusion & 0.77318 & 82.8 \\
ACCF_interpretation & 0.67406 & 72.2 \\
Database_interpretation & 0.50904 & 54.5 \\
CMC_interpretation & 0.509 & 54.5 \\
Comparison_area_BF & 0.06301 & 6.75 \\
Comparison_area_FP & 0.05959 & 6.38 \\
Difficulty_comparison & 0.01548 & 1.66 \\
Reproducibility & 0.00962 & 1.03 \\
More_knowns & 0.00112 & 0.12 \\
Comparison_time & 0.0004 & 0.0426 \\
Subclass & 0.00038 & 0.0407 \\
\hline
\end{tabular}

The sensitivity analysis takes into account the findings at all query nodes, meaning that as findings are added into other nodes in the network the MI values will change. This is useful if a finding is entered in for a particular node and it is necessary to know which node is likely to be the next most helpful in reducing the uncertainty about the target node given previous findings. If the Participant_conclusion node is instantiated, the sensitivity analysis on the Ground_truth node will show which node should be instantiated next to provide the largest reduction in uncertainty 
given the conclusion selected in the Participant_conclusion node. The node sensitivities will change depending on which of the five conclusions are selected.

MI results are summarized in Table 5 for both network structures. Participant_conclusion was always the first node because of the results shown in Table 4. As an example using Table 5, for the manual network when Participant_conclusion = Identification, ACCF interpretation is the next most informative node. However, no third node is needed due to little additional information being provided by any node after ACCF_interpretation. When Participant_conclusion $=$ Inconclusive $A$, Reproducibility is the next most informative node. The state selected for Reproducibility determines whether it is a terminal node or if a third node is useful. For example, when Reproducibility $=$ Poor, no third node is needed, but when Reproducibility $=$ Fair the ACCF_interpretation node provides additional information towards the Ground_truth node.

Table 5: MI results for each of the two network structures.

\begin{tabular}{|c|c|c|c|}
\hline Network & First Node & Second Node & Third Node \\
\hline Manual & $\begin{array}{l}\text { Participant_conclusion_sub_pol }=\text { Identification } \\
\text { Participant_conclusion_sub_pol }=\text { Inconclusive_A } \\
\text { Participant_conclusion_sub_pol }=\text { Inconclusive_A } \\
\text { Participant_conclusion_sub_pol }=\text { Inconclusive_A } \\
\text { Participant_conclusion_sub_pol = Inconclusive_A } \\
\text { Participant_conclusion_sub_pol = Inconclusive_A } \\
\text { Participant_conclusion_sub_pol = Inconclusive_B } \\
\text { Participant_conclusion_sub_pol = Inconclusive_B } \\
\text { Participant_conclusion_sub_pol = Inconclusive_B } \\
\text { Participant_conclusion_sub_pol = Inconclusive_B } \\
\text { Participant_conclusion_sub_pol = Inconclusive_B } \\
\text { Participant_conclusion_sub_pol = Inconclusive_C } \\
\text { Participant_conclusion_sub_pol = Elimination }\end{array}$ & $\begin{array}{l}\text { ACCF_interpretation } \\
\text { Reproducibility }=\text { Poor } \\
\text { Reproducibility }=\text { Fair } \\
\text { Reproducibility }=\text { Neutral } \\
\text { Reproducibility }=\text { Good } \\
\text { Reproducibility }=\text { Excellent } \\
\text { Reproducibility }=\text { Poor } \\
\text { Reproducibility }=\text { Fair } \\
\text { Reproducibility }=\text { Neutral } \\
\text { Reproducibility }=\text { Good } \\
\text { Reproducibility }=\text { Excellent }\end{array}$ & $\begin{array}{l}\text { ACCF_interpretation } \\
\text { More_knowns } \\
\text { Comparison_time } \\
\text { ACCF_interpretation }\end{array}$ \\
\hline TAN & $\begin{array}{l}\text { Participant_conclusion_sub_pol }=\text { Identification } \\
\text { Participant_conclusion_sub_pol }=\text { Inconclusive_A } \\
\text { Participant_conclusion_sub_pol }=\text { Inconclusive_A } \\
\text { Participant_conclusion_sub_pol }=\text { Inconclusive_A } \\
\text { Participant_conclusion_sub_pol }=\text { Inconclusive_B } \\
\text { Participant_conclusion_sub_pol }=\text { Inconclusive_B } \\
\text { Participant_conclusion_sub_pol }=\text { Inconclusive_B } \\
\text { Participant_conclusion_sub_pol }=\text { Inconclusive_B } \\
\text { Participant_conclusion_sub_pol }=\text { Inconclusive_C } \\
\text { Participant_conclusion_sub_pol }=\text { Elimination }\end{array}$ & $\begin{array}{l}\text { ACCF_interpretation } \\
\text { Last_proficiency_test }=\text { Within_past_year } \\
\text { Last_proficiency_test }=1 \text { _to_2_years } \\
\text { Last_proficiency_test }=\text { Longer_than_2_years } \\
\text { Cont_education_freq_year }=1 \\
\text { Cont_education_freq_year }=2 \\
\text { Cont_education_freq_year }=3 \\
\text { Cont_education_freq_year }=4 \text { _or_more }\end{array}$ & $\begin{array}{l}\text { Firearm_cases_worked } \\
\text { Firearm_cases_worked } \\
\text { Firearms_experience } \\
\text { ACCF_interpretation }\end{array}$ \\
\hline
\end{tabular}

The two network structures, manual and TAN, show some similarities in the MI results. When Participant_conclusion = Identification, the second node for both structures is ACCF interpretation. For both Participant_conclusion = Elimination and Participant_conclusion $=$ Inconclusive_C , no second nodes are needed. The differences occur with Participant_conclusion $=$ Inconclusive $A$ and Participant $\_$conclusion $=$Inconclusive $B$.

The MI results just described were used to select additional nodes in an effort to improve LOOCV classification. These tests for both networks were defined as: 
- Test 1: Only the Participant Conclusion was used for classification.

- Test 2: The ACCF results were combined with the Participant Conclusion.

- Test 3: The CMC results were combined with the Participant Conclusion.

- Test 4: The ACCF and CMC results were both combined with the Participant Conclusion.

- Test 5: The ACCF and Reproducibility were combined with the Participant Conclusion as these were the most informative second nodes for the manual network.

- Test 6: The ACCF, Last Proficiency Test, and Continuing Education Frequency per Year were combined with the Participant Conclusion as these were the most informative second nodes for the TAN network.

Due to the changing nature of the MI under different conditions, it was unknown if a single combination would produce ideal results for every comparison. For this reason an adaptive approach was adopted. Rather than using the same nodes in all circumstances, the nodes utilized were dynamically selected based on the MI results. For example using the manual network, if the Participant_conclusion was Elimination, then only the Participant_conclusion node was used to predict the ground truth. However, if the Participant_conclusion was Inconclusive_B then Reproducibility was also used, and the Reproducibility state selected was then used to determine which next node to use for prediction of that test case following the guide in Table 5. The idea was to try to maximize performance utilizing a data-driven approach. This approach was used as a seventh test for each network.

\section{Model performance comparison}

The ROC curve AUC values from the seven LOOCV tests of each network structure are displayed in Table 6. The Participant_conclusion and Participant_conclusion with ACCF_interpretation and $\mathbf{C M C}$ interpretation combinations resulted in the same AUC values for both networks. For the manual structure, the Participant_conclusion had the lowest AUC while the Participant_conclusion - ACCF interpretation - Reproducibility combination had the highest. The TAN network structure resulted in the same lowest and highest test node AUC values. This is fortuitous because it lends support that practical and statistical linkages overlap.

The Participant_conclusion - ACCF interpretation combination resulted in a larger AUC than the Participant_conclusion - CMC_interpretation combination. Both the ACCF and CMC metrics performed poorly on Set 17, but the CMC metric also did not perform well on Set 18 . This led to additional test observations incorrectly classified when CMC was used without the ACCF. Further proof of this is the larger AUC when the ACCF and CMC metrics were considered together 
Table 6: ROC AUC values for leave-one-out cross validation. The test nodes were used to provide information towards the ground truth.

\begin{tabular}{lll}
\hline Test & Manual AUC & TAN AUC \\
\hline Conclusion & 0.9792 & 0.9792 \\
Conclusion, ACCF & 0.9859 & 0.9859 \\
Conclusion, CMC & 0.9832 & 0.9832 \\
Conclusion, ACCF, CMC & 0.9859 & 0.9859 \\
Conclusion, ACCF, Reproducibility & 0.9989 & 0.9950 \\
Conclusion, ACCF, Proficiency, Continuing Education & 0.9955 & 0.9924 \\
Adaptive & 0.9996 & 0.9904 \\
\hline
\end{tabular}

with the Participant_conclusion node, although the AUC was equal to when only the ACCF was used. This led to the ACCF being part of the remaining test combinations instead of the CMC metric.

There were two misclassifications for the manual network adaptive approach. One was the false positive conclusion. The other was an inconclusive B conclusion on a ground truth elimination. Following the guide in Table 5, Reproducibility was used which was Neutral, leading to Comparison_time which was 16 to 20 minutes. This combination led to a predicted identification which was incorrect.

The ROC curves were used to compare performance between the two network structures using the $\mathrm{pROC} R$ package [39]. Because the first four rows in Table 6 resulted in the same AUC values, these were not statistically compared as they would result in a $p$-value of 1.000 indicating no significant differences. The test node combinations from the last three rows in Table 6 were statistically compared at the 0.05 level of significance. The resulting $p$-values were $0.036,0.018$, and 0.006, respectively. A Bonferroni correction was applied to the $\alpha$ value to account for the three statistical comparisons performed. The new $\alpha$ value was 0.016 . Comparing each $p$-value to 0.016 led to a single significant difference which was between the adaptive approaches.

While the two networks performed similarly, the adaptive approach performed significantly better with the manual network than with the TAN network. Furthermore, additional variable relationships were modeled with the manual network that were not possible with the TAN network due to its restricted nature. For these reasons, the manual network was selected for further analysis.

\section{Likelihood ratio magnitude results}

The purpose of the results discussed in the previous sections was to analyze the ability of the network to classify comparison results. This performance was indicated through use of ROC curve AUC values using node combinations informed by MI. After identifying performance of the net- 
works, LRs were evaluated using the manually created network structure. Based on a leave-oneexaminer-out approach, a LR was calculated for each comparison set based on the participant conclusion along with the node combinations identified through the MI results discussed above. All LRs are represented as $\log _{10}$ likelihood ratios (LLRs) to make the magnitudes symmetric around zero and not have the $H_{d}$ results bounded between zero and one.

Table 7 displays the mean LLRs across all 17 examiners for each set based on their conclusions. Overall, the LLRs are indicative of the true proposition, meaning the values are negative for all ground truth elimination comparisons and positive for all ground truth identification comparisons. Set 17 resulted in a less conclusive LLR at 0.3. While positive indicating a ground truth identification, it is close to zero which would not provide support for either proposition. This result makes sense given that 13 of 17 participants selected an "inconclusive" conclusion for the Set 17 comparison.

Table 7: LLRs calculated from using only the participant conclusions.

\begin{tabular}{llr}
\hline Set & Ground Truth & LLR \\
\hline 1 & Elimination & -4.1 \\
2 & Elimination & -3.4 \\
3 & Identification & 2.4 \\
4 & Elimination & -4.1 \\
5 & Elimination & -4.1 \\
6 & Identification & 2.4 \\
7 & Elimination & -4.1 \\
8 & Elimination & -3.9 \\
9 & Elimination & -3.5 \\
10 & Identification & 2.4 \\
11 & Identification & 2.4 \\
12 & Elimination & -4.1 \\
13 & Elimination & -4.1 \\
14 & Elimination & -4.0 \\
15 & Elimination & -3.3 \\
16 & Elimination & -3.8 \\
17 & Identification & 0.3 \\
18 & Identification & 2.1 \\
19 & Elimination & -4.1 \\
20 & Identification & 2.3 \\
\hline
\end{tabular}

Table 8 displays the mean change in LLR for the set numbers and node combinations as compared to the base LLRs in Table 7. The signs indicate which direction the LLR is shifting. For example, a negative LLR in Table 8 indicates the LLR is getting smaller, and for a ground truth elimination the LLR is now showing stronger support for an elimination. The goal was to deter- 
mine if other variables were able to benefit the comparison process and support examiner conclusions. As can be seen by looking at the magnitudes, the ground truth elimination sets generally change less than the ground truth identification sets. This is because there were no false negatives in this study, meaning the participant conclusion was already very indicative of the ground truth leaving little room for adjustments with additional variables. The ground truth identification set LLRs, other than Set 17, show an increase in the LLR by three to four orders of magnitude. This is because of the false positive in the study which led to lower LLRs supporting the ground truth identifications, allowing additional variables more power in supplementing the examiner conclusions.

Table 8: Mean change in LLR magnitude considering the results from each examiner for the provided set and node combinations.

\begin{tabular}{llrrrrrr}
\hline Set & Ground Truth & ACCF & CMC & ACCF and CMC & $\begin{array}{l}\text { ACCF and } \\
\text { Reproducibility }\end{array}$ & $\begin{array}{l}\text { ACCF and Proficiency and } \\
\text { Continuing Education Frequency }\end{array}$ & Adaptive \\
\hline 1 & Elimination & -0.6 & -0.4 & -0.6 & -1.4 & -0.7 & -1.0 \\
2 & Elimination & -0.6 & -0.4 & -0.6 & -1.9 & -0.7 & -1.4 \\
3 & Identification & +4.0 & +3.9 & +4.0 & +4.0 & +4.1 & +4.0 \\
4 & Elimination & -0.6 & -0.4 & -0.6 & -0.0 & -0.4 & -0.4 \\
5 & Elimination & -0.6 & -0.4 & -0.6 & -1.4 & -0.7 & -0.7 \\
6 & Identification & +4.0 & +3.9 & +4.0 & +4.0 & +4.1 & +4.0 \\
7 & Elimination & -0.6 & -0.4 & -0.6 & -1.1 & -0.7 & -0.7 \\
8 & Elimination & -0.6 & -0.4 & -0.6 & -1.1 & -0.3 & -1.0 \\
9 & Elimination & -0.6 & -0.4 & -0.6 & -1.6 & -0.3 & -0.9 \\
10 & Identification & +4.0 & +3.9 & +4.0 & +4.0 & +4.1 & +4.0 \\
11 & Identification & +4.0 & +3.9 & +4.0 & +4.0 & +4.1 & +4.0 \\
12 & Elimination & -0.6 & -0.4 & -0.6 & -1.3 & -0.7 & -0.9 \\
13 & Elimination & -0.6 & -0.4 & -0.6 & -1.3 & -0.5 & -0.7 \\
14 & Elimination & -0.6 & -0.4 & -0.6 & -1.4 & -0.5 & -0.7 \\
15 & Elimination & -0.5 & -0.3 & -0.5 & -2.0 & -1.5 \\
16 & Elimination & -0.7 & -0.5 & -0.7 & -1.4 & +0.8 & -0.8 \\
17 & Identification & -0.3 & -0.1 & -0.3 & +0.6 & +0.1 & +3.0 \\
18 & Identification & +3.8 & -0.7 & +3.5 & +3.9 & -0.5 & +3.8 \\
19 & Elimination & -0.6 & -0.4 & -0.6 & -1.4 & +0.9 \\
20 & Identification & +4.0 & +3.7 & +3.8 & +4.0 & +3.9 & +4.0 \\
\hline
\end{tabular}

Automated comparison information was found to be supportive of examiner conclusions, most notably the ACCF here. CMC had lower performance than the ACCF with Sets 17 and 18. Set 17 also proved to be challenging with the examiners due to relatively smooth breech face and firing pin impression areas of the Ruger LC9. In Set 18, the Taurus 24/7 G2 cartridge cases resulted in a small breech face area after pre-processing the surfaces due to trimming the flowback features. This led to less total comparison cells being used for comparison, and therefore may have resulted in less cells being labeled as congruent. However, the examiners performed well on this set. Further research into performance of these two firearms over many more cartridge cases and ammunition brands may provide some insight into these results. 
As previously mentioned, the LLRs displayed in Table 7 are generally indicative of the ground truth, with stronger support being produced for the ground truth elimination sets. Set 17 resulted in a LLR that is close to zero meaning it will be interpreted to provide very little support towards a ground truth identification. In difficult comparisons such as this, additional information can be useful. Looking at Set 17 in Table 8, the automated comparison information metrics actually reduced the LLR because the algorithms performed poorly on this comparison. The adaptive approach, however, resulted in an increase of 3.0 to the LLR when compared to using only the participant conclusions. Evidential value is now able to be provided for this comparison that supports the true proposition. Had only examiner conclusions been utilized based on categorical conclusions, an inconcluisve conclusion would likely be the result.

There are some limitations in this study, with the obvious being a small sample size of examiners. To implement a Bayesian network for practitioners to utilize for calculating LRs, not only would hundreds of examiners be needed, but comparison results from many different firearm models and different brands of ammunition would also be necessary. Different ammunition characteristics may further complicate the comparison process, although the flexibility of a Bayesian network allows for the accommodation of additional variables such as the ammunition. This will ensure robust performance.

\section{Conclusions}

The methods described in this paper detail the evaluation and application of a Bayesian network for calculating LRs from visual comparisons. With additional work, implementation would be feasible for use in routine casework. Furthermore, automated comparison information, along with any other necessary variables, are able to be easily combined to support visual comparison conclusions. These additional variables are especially useful in difficult comparisons, such as Set 17 from this study. Fifteen of 17 examiners selected an inconclusive conclusion for Set 17, but using additional information in the Bayesian network the LLR that resulted was 3.3. This LLR provides strong support for a same-source proposition, highlighting the benefits to this approach.

The double-casting process used in this study could provide some advantages to the forensic science community through training and proficiency testing. Creating training sets from cartridge cases identified through research to be difficult and lead to errors would assist in preventing similar errors in casework. These would include examples from this study from Set 16, where the false positive was made, and Set 17, the Ruger LC9 cartridge cases that led most examiners to make an inconclusive conclusion. Training sets known to include subclass characteristics could also be replicated. The Smith \& Wesson SD9VE in Set 11 is a good example. This firearm has a metal injection molded (MIM) firing pin, known to display subclass characteristics [41]. However, 
only five of the 17 participants noted that potential subclass characteristics were present on these cartridge cases. While no errors were made on Set 11, failure to acknowledge the potential for subclass characteristics is concerning. A proficiency test conducted by the European Network of Forensic Science Institutes (ENFSI) [42] using the Rivera cartridge cases [43] is also a good example of the effect of subclass characteristics. Multiple examiners made false positive errors due to prominent subclass characteristics present on the breech face impression areas of cartridge cases fired by Smith \& Wesson SW40VE pistols in that test. Double-casts of sets such as these should be prevalent in the comparison sets presented to firearm examiners during training, and similar sets should be utilized in proficiency tests.

\section{Acknowledgments}

The authors would like to sincerely thank all examiners that volunteered to participate in this study. The authors would like to acknowledge use of the supercomputing system Thorny Flat at WVU, which is funded in part by the National Science Foundation EPSCoR Research Infrastructure Improvement Cooperative Agreement \#1003907, National Science Foundation Major Research Instrumentation Program (MRI) Award \#1726534, the state of West Virginia (WVEPSCoR via the Higher Education Policy Commission) and WVU. The authors would also like to acknowledge the firearm and tool mark analysis team at the National Institute of Standards and Technology (NIST)

for providing access to their tool mark research software. The software was applied in this paper to calculate the ACCF and CMC comparison results.

\section{References}

[1] Stephen G. Bunch and Douglas P. Murphy. A comprehensive validity study for the forensic examination of cartridge cases. AFTE Journal, 35(2):201-203, 2003.

[2] Dennis J. Lyons. The identification of consecutively manufactured extractors. AFTE Journal, 41(3):246-256, 2009.

[3] Dawn LaPorte. An empirical and validation study of breechface marks on .380 ACP caliber cartridge cases fired from ten consecutively finished Hi-Point C9 pistols. AFTE Journal, 43(4):303-309, 2011.

[4] Thomas G. Fadul Jr, Gabriel A. Hernandez, Stephanie Stoiloff, and Sneh Gulati. An empirical study to improve the scientific foundation of forensic firearm and tool mark identification utilizing 10 consecutively manufactured slides. AFTE Journal, 45(4):376-393, 2013.

[5] Erich D. Smith. Cartridge case and bullet comparison validation study with firearms submitted in casework. AFTE Journal, 36(4):130-135, 2004. 
[6] David P. Baldwin, Stanley J. Bajic, Max Morris, and Daniel Zamzow. A study of false-positive and false-negative error rates in cartridge case comparisons. Retrieved from https://www.ncjrs.gov/pdffiles1/nij/249874.pdf, 2014.

[7] Angela Stroman. Empirically determined frequency of error in cartridge case examinations using a declared double-blind format. AFTE Journal, 46(2):157-175, 2014.

[8] James E. Hamby, Stephen Norris, and Nicholas D.K. Petraco. Evaluation of GLOCK 9 mm firing pin aperture shear mark individuality based on 1,632 different pistols by traditional pattern matching and IBIS pattern recognition. Journal of Forensic Sciences, 61(1):170-176, 2015.

[9] Tasha P. Smith, G. Andrew Smith, and Jeffrey B. Snipes. A validation study of bullet and cartridge case comparisons using samples representative of actual casework. Journal of Forensic Sciences, 61(4):939-946, 2016.

[10] James E. Hamby, David J. Brundage, Nicholas D. K. Petraco, and James W. Thorpe. A worldwide study of bullets fired from 10 consecutively rifled 9 MM RUGER pistol barrels_-analysis of examiner error rate. Journal of Forensic Sciences, 64(2):551-557, 2018.

[11] National Academy of Sciences. Strengthening Forensic Science in the United States: A Path Forward. The National Academies Press, 2009.

[12] President's Council of Advisors on Science and Technology. Forensic Science in Criminal Courts: Ensuring Scientific Validity of Feature-Comparison Methods, 2016.

[13] Pierre Duez, Todd Weller, Marcus Brubaker, Richard E. Hockensmith, and Ryan Lilien. Development and validation of a virtual examination tool for firearm forensics. Journal of Forensic Sciences, 63(4):1069-1084, 2018.

[14] Erwin J.A.T. Mattijssen, Cilia L.M. Witteman, Charles E.H. Berger, Nicolaas W. Brand, and Reinoud D. Stoel. Validity and reliability of forensic firearm examiners. Forensic Science International, 307, 2020.

[15] INTERPOL. INTERPOL Ballistics Information Network: Handbook on the Collection and Sharing of Ballistics Data, 3rd edition, 2014.

[16] Eric Law and Keith Morris. The utility of double-casting for creating cartridge case reproductions. AFTE Journal, 52(1):26-39, 2020.

[17] Eric F. Law and Keith B. Morris. Three-dimensional analysis of cartridge case double-casts. Journal of Forensic Sciences, September 2020.

[18] Silvia Salini and Ron S. Kenett. Bayesian networks of customer satisfaction survey data. Journal of Applied Statistics, 36(11):1177-1189, 2009.

[19] Paul Arora, Devon Boyne, Justin J. Slater, Alind Gupta, Darren R. Brenner, and Marek J. Druzdzel. Bayesian networks for risk prediction using real-world data: A tool for precision medicine. Value in Health, 22(4):439-445, 2019. 
[20] Franco Taroni, Alex Biedermann, Silvia Bozza, Paolo Garbolino, and Colin Aitken. Bayesian networks for probabilistic inference and decision analysis in forensic science. Wiley, Chichester, England, 2014.

[21] Cadre Forensics. X3P Viewer Software. Available by request at www.cadreforensics.com as of $21-F e b-2020$.

[22] AFTE. AFTE criteria for identification committee final report. AFTE Journal, 24(3), 1992.

[23] Qualtrics. https://www.qualtrics.com. Last accessed 21-Feb-2020.

[24] Open Forensic Metrology Consortium. OpenFMC \& X3P. Available at https://www.cadreforensics.com/x3p.html as of August 21, 2019.

[25] Itiel E. Dror, William C. Thompson, Christian A. Meissner, Irv Kornfield, Dan Krane, Michael Saks, and Michael Risinger. Letter to the editor- context management toolbox: A linear sequential unmasking (LSU) approach for minimizing cognitive bias in forensic decision making. Journal of Forensic Sciences, 60(4):1111-1112, 2015.

[26] John Song. Proposed NIST Ballistics Identification System (NBIS) based on 3D topography measurements on correlation cells. AFTE Journal, 45(2):184-194, 2013.

[27] W. Chu, M. Tong, and J. Song. Validation tests for the congruent matching cells (CMC) method using cartridge cases fired with consecutively manufactured pistol slides. AFTE Journal, 45(4):361-366, 2013.

[28] John Song, Theodore V. Vorburger, Wei Chu, James Yen, Johannes A. Soons, Daniel B. Ott, and Nien Fan Zhang. Estimating error rates for firearm evidence identifications in forensic science. Forensic Science International, 284:15-32, 32018.

[29] Norsys Software Corp. Netica Application. https://www.norsys.com/netica.html, 2020.

[30] Russell Almond. RNetica: R interface to Netica $(R)$ Bayesian Network Engine. R package version 0.7-3.

[31] Uffe B. Kjaerulff and Anders L. Madsen. Bayesian Networks and Influence Diagrams: A Guide to Construction and Analysis. Springer-Verlag GmbH, 2012.

[32] Fei Zheng and Geoffrey I. Webb. Encyclopedia of Machine Learning, chapter Tree Augmented Naive Bayes, pages 990-991. Springer US, Boston, MA, 2010.

[33] Fei Zheng and Geoffrey I. Webb. Encyclopedia of Machine Learning, chapter Semi-Naive Bayesian Learning, pages 889-892. Springer US, Boston, MA, 2010.

[34] Kevin B. Korb. Encyclopedia of Machine Learning, chapter Learning Graphical Models, pages 584-590. Springer US, Boston, MA, 2010.

[35] GeNIe. BayesFusion, LLC, 2020. Available at https://www.bayesfusion.com. 
[36] Judea Pearl. Probabilistic Reasoning in Intelligent Systems: Networks of Plausible Inference. Morgan Kaufmann Series in Representation and Reasoning, 1988.

[37] Tom Fawcett. ROC graphs: Notes and practical considerations for data mining researchers. Intelligent Enterprise Technologies Laboratory. HP Laboratories Palo Alto, 2003.

[38] Peter A. Flach. Encyclopedia of Machine Learning, chapter ROC Analysis, pages 869-875. Springer US, Boston, MA, 2010.

[39] Xavier Robin, Natacha Turck, Alexandre Hainard, Natalia Tiberti, Frdrique Lisacek, Jean-Charles Sanchez, and Markus Mller. pROC: an open-source package for R and S+ to analyze and compare ROC curves. BMC Bioinformatics, 12:77, 2011.

[40] U.S. Department of Health and Human Services, Food and Drug Administration, Office of the Commissioner (OC), Good Clinical Practice Program (GCPP). Guidance for Sponsors, Clinical Investigators, and IRBs. Data Retention When Subjects Withdraw from FDA-Regulated Clinical Trials, 2008.

[41] Ronald Nichols. Subclass characteristics: from origin to evaluation. AFTE Journal, 50(2), 2018.

[42] Petra Pauw-Vugts, Alice Walters, Leif Øren, Kripos, and Leopold Pfoser. Faid2009: Proficiency test and workshop. AFTE Journal, 45(2):115-127, 2013.

[43] Gene C. Rivera. Subclass characteristics in Smith \& Wesson SW40VE Sigma pistols. AFTE Journal, 39(3):253-258, 2007. 


\section{Concluding remarks}

Double-casting has been shown in this study to produce accurate reproductions with little concern for significant variability within and between molds of the same master cartridge case. This was confirmed based on visual examination, 2D automated comparisons, and 3D automated comparisons. Reproductions are able to be made for use as training and testing sets. These double-casts could then be distributed to any interested parties, ensuring that all individuals receive items with the same level of detail.

For use as training sets, cartridge cases found through research and known to be difficult should be replicated. These would compose the best possible comparison sets for trainees. An additional benefit is that all trainees in a class would be examining the same sets at the same time and can discuss what is observed. From this study these would include the cases from Set 16 where the false positive was made. This is a set that trainees should be aware of so that the same mistakes are not repeated. Set 17 should also be included due to the difficulty of that set. This may be an instance where trainees could learn when inconclusive conclusions are acceptable responses, and that an identification should not be made due to lack of reproducible features.

Double-casts also have similar use for proficiency testing. If all participants in a proficiency test are provided with different cartridge cases, then a failure may indicate that a particular test was more difficult. This is not necessarily a fair way to test proficiency. Using double-casts, all participants would have samples with the same level of detail. In this scenario if there was a failure, the responses would be directly comparable to the others that took the test. A failure is more meaningful in this instance because proficiency is being directly compared between all examiners. Combined with double-casts for training, this would help to achieve some standardization in the firearms examination discipline.

Examiner conclusion variability was analyzed in this study using cartridge case double-casts. A practicing firearm examiner assessed the master cartridge cases that were selected for the test sets to ensure a range of difficulty was represented. The master cartridge cases were then replicated through the double-casting process. The reproductions were sent to examiners for comparison. Their conclusions were compared across each set because they all received double-casts with the same level of detail. 
Significant variability between examiner conclusions in this study was found. Ground truth elimination comparisons resulted in greater conclusion variability than the ground truth identification comparisons. This was indicated by four of the seven ground truth identification sets resulting in all examiners coming to the correct ground truth conclusion. No ground truth elimination sets resulted in every participant coming to an elimination conclusion. It is important to note that only one false positive was made and there were zero false negatives, meaning the observed variability was contained almost completely within the inconclusive categories. Although the variability was mainly due to inconclusives, it is still concerning in terms of missed identifications and eliminations. On sets where there is consensus for an elimination conclusion, and only an elimination conclusion, then examiners should be making the elimination instead of coming to an inconclusive which provides no evidential value.

While less inconclusive conclusions and more correct ground truth conclusions are desired, there was some hesitancy to come to definitive conclusions without the firearm present. Some participants acknowledged the ability to examine the firearm for each set and potentially conduct additional test fires may have increased confidence in a definitive conclusion leading to less total inconclusive conclusions. This is a limitation of this study and other similar studies in terms of the realistic nature of the comparison process when compared to casework.

The ground truth elimination sets all contained questioned cartridge cases with the same class characteristics as the known cartridge cases. For 12 of the 13 elimination sets the questioned cartridge case was from the same model firearm, and for the remaining elimination set the firearm was a different model but still showed the same class characteristics (Set 7). These led to the elimination comparisons being close non-matches and were designed in this way to present challenging scenarios to the examiners. There was a single false positive on Set 16, although thirteen of the remaining 16 participants all made the correct elimination. It is unknown why this error was made by an examiner with over ten years of experience in firearms examination. However, this participant also made nine inconclusive A conclusions on ground truth eliminations. This participant was eager to indicate the degree of similarity across these sets, ignoring the differences. While all ground truth elimination sets were close non-matches, this was unexpected and is a concerning result.

Another concerning observation from this study is that some agency policies do not allow examiners to eliminate based on individual characteristics. In terms of this study, that means all elimination comparisons could, at best, result in inconclusive $\mathrm{C}$ conclusions because all of the class characteristics were similar. The participants in this study that have a policy stated as such changed their conclusions from inconclusives to eliminations for all relevant comparisons when provided with the opportunity. Examiners should not be restricted in their conclusions in such a way that reduces the evidential value of comparisons when the examiners may observe sufficient 
dissimilarities and be confident in making an elimination conclusion. The fact that an identification can be made on individual characteristics but an elimination cannot is illogical.

A Bayesian network was applied to the examiner conclusion data. LRs were calculated based on the examiner conclusions and were found to be informative towards the ground truth. However, the main benefit to an approach such as this is in accounting for difficult comparisons. The best example of this is using Set 17. Fifteen of 17 examiners made an inconclusive conclusion on this set. Furthermore, the automated comparison methods performed poorly on this set due to the lack of reproducible markings left by this particular Ruger LC9. By adding additional information into the LR calculation through use of the Bayesian network, such as the reproducibility of markings left by the firearm, an LR supporting an identification resulted. This method produces much more useful information from the comparison than simply stating it was inconclusive.

While the LRs produced from the Bayesian network created in this study are based on the participating examiners and the specific comparisons provided to compose the test sets, it would be feasible to incorporate much larger datasets into the network. This would encompass additional examiners, firearms, and ammunition types which would make the LRs produced using the network more robust to casework scenarios. The network could then be used to provide LRs to support examiners in their conclusions.

Automated comparison information was found to be supportive of examiner conclusions. There were sets where the ACCF and $\mathrm{CMC}$ data were indicative of the ground truth through use of students analyzing the data without seeing the physical cartridge cases or images of the impression areas. There were also sets where the automated comparison result interpretation produced errors while the examiners comparing the cartridge cases performed well. This indicates that a combination of examiner conclusions and automated comparison data may lead to stronger conclusions than either in isolation. However, interpretation of the automated comparison results by experienced examiners, in conjunction with visual comparisons, needs further study. In the Bayesian network, the ACCF was found to be the more informative metric. While both the ACCF and CMC metrics performed poorly on Set 17, the CMC metric also performed poorly on Set 18. Further research is needed to identify the causes of low performance on these two firearms.

Areas for further research were identified through this study. Two topics listed above include further evaluation of poor performing firearms using the ACCF and CMC metrics, as well as interpretation of automated comparison results by experienced examiners. Additional error rate studies should continue to be conducted using a variety of different firearms and ammunition. This will help to produce a robust estimate of error in cartridge case comparisons. A compilation of this data into a Bayesian network would allow for routine LR calculations in casework based on examiner conclusions, automated comparison results, and any additional relevant information. Error rate studies should consider use of virtual comparison microscopy (VCM) as 3D imaging systems 
are becoming more common in the forensic science community. VCM may also allow for further analysis of errors because specific areas of similarities and differences can be annotated. This may provide more insight into false positive, false negative, and inconclusive conclusions. 


\section{Supplementary material}

Included below are images of each test set sent to participating examiners. All 3D scans were acquired using a Sensofar ${ }^{\circledR} S$ neox. The $2 \mathrm{D}$ images that are displayed were saved using the Cadre Forensics X3P Viewer software (available by request at https://www. cadreforensics. com/Software.html). 


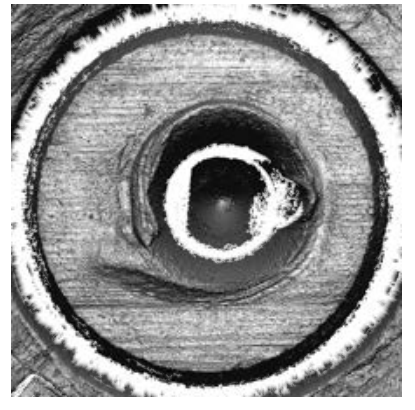

Q (GB)

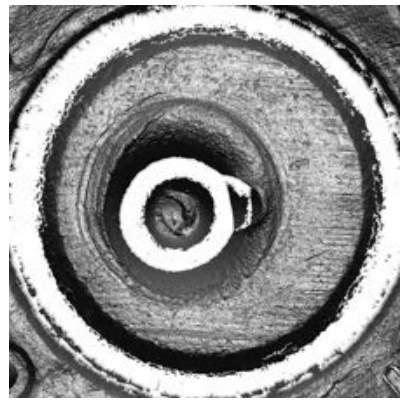

K1 (AY)

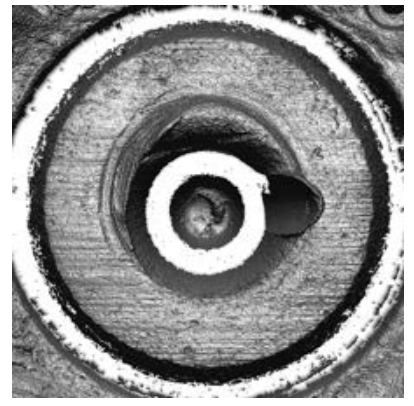

K2 (YB)

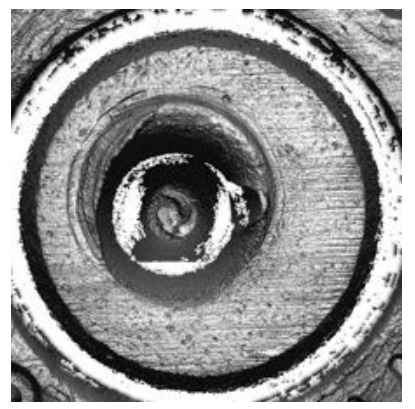

K3 (AG)

Set 01: Taurus 24/7 G2, Elimination

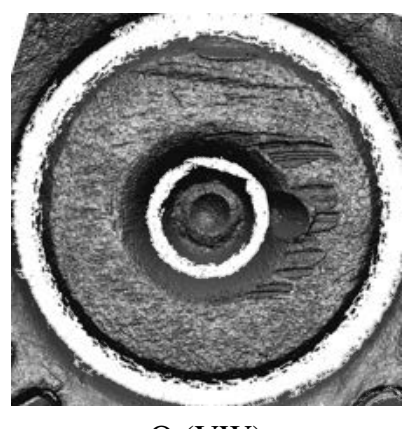

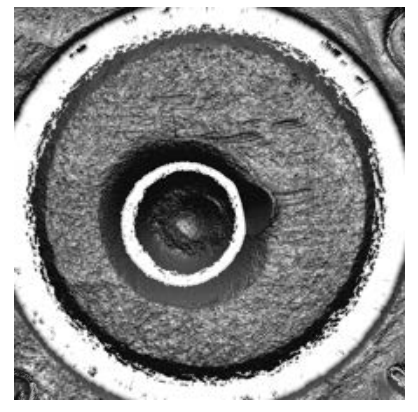

K1 (ZZ)

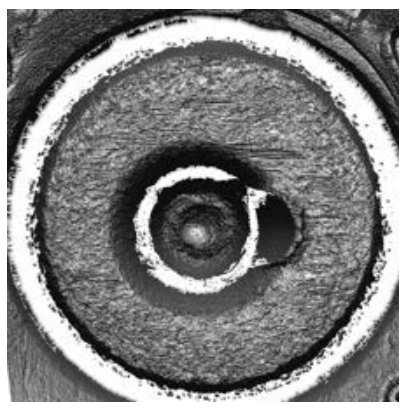

K2 (GS)

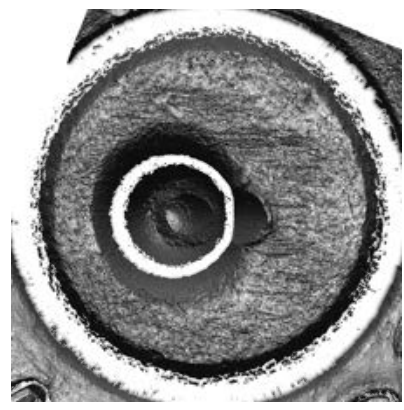

K3 (MO)

Set 02: SCCY CPX-2, Elimination

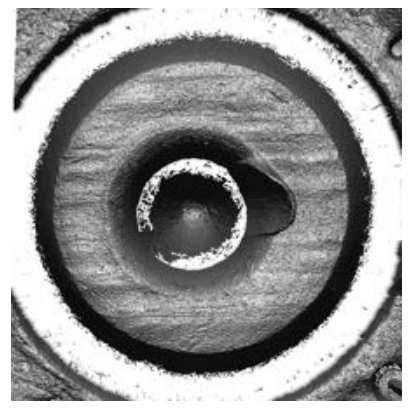

Q (FY)

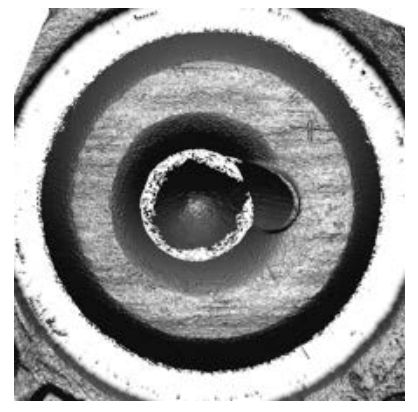

K1 (VF)

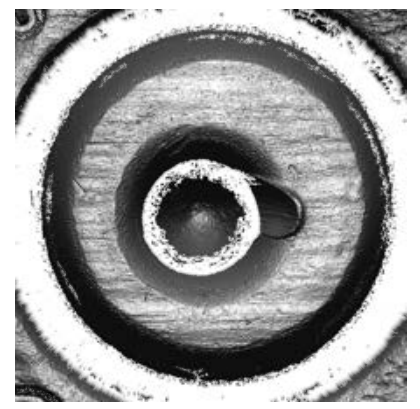

K2 (GM)

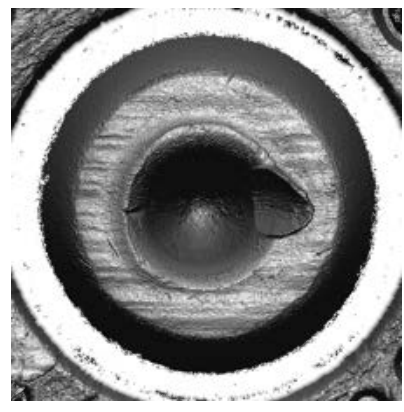

K3 (VI)

Set 03: Springfield XD9, Identification 


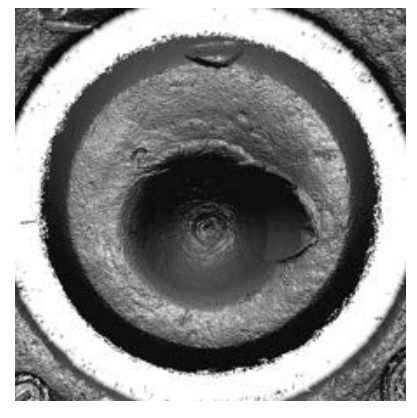

Q (UV)

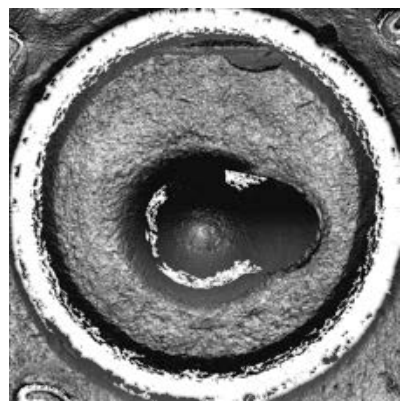

K1 (FF)

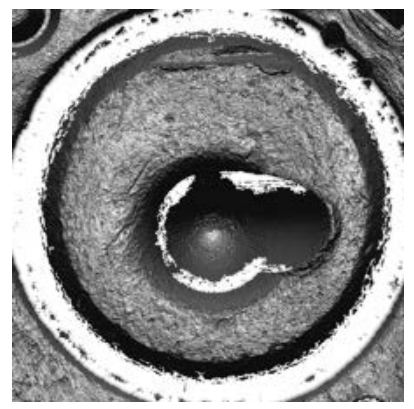

K2 (NT)

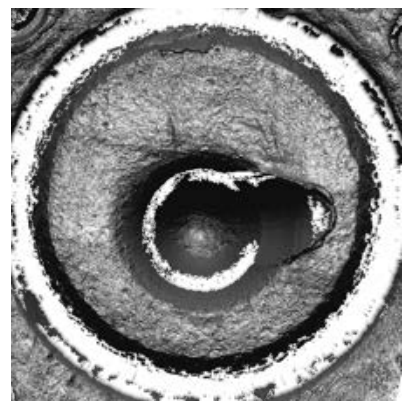

K3 (JA)

Set 04: Ruger LC9, Elimination

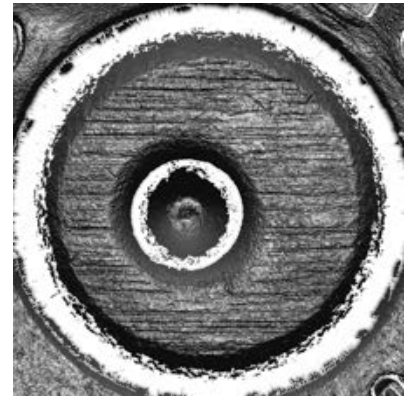

$\mathrm{Q}$ (SA)

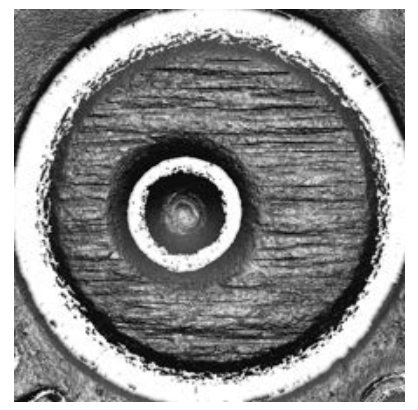

K1 (XU)

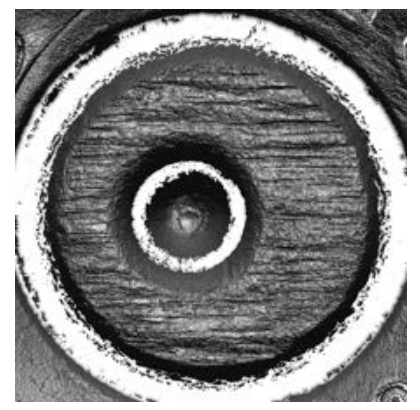

$\mathrm{K} 2(\mathrm{KX})$

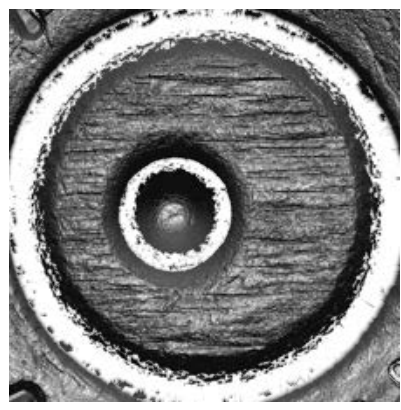

K3 (BX)

Set 05: Hi-Point C9, Elimination

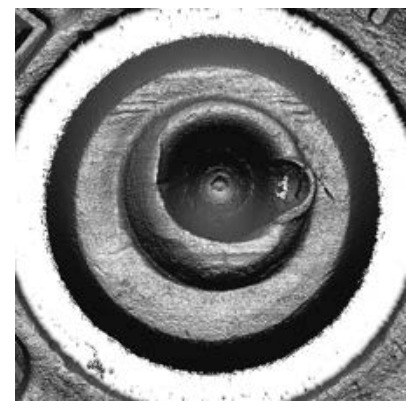

$\mathrm{Q}(\mathrm{PO})$

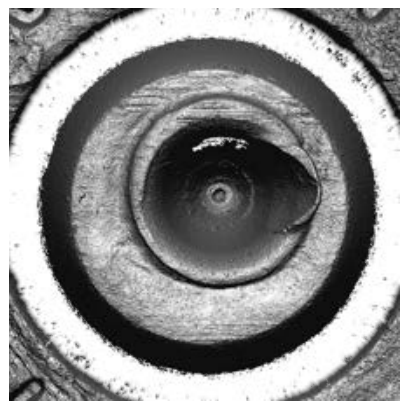

K1 (GO)

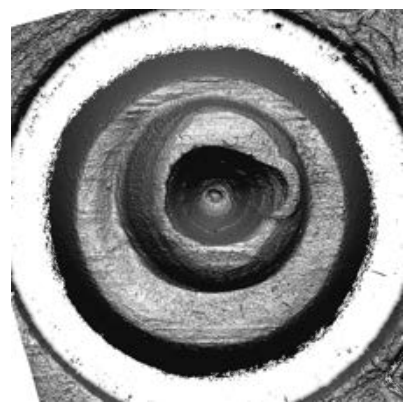

K2 (PD)

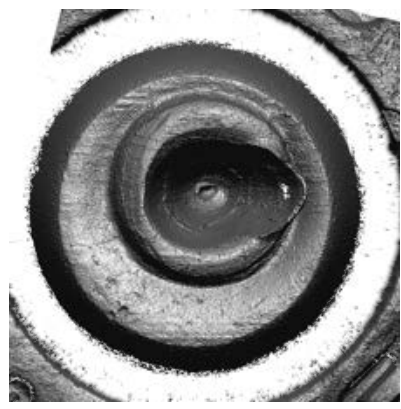

K3 (OX)

Set 06: Keltec PF9, Identification 


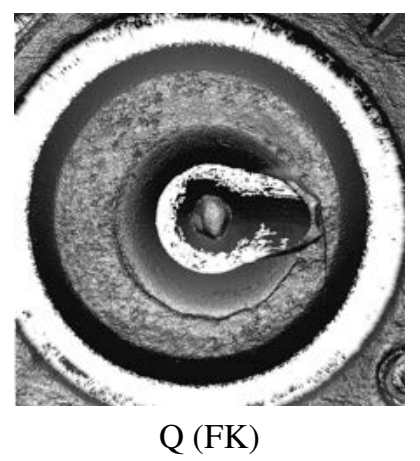

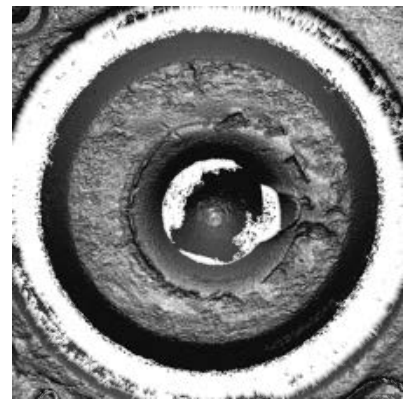

K1 (JN)

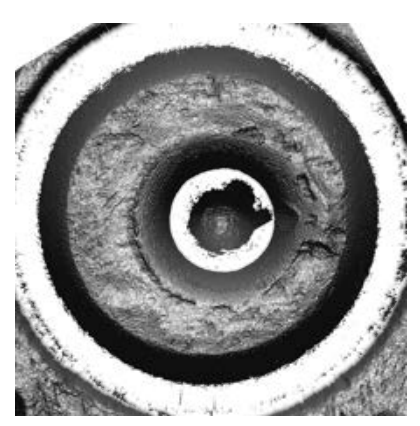

K2 (KR)

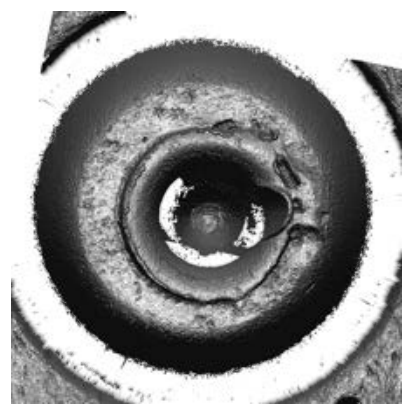

K3 (QX)

Set 07: Ruger P95, Elimination

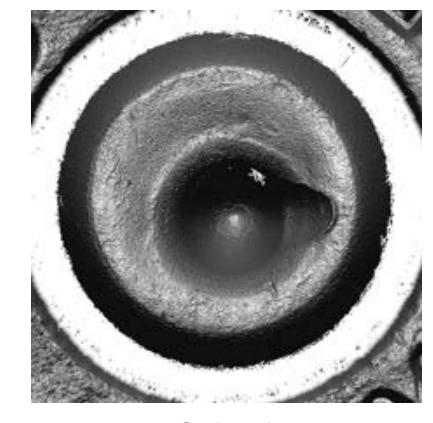

Q (NR)

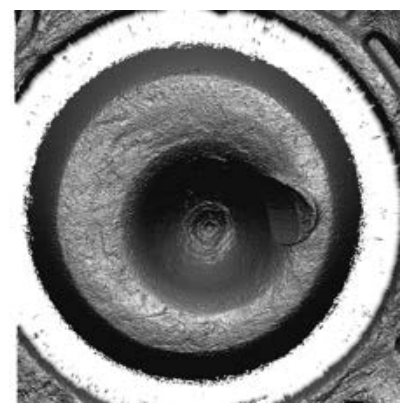

K1 (JP)

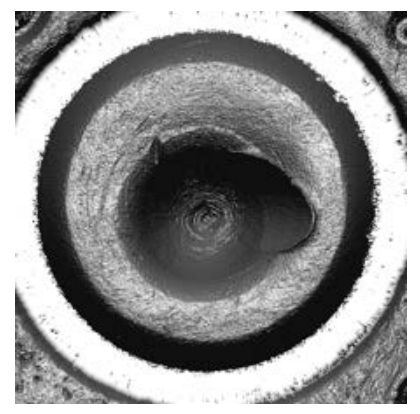

K2 (QF)

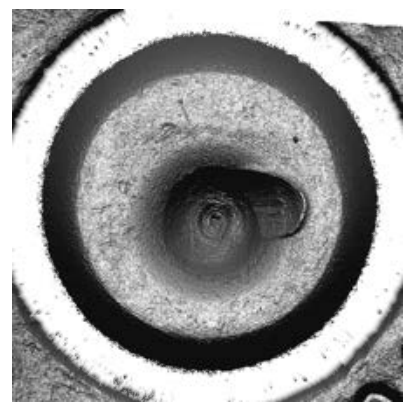

K3 (PX)

Set 08: Ruger LC9, Elimination

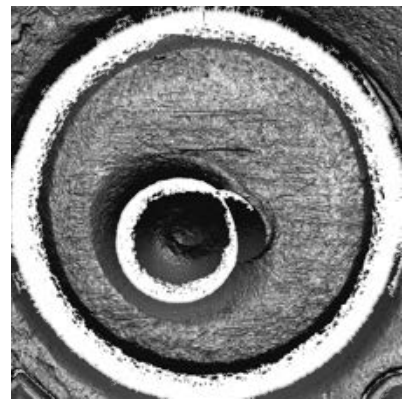

$\mathrm{Q}(\mathrm{CM})$

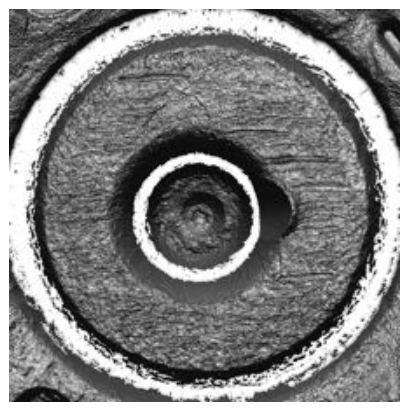

K1 (CJ)

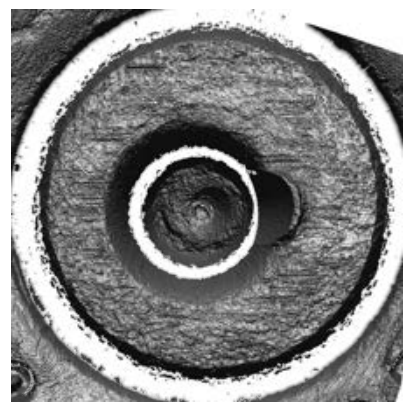

K2 (DV)

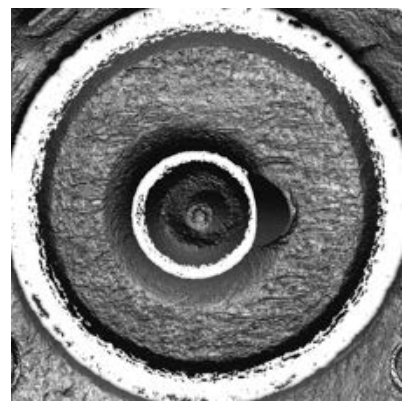

K3 (QB)

Set 09: SCCY CPX-2, Elimination 


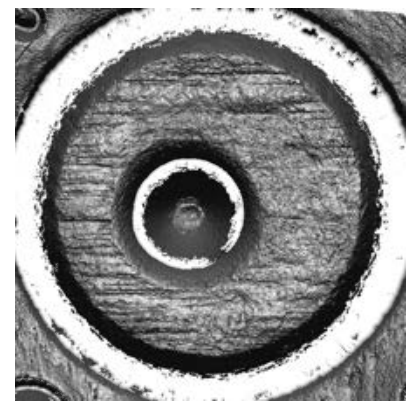

$\mathrm{Q}$ (SK)

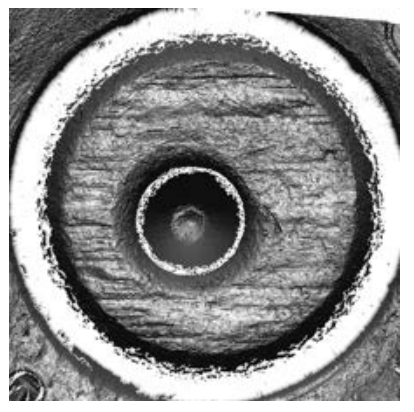

K1 (BV)

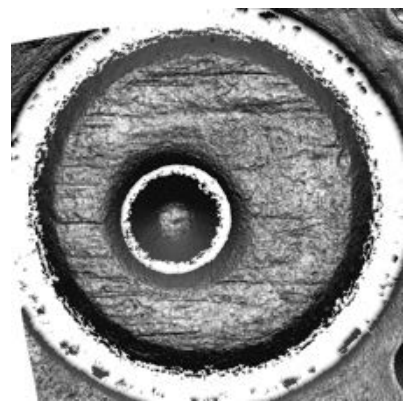

$\mathrm{K} 2(\mathrm{HH})$

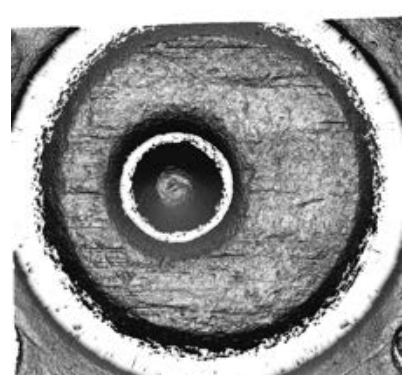

K3 (NM)

Set 10: Hi-Point C9, Identification

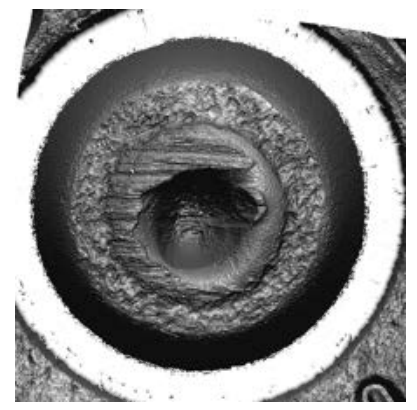

$\mathrm{Q}(\mathrm{BR})$

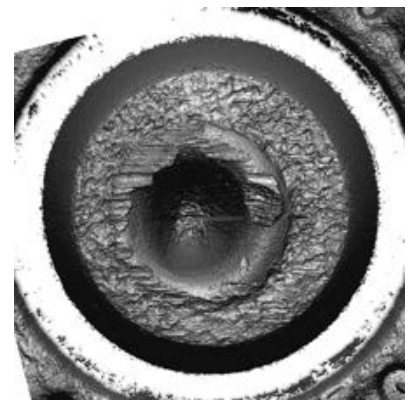

K1 (YP)

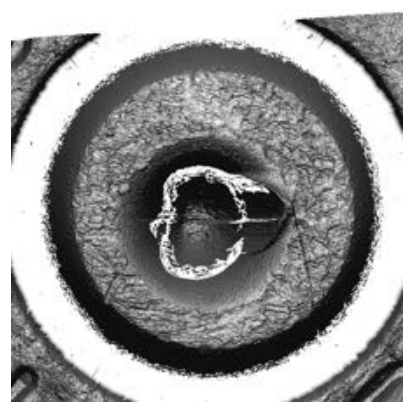

K2 (LO)

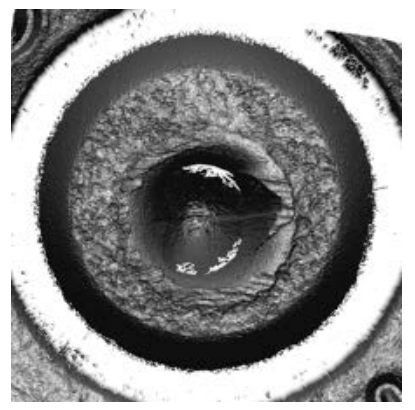

K3 (KK)

Set 11: Smith \& Wesson SD9VE, Identification
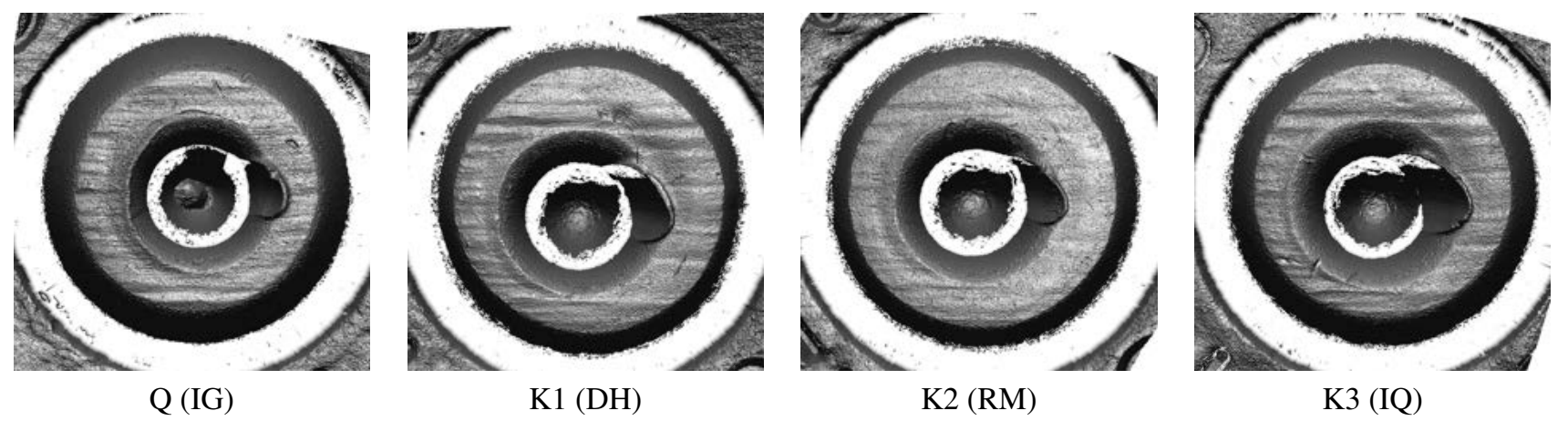

Set 12: Springfield XD9, Elimination 


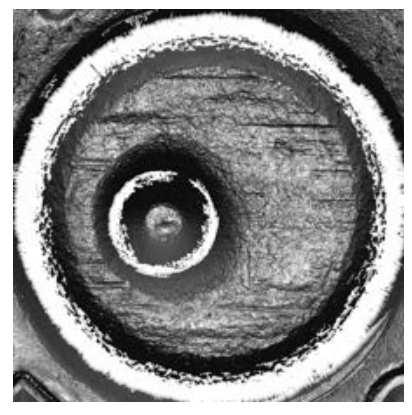

Q (GL)

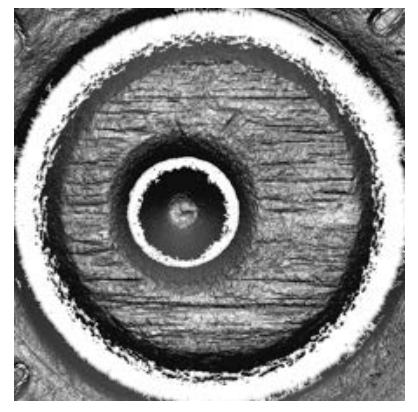

$\mathrm{K} 1$ (AC)

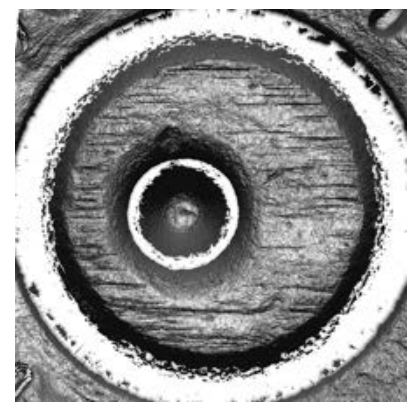

K2 (TE)

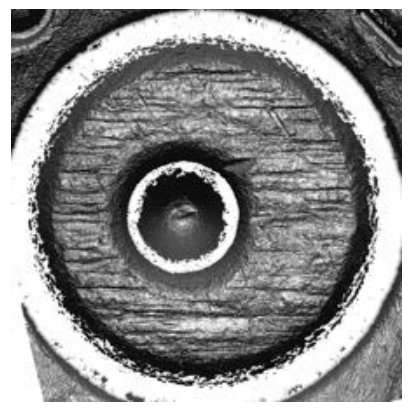

K3 (XW)

Set 13: Hi-Point C9, Elimination

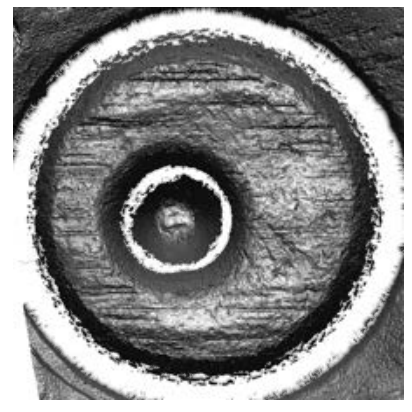

Q (KY)

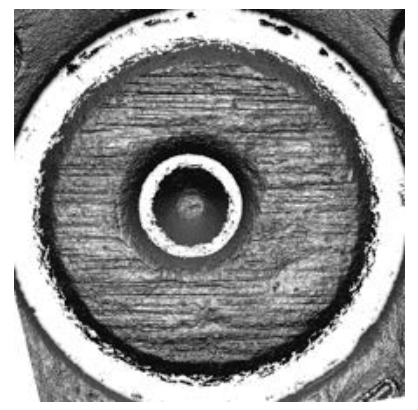

K1 (VR)

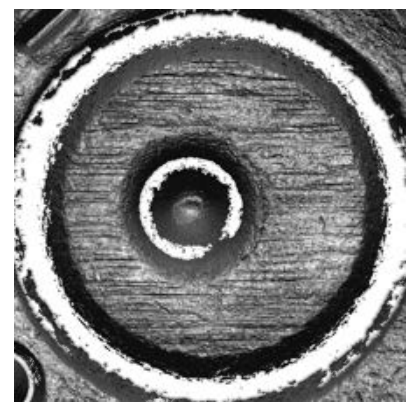

K2 (YM)

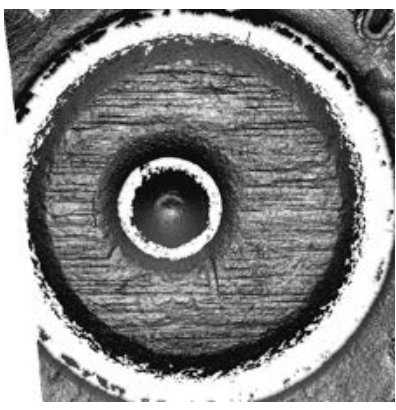

K3 (PR)

Set 14: Hi-Point C9, Elimination

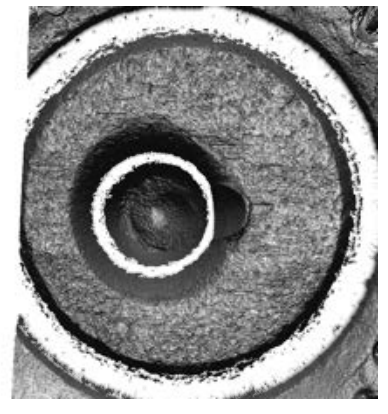

Q (LZ)

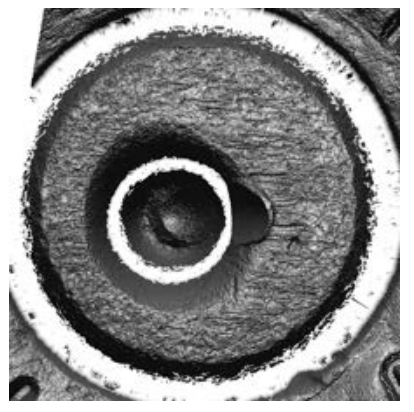

K1 (LY)

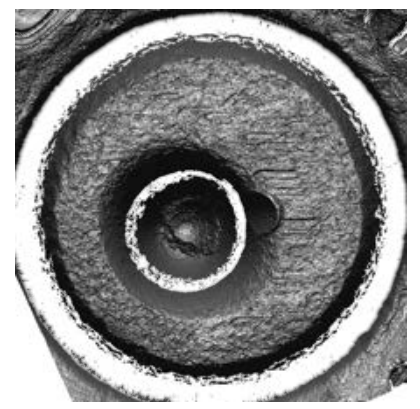

K2 (UF)

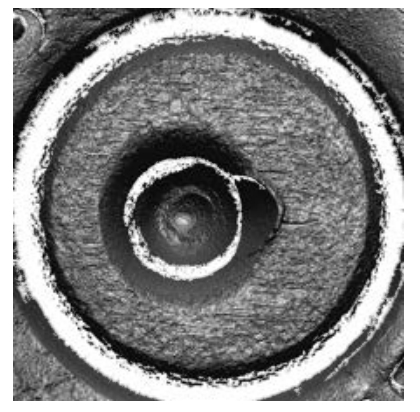

$\mathrm{K} 3(\mathrm{MN})$

Set 15: SCCY CPX-2, Elimination 


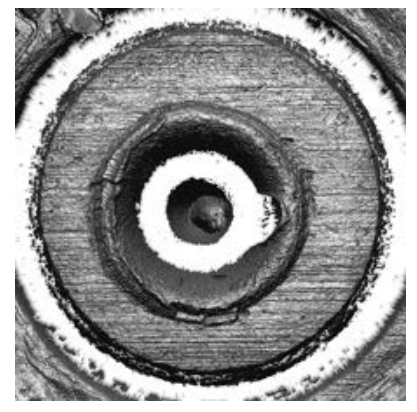

Q (RG)

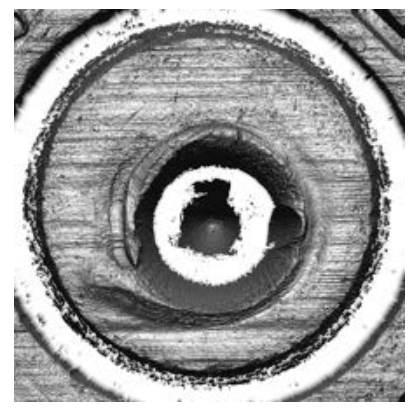

K1 (RD)

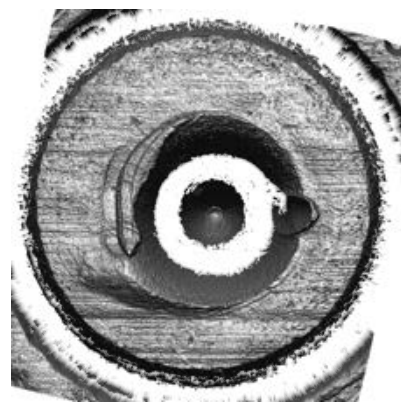

$\mathrm{K} 2$ (OC)

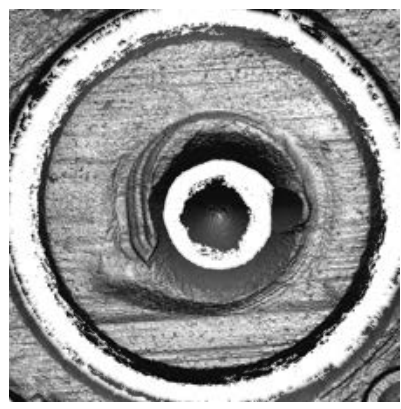

K3 (AW)

Set 16: Taurus 24/7 G2, Elimination

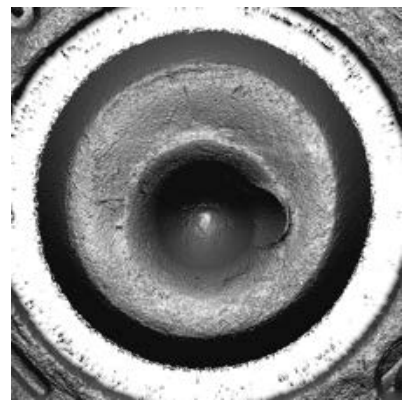

$\mathrm{Q}(\mathrm{YO})$

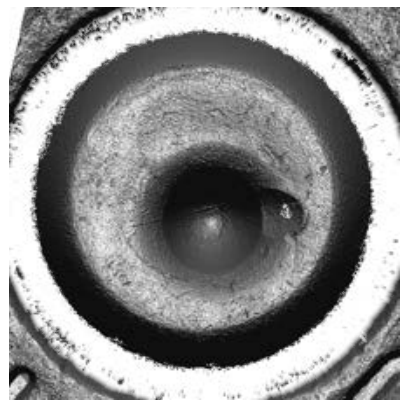

$\mathrm{K} 1$ (EI)

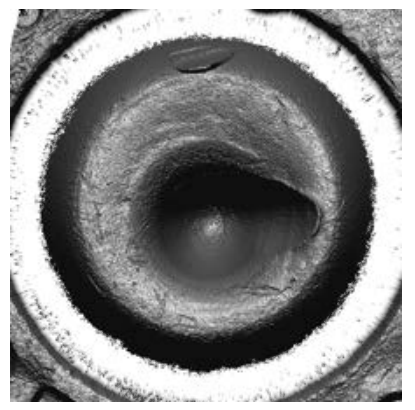

K2 (NS)

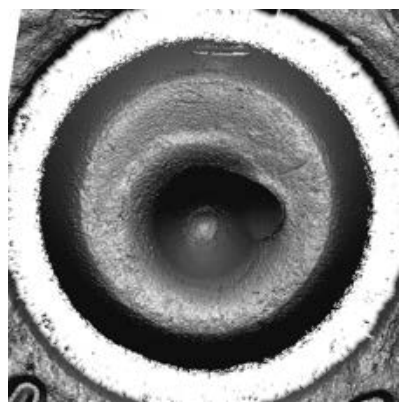

K3 (PT)

Set 17: Ruger LC9, Identification

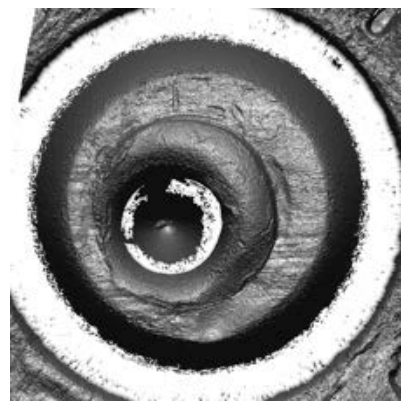

Q (ZT)

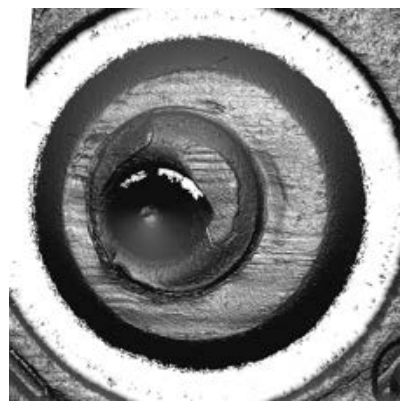

K1 (GE)

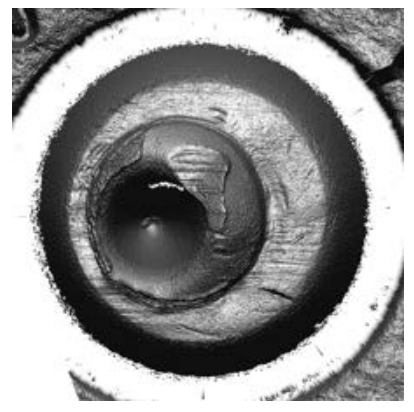

$\mathrm{K} 2(\mathrm{AJ})$

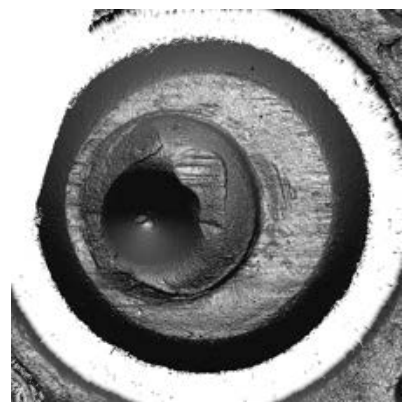

K3 (JT)

Set 18: Taurus 24/7 G2, Identification 

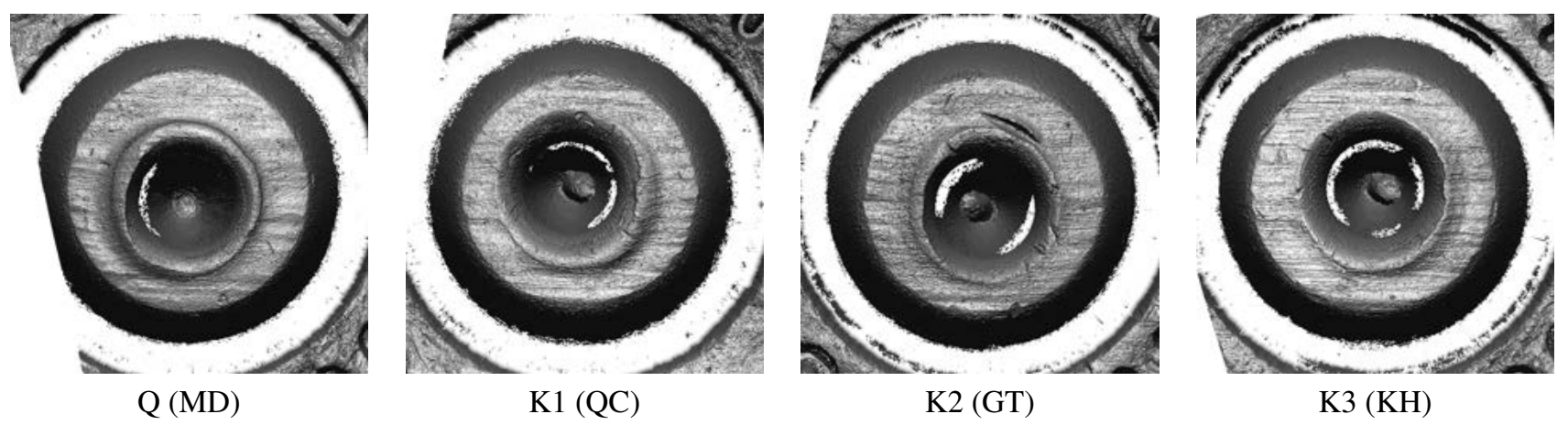

Set 19: Springfield XD9, Elimination

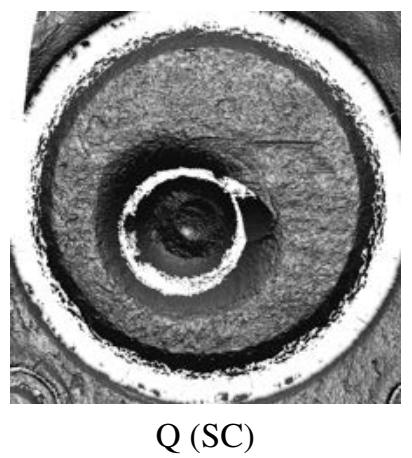

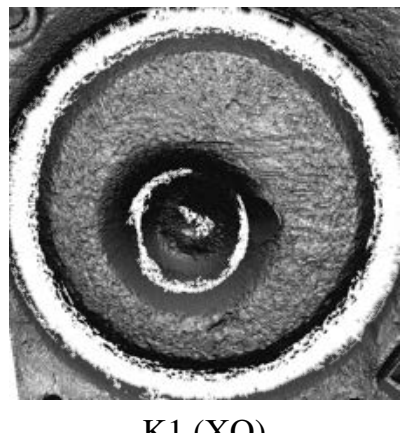

K1 (XO)

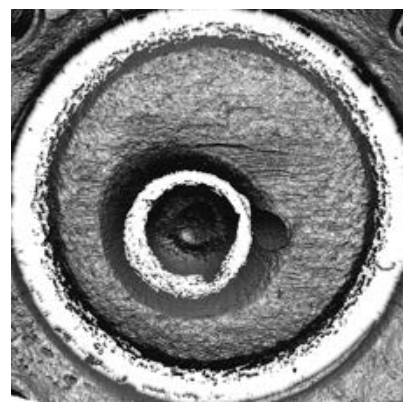

K2 (DU)

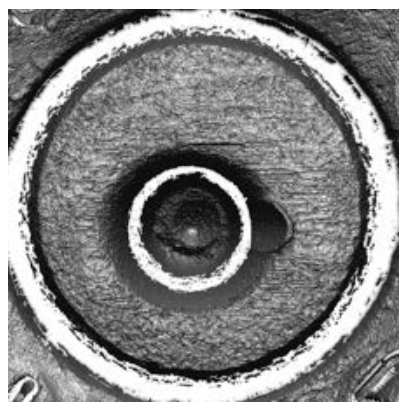

K3 (BJ)

Set 20: SCCY CPX-2, Identification 


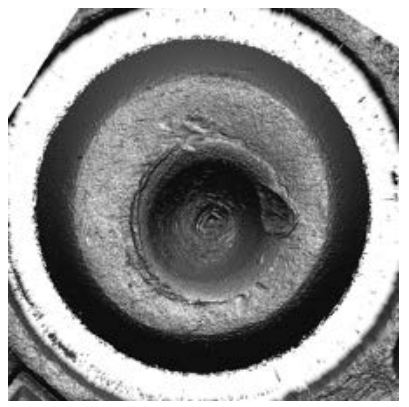

Firearm 1 - Ruger LC9 (LI)

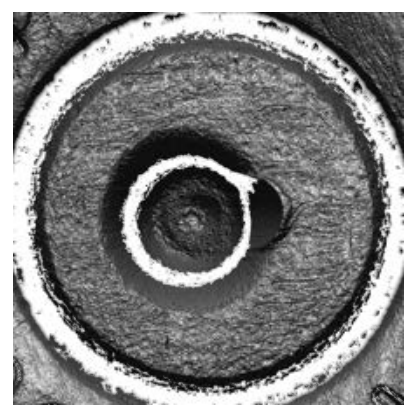

Firearm 3 - SCCY CPX-2

(AQ)

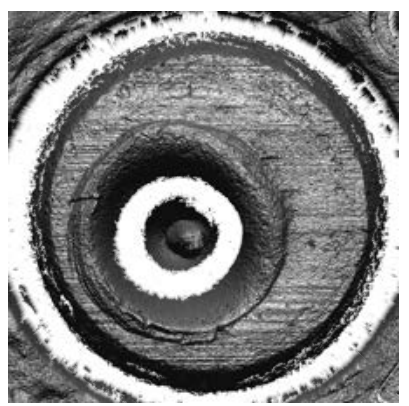

Firearm 5 - Taurus 24/7 G2 (NB)

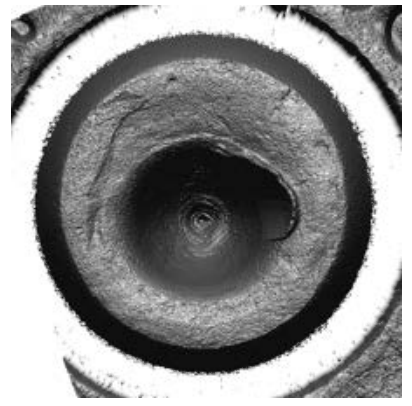

Firearm 1 - Ruger LC9

(MX)

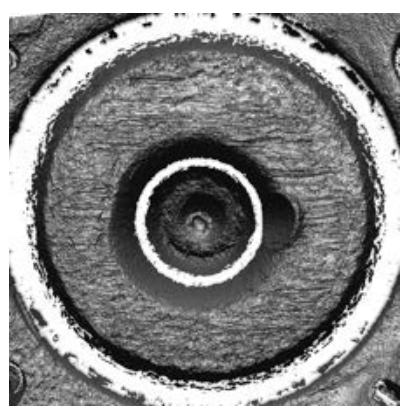

Firearm 3 - SCCY CPX-2

(KB)

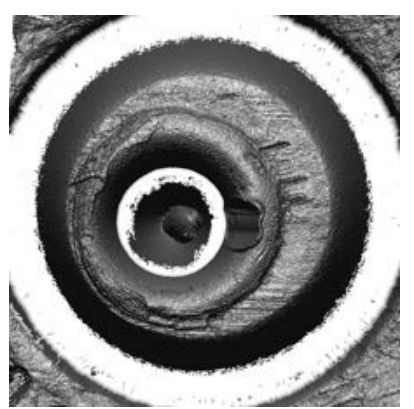

Firearm 5 - Taurus 24/7 G2 (XS)

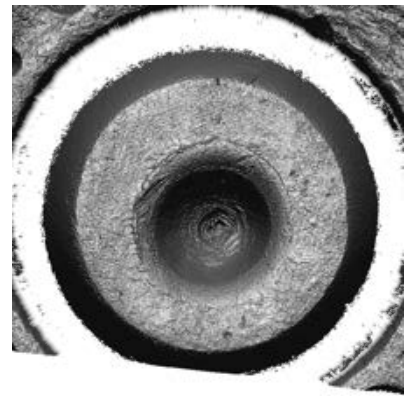

Firearm 1 - Ruger LC9

(UB)

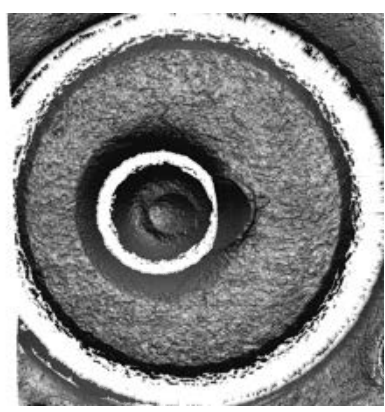

Firearm 4 - SCCY CPX-2

(TY)

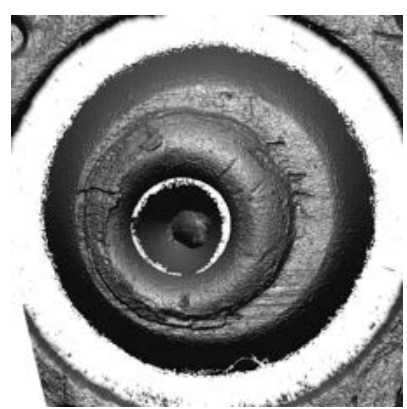

Firearm 5 - Taurus 24/7 G2

(RT)

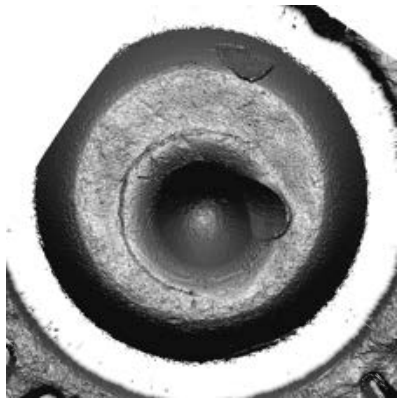

Firearm 2 - Ruger LC9 (DD)

Set 21: ten cartridge cases fired from five total firearms 